\title{
Front Matter: Volume 9147
}

, "Front Matter: Volume 9147," Proc. SPIE 9147, Ground-based and Airborne Instrumentation for Astronomy V, 914701 (28 July 2014); doi:

10.1117/12.2074593

SPIE Event: SPIE Astronomical Telescopes + Instrumentation, 2014, Montréal, Quebec, Canada 


\title{
Ground-based and Airborne Instrumentation for Astronomy V
}

\author{
Suzanne K. Ramsay \\ Ian S. McLean \\ Hideki Takami \\ Editors
}

22-26 June 2014

Montréal, Canada

Sponsored by

SPIE

Cooperating Organizations

American Astronomical Society (United States) • Australian Astronomical Observatory

(Australia) - Association of Universities for Research in Astronomy (AURA) • Canadian Astronomical Society (CASCA) (Canada) - Canadian Space Agency (Canada) • European Astronomical Society (Switzerland) • European Southern Observatory (Germany) • National Radio Astronomy Observatory • Royal Astronomical Society (United Kingdom) • Science \& Technology Facilities Council (United Kingdom)

Published by

SPIE 
The papers included in this volume were part of the technical conference cited on the cover and title page. Papers were selected and subject to review by the editors and conference program committee. Some conference presentations may not be available for publication. The papers published in these proceedings reflect the work and thoughts of the authors and are published herein as submitted. The publisher is not responsible for the validity of the information or for any outcomes resulting from reliance thereon.

Please use the following format to cite material from this book:

Author(s), "Title of Paper," in Ground-based and Airborne Instrumentation for Astronomy V, edited by Suzanne K. Ramsay, Ian S. McLean, Hideki Takami, Proceedings of SPIE Vol.9147 (SPIE, Bellingham, WA, 2014) Article CID Number.

ISSN: 0277-786X

ISBN: 9780819496157

Published by

SPIE

P.O. Box 10, Bellingham, Washington 98227-0010 USA

Telephone +1 3606763290 (Pacific Time) · Fax +1 3606471445

SPIE.org

Copyright @ 2014, Society of Photo-Optical Instrumentation Engineers.

Copying of material in this book for internal or personal use, or for the internal or personal use of specific clients, beyond the fair use provisions granted by the U.S. Copyright Law is authorized by SPIE subject to payment of copying fees. The Transactional Reporting Service base fee for this volume is $\$ 18.00$ per article (or portion thereof), which should be paid directly to the Copyright Clearance Center (CCC), 222 Rosewood Drive, Danvers, MA 01923. Payment may also be made electronically through CCC Online at copyright.com. Other copying for republication, resale, advertising or promotion, or any form of systematic or multiple reproduction of any material in this book is prohibited except with permission in writing from the publisher. The CCC fee code is 0277-786X/14/\$18.00.

Printed in the United States of America.

Publication of record for individual papers is online in the SPIE Digital Library.

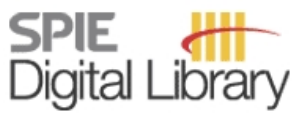

SPIEDigitalLibrary.org

Paper Numbering: Proceedings of SPIE follow an e-First publication model, with papers published first online and then in print and on CD-ROM. Papers are published as they are submitted and meet publication criteria. A unique, consistent, permanent citation identifier (CID) number is assigned to each article at the time of the first publication. Utilization of CIDs allows articles to be fully citable as soon as they are published online, and connects the same identifier to all online, print, and electronic versions of the publication. SPIE uses a six-digit CID article numbering system in which:

- The first four digits correspond to the SPIE volume number.

- The last two digits indicate publication order within the volume using a Base 36 numbering system employing both numerals and letters. These two-number sets start with 00, 01, 02, 03, 04, $05,06,07,08,09,0 A, O B \ldots$ OZ, followed by $10-1 Z, 20-2 Z$, etc.

The CID Number appears on each page of the manuscript. The complete citation is used on the first page, and an abbreviated version on subsequent pages. Numbers in the index correspond to the last two digits of the six-digit CID Number. 


\section{Contents}

\section{Part One}

Ixiii Conference Committee

Ixv Introduction

SESSION $1 \quad$ INSTRUMENT PROGRAMS AT MAJOR OBSERVATORIES

914702 Instrumentation at Gemini Observatory (Invited Paper) [9147-1]

S. J. Kleinman, M. Boccas, S. J. Goodsell, P. Gomez, R. Murowinski, A.-N. Chené,

D. Henderson, Gemini Observatory (United States)

914703 New developments in instrumentation at the W. M. Keck Observatory (Invited Paper) [9147-2]

S. M. Adkins, T. E. Armandroff, W. M. Keck Observatory (United States); M. P. Fitzgerald, Univ. of California, Los Angeles (United States); J. Johnson, W. M. Keck Observatory (United States); J. E. Larkin, Univ. of California, Los Angeles (United States); H. A. Lewis, W. M. Keck Observatory (United States); C. Martin, California Institute of Technology (United States); K. Y. Matthews, Univ. of California, Los Angeles (United States); J. X. Prochaska, Univ. of California, Santa Cruz (United States); P. Wizinowich, W. M. Keck Observatory (United States)

914704 Paranal instrumentation programme (Invited Paper) [9147-3]

L. Pasquini, M. Casali, A. Russel, European Southern Observatory (Germany)

914705 An overview and the current status of instrumentation at the Large Binocular Telescope Observatory (Invited Paper) [9147-4]

R. M. Wagner, M. L. Edwards, O. Kuhn, D. Thompson, C. Veillet, Large Binocular Telescope Observatory (United States)

914706 SOFIA science instruments: commissioning, upgrades and future opportunities [9147-5] E. C. Smith, NASA Ames Research Ctr. (United States); J. W. Miles, L. Helton, R. Sankrit, B. G. Andersson, E. E. Becklin, J. M. De Buizer, Universities Space Research Association (United States); C. D. Dowell, Jet Propulsion Lab. (United States); E. W. Dunham, Lowell Observatory (United States); R. Güsten, Max-Planck-Institut für Radioastronomie (Germany); D. A. Harper, The Univ. of Chicago (United States); T. L. Herter, Cornell Univ. (United States); L. D. Keller, Ithaca College (United States); R. Klein, Universities Space Research Association (United States); A. Krabbe, Univ. Stuttgart (Germany); S. Logsdon, Univ. of California, Los Angeles (United States); P. M. Marcum, NASA Ames Research Ctr. (United States);

I. S. McLean, Univ. of California, Los Angeles (United States); W. T. Reach, Universities Space Research Association (United States); M. J. Richter, Univ. of California, Davis (United States); T. L. Roellig, NASA Ames Research Ctr. (United States); G. Sandell, M. L. Savage, Universities Space Research Association (United States); P. Temi, NASA Ames Research Ctr. (United States); W. D. Vacca, J. E. Vaillancourt, J. E. Van Cleve, E. T. Young, Universities Space Research Association (United States) 
914707 The Daniel K. Inouye Solar Telescope first light instruments and critical science plan [9147-6] D. F. Elmore, T. Rimmele, National Solar Observatory (United States); R. Casini, National Ctr. for Atmospheric Research (United States); S. Hegwer, National Solar Observatory (United States); J. Kuhn, H. Lin, Institute for Astronomy, Univ. of Hawai'i (United States); J. P. McMullin, K. Reardon, National Solar Observatory (United States); W. Schmidt, Kiepenheuer-Institut für Sonnenphysik (Germany); A. Tritschler, F. Wöger, National Solar Observatory (United States)

\section{SESSION 2 NEW INSTRUMENTS AND UPGRADES TO EXISTING INSTRUMENTS}

914709 Introducing CUBES: the Cassegrain U-band Brazil-ESO spectrograph [9147-8] P. Bristow, European Southern Observatory (Germany); B. Barbuy, Univ. de São Paulo (Brazil); V. B. Macanhan, B. Castilho, Lab. Nacional de Astrofísica (Brazil); H. Dekker, B. Delabre, European Southern Observatory (Germany); M. Diaz, Univ. de São Paulo (Brazil); C. Gneiding, Lab. Nacional de Astrofísica (Brazil); F. Kerber, H. Kuntschner, European Southern Observatory (Germany); G. La Mura, Univ. degli Studi di Padova (Italy); R. Reiss, J. Vernet, European Southern Observatory (Germany)

9147 OA LRS2: the new facility low resolution integral field spectrograph for the Hobby-Eberly telescope [9147-9]

T. S. Chonis, G. J. Hill, H. Lee, S. E. Tuttle, B. L. Vattiat, The Univ. of Texas at Austin (United States)

9147 OB Project status of the Robert Stobie spectrograph near infrared instrument (RSS-NIR) for SALT [9147-10]

M. J. Wolf, M. P. Mulligan, M. P. Smith, D. P. Adler, C. M. Bartosz, M. A. Bershady, Univ. of Wisconsin-Madison (United States); D. A. H. Buckley, South African Astronomical Observatory (South Africa); M. P. Burse, P. A. Chordia, Inter-Univ. Ctr. for Astronomy and Astrophysics (India); J. C. Clemens, The Univ. of North Carolina at Chapel Hill (United States); H. W. Epps, Lick Observatory, Univ. of California, Santa Cruz (United States); K. Garot, B. L. Indahl, K. P. Jaehnig, Univ. of Wisconsin-Madison (United States); R. J. Koch, Diron Technologies, Inc. (United States); W. P. Mason, G. Mosby Jr., K. H. Nordsieck, J. W. Percival, Univ. of Wisconsin-Madison (United States); S. Punnadi, A. N. Ramaprakash, Inter-Univ. Ctr. for Astronomy and Astrophysics (India); J. A. Schier, The Pilot Group (United States); A. I. Sheinis, Australian Astronomical Observatory (Australia); S. A. Smee, Johns Hopkins Univ. (United States); D. J. Thielman, M. W. Werner, Univ. of Wisconsin-Madison (United States); T. B. Williams, South African Astronomical Observatory (South Africa); J. P. Wong, Paradigm Design Inc. (United States)

9147 OC VISIR upgrade overview and status [9147-11]

F. Kerber, H. Käufl, European Southern Observatory (Germany); P. Baksai, European Southern Observatory (Chile); N. Di Lieto, D. Dobrzycka, P. Duhoux, G. Finger, European Southern Observatory (Germany); S. Heikamp, Leiden Observatory (Netherlands); D. Ives, G. Jakob, L. Lundin, European Southern Observatory (Germany); D. Mawet, European Southern Observatory (Chile); L. Mehrgan, European Southern Observatory (Germany); Y. Momany, European Southern Observatory (Chile); V. Moreau, E. Pantin, CEA-Ctr. de SACLAY (France); M. Riquelme, European Southern Observatory (Chile); S. Sandrock, R. Siebenmorgen, A. Smette, J. Taylor, M. van den Ancker, European Southern Observatory (Germany); G. Valdes, European Southern Observatory (Chile); L. Venema, ASTRON (Netherlands); U. Weilenmann, European Southern Observatory (Chile) 
9147 OD The camera of the ASTRI SST-2M prototype for the Cherenkov Telescope Array [9147-12] O. Catalano, M. C. Maccarone, C. Gargano, G. La Rosa, A. Segreto, G. Sottile, INAF - Istituto di Astrofisica Spaziale e Fisica Cosmica di Palermo (Italy); V. De Caprio, INAF - Osservatorio Astronomico di Capodimonte (Italy); F. Russo, M. Capalbi, P. Sangiorgi, INAF - Istituto di Astrofisica Spaziale e Fisica Cosmica di Palermo (Italy); G. Bonanno, A. Grillo, S. Garozzo, D. Marano, S. Billotta, G. Romeo, INAF - Osservatorio Astrofisico di Catania (Italy); L. Stringhetti, M. Fiorini, N. La Palombara, S. Incorvaia, G. Toso, INAF - IASF Milano (Italy); D. Impiombato, S. Giarrusso, INAF - Istituto di Astrofisica Spaziale e Fisica Cosmica di Palermo (Italy)

9147 OE A two-dimensional spectropolarimeter as a first-light instrument for the Daniel K. Inouye Solar Telescope [9147-13]

W. Schmidt, A. Bell, C. Halbgewachs, F. Heidecke, T. J. Kentischer, O. von der Lühe, T. Scheiffelen, M. Sigwarth, Kiepenhever-Institut für Sonnenphysik (Germany)

9147 OF Performance of polarization modulation and calibration optics for the Daniel K. Inouye Solar Telescope [9147-14]

D. F. Elmore, National Solar Observatory (United States); S. R. Sueoka, College of Optical Sciences, The Univ. of Arizona (United States); R. Casini, National Ctr. for Atmospheric Research (United States)

9147 OG KIDSpec: an MKID based medium resolution integral field spectrograph [9147-332] K. O'Brien, N. Thatte, Univ. of Oxford (United Kingdom); B. Mazin, Univ. of California, Santa Barbara (United States)

$9147 \mathrm{OH} \quad$ HIPO in-flight performance improvements [9147-16] E. W. Dunham, T. A. Bida, P. L. Collins, G. Mandushev, S. Zoonematkermani, Lowell Observatory (United States); J. Van Cleve, Universities Space Research Association (United States); D. Angerhausen, Rensselaer Polytechnic Institute (United States); A. Mandell, NASA Goddard Space Flight Ctr. (United States)

9147 0J A near-infrared SETI experiment: instrument overview [9147-18]

S. A. Wright, Dunlap Institute for Astronomy and Astrophysics, Univ. of Toronto (Canada) and Univ. of Toronto (Canada); D. Werthimer, Univ. of California, Berkeley (United States); R. R. Treffers, Starman Systems, LLC (United States); J. Maire, Dunlap Institute for Astronomy and Astrophysics, Univ. of Toronto (Canada); G. W. Marcy, Univ. of California, Berkeley (United States); R. P. S. Stone, Lick Observatory, Univ. of California, Santa Cruz (United States); F. Drake, SETI Institute (United States); E. Meyer, P. Dorval, Univ. of Toronto (Canada); A. Siemion, Univ. of California, Berkeley (United States)

9147 OL Project overview and update on WEAVE: the next generation wide-field spectroscopy facility for the William Herschel Telescope [9147-20]

G. Dalton, STFC Rutherford Appleton Lab. (United Kingdom) and Univ. of Oxford (United Kingdom); S. Trager, Univ. of Groningen (Netherlands); D. C. Abrams, Isaac Newton Group of Telescopes (Spain); P. Bonifacio, GEPI, Observatoire de Paris à Meudon (France); J. A. L. Aguerri, Instituto de Astrofísica de Canarias (Spain); K. Middleton, STFC Rutherford Appleton Lab. (United Kingdom); C. Benn, K. Dee, Isaac Newton Group of Telescopes (Spain); F. Sayède, GEPI, Observatoire de Paris à Meudon (France); I. Lewis, Univ. of Oxford (United Kingdom); J. Pragt, NOVA ASTRON (Netherlands); S. Pico, Isaac Newton Group of 
Telescopes (Spain); N. Walton, Institute of Astronomy, Univ. of Cambridge (United Kingdom); J. Rey, Isaac Newton Group of Telescopes (Spain); C. Allende Prieto, J. Peñate, Instituto de Astrofísica de Canarias (Spain); E. Lhome, Isaac Newton Group of Telescopes (Spain); T. Agócs, NOVA ASTRON (Netherlands); J. Alonso, Instituto de Astrofisica de Canarias (Spain); D. Terrett, STFC Rutherford Appleton Lab. (United Kingdom); M. Brock, J. Gilbert, Univ. of Oxford (United Kingdom); A. Ridings, Isaac Newton Group of Telescopes (Spain); I. Guinouard, GEPI, Observatoire de Paris à Meudon (France); M. Verheijen, Univ. of Groningen (Netherlands); I. Tosh, K. Rogers, STFC Rutherford Appleton Lab. (United Kingdom); I. Steele, Liverpool John Moores Univ. (United Kingdom); R. Stuik, N. Tromp, NOVA ASTRON (Netherlands); A. Jasko, Konkoly Observatory (Hungary); J. Kragt, D. Lesman, NOVA ASTRON (Netherlands); C. Mottram, S. Bates, Liverpool John Moores Univ. (United Kingdom); F. Gribbin, Isaac Newton Group of Telescopes (Spain); L. F. Rodriguez, J. M. Delgado, Instituto de Astrofísica de Canarias (Spain); C. Martin, D. Cano, Isaac Newton Group of Telescopes (Spain); R. Navarro, NOVA ASTRON (Netherlands); M. Irwin, J. Lewis, E. Gonzalez Solares, Institute of Astronomy, Univ. of Cambridge (United Kingdom); N. O'Mahony, Isaac Newton Group of Telescopes (Spain); A. Bianco, INAF - Osservatorio Astronomico di Brera (Italy); C. Zurita, Instituto de Astrofisica de Canarias (Spain); R. ter Horst, NOVA ASTRON (Netherlands); E. Molinari, M. Lodi, J. Guerra, INAF - Fundación Galileo Galilei (Spain); A. Vallenari, A. Baruffolo, INAF - Osservatorio Astronomico di Padova (Italy)

9147 OM 4MOST: 4-metre Multi-Object Spectroscopic Telescope [9147-21]

R. S. de Jong, S. Barden, O. Bellido-Tirado, J. Brynnel, C. Chiappini, É. Depagne, R. Haynes, D. Johl, D. P. Phillips, O. Schnurr, A. D. Schwope, J. Walcher, S. M. Baver, G. Cescutti, M. R. Cioni, F. Dionies, H. Enke, Leibniz-Institut für Astrophysik Potsdam (Germany); D. Haynes, Leibniz-Institut für Astrophysik Potsdam (Germany) and InnoFSPEC (Germany); A. Kelz, F. S. Kitaura, G. Lamer, I. Minchev, V. Müller, S. E. Nuza, Leibniz-Institut für Astrophysik Potsdam (Germany); J.-C. Olaya, Leibniz-Institut für Astrophysik Potsdam (Germany) and InnoFSPEC (Germany); T. Piffl, E. Popow, A. Saviauk, M. Steinmetz, U. Ural, M. Valentini, R. Winkler, L. Wisotzki, Leibniz-Institut für Astrophysik Połsdam (Germany); W. R. Ansorge, RAMS-CON Management Consultants (Germany); M. Banerji, E. Gonzalez Solares, M. Irwin, R. C. Kennicutt Jr., D. P. King, R. McMahon, S. Koposov, I. R. Parry, X. Sun, N. A. Walton, Univ. of Cambridge (United Kingdom); G. Finger, O. Iwert, M. Krumpe, J.-L. Lizon, V. Mainieri, European Southern Observatory (Germany); J.-P. Amans, P. Bonifacio, M. Cohen, P. François, P. Jagourel, S. B. Mignot, F. Royer, P. Sartoretti, GEPI, Observatoire de Paris à Meudon, CNRS, Univ. Paris Diderot (France); R. Bender, H.-J. Hess, F. Lang-Bardl, B. Muschielok, J. Schlichter, Univ.-Sternwarte München (Germany); H. Böhringer, T. Boller, A. Bongiorno, M. Brusa, T. Dwelly, A. Merloni, K. Nandra, M. Salvato, Max-Planck-Institut für extraterrestrische Physik (Germany); J. H. Pragt, R. Navarro, G. Gerlofsma, R. Roelfsema, NOVA ASTRON (Netherlands); G. B. Dalton, Rutherford Appleton Lab. (United Kingdom) and Univ. of Oxford (United Kingdom); K. F. Middleton, I. A. Tosh, Rutherford Appleton Lab. (United Kingdom); C. Boeche, E. Caffau, N. Christlieb, E. K. Grebel, C. J. Hansen, A. Koch, H.-G. Ludwig, H. Mandel, A. Quirrenbach, L. Sbordone, W. Seifert, G. Thimm, Zentrum für Astronomie der Univ. Heidelberg (Germany); A. Helmi, S. C. trager, Kapteyn Astronomical Institute (Netherlands); T. Bensby, S. Feltzing, G. Ruchti, Lund Univ. (Sweden); B. Edvardsson, A. Korn, K. Lind, Uppsala Univ. (Sweden); W. Boland, NOVA (Netherlands); M. Colless, Australian Astronomical Observatory (Australia) and Australian National Univ. (Australia); G. Frost, J. Gilbert, P. Gillingham, J. Lawrence, N. Legg, W. Saunders, A. Sheinis, Australian Astronomical Observatory (Australia); S. Driver,

A. Robotham, Univ. of Western Australia (Australia); R. Bacon, P. Caillier, J. Kosmalski, F. Laurent, J. Richard, Ctr. de Recherche Astrophysique de Lyon (France) 
9147 ON MOONS: the Multi-Object Optical and Near-infrared Spectrograph for the VLT [9147-22]

M. Cirasuolo, UK Astronomy Technology Ctr. (United Kingdom) and Institute for Astronomy, Univ. of Edinburgh (United Kingdom); J. Afonso, Observatorio Astronomico de Lisboa (Portugal); M. Carollo, ETH Zürich (Switzerland); H. Flores, GEPI, Observatoire de Paris, CNRS, Univ. Paris Diderot (France); R. Maiolino, Univ. of Cambridge (United Kingdom); E. Oliva, INAF - Osservatorio Astrofisico di Arcetri (Italy); S. Paltani, Univ. of Genève (Switzerland); L. Vanzi, Pontificia Univ. Católica de Chile (Chile); C. Evans, UK Astronomy Technology Ctr. (United Kingdom); M. Abreu, Univ. de Lisboa (Portugal); D. Atkinson, UK Astronomy Technology Ctr. (United Kingdom); C. Babusiaux, GEPI, Observatoire de Paris, CNRS, Univ. Paris Diderot (France); S. Beard, UK Astronomy Technology Ctr. (United Kingdom); F. Baver, Pontificia Univ. Católica de Chile (Chile); M. Bellazzini, Univ. degli Studi di Bologna (Italy); R. Bender, Univ.-Sternwarte München (Germany) and Max-Planck-Institut für extraterrestrische Physik (Germany); P. Best, Institute for Astronomy, Univ. of Edinburgh (United Kingdom); N. Bezawada, UK Astronomy Technology Ctr. (United Kingdom); P. Bonifacio, GEPI, Observatoire de Paris, CNRS, Univ. Paris Diderot (France); A. Bragaglia, INAF - Osservatorio Astronomico di Bologna (Italy); I. Bryson, UK Astronomy Technology Ctr. (United Kingdom); D. Busher, Univ. of Cambridge (United Kingdom); A. Cabral, Univ. de Lisboa (Portugal); K. Caputi, Institute for Astronomy, Univ. of Edinburgh (United Kingdom); M. Centrone, INAF - Osservatorio Astronomico di Roma (Italy); F. Chemla, GEPI, Observatoire de Paris, CNRS, Univ. Paris Diderot (France); A. Cimatti, Univ. degli Studi di Bologna (Italy); M.-R. Cioni, Univ. of Hertfordshire (United Kingdom); G. Clementini, INAF Osservatorio Astronomico di Bologna (Italy); J. Coelho, Univ. de Lisboa (Portugal); D. Crnojevic, Institute for Astronomy, Univ. of Edinburgh (United Kingdom); E. Daddi, CEACtr. de SACLAY (France); J. Dunlop, Institute for Astronomy, Univ. of Edinburgh (United Kingdom); S. Eales, Cardiff Univ. (United Kingdom); S. Feltzing, Lund Observatory (Sweden); A. Ferguson, Institute for Astronomy, Univ. of Edinburgh (United Kingdom); M. Fisher, Univ. of Cambridge (United Kingdom); A. Fontana, INAF - Osservatorio Astronomico di Roma (Italy); J. Fynbo, Dark Cosmology Ctr. (Denmark); B. Garilli, INAF - IASF Milano (Italy); G. Gilmore, Univ. of Cambridge (United Kingdom); A. Glauser, ETH Zürich (Switzerland); I. Guinouard, F. Hammer, GEPI, Observatoire de Paris, CNRS, Univ. Paris Diderot (France); P. Hastings, UK Astronomy Technology Ctr. (United Kingdom); A. Hess, Univ.-Sternwarte München (Germany); R. Ivison, UK Astronomy Technology Ctr. (United Kingdom); P. Jagourel, GEPI, Observatoire de Paris, CNRS, Univ. Paris Diderot (France); M. Jarvis, Oxford Univ. (United Kingdom); L. Kaper, Astronomical Institute Anton Pannekoer (Netherlands); G. Kauffman, Max-Planck-Institut für Astrophysik (Germany); A. T. Kitching, Univ. College London (United Kingdom); A. Lawrence, Institute for Astronomy, Univ. of Edinburgh (United Kingdom); D. Lee, UK Astronomy Technology Ctr. (United Kingdom); B. Lemasle, Astronomical Institute Anton Pannekoer (Netherlands); G. Licausi, INAF - Osservatorio Astronomico di Roma (Italy); S. Lilly, ETH Zürich (Switzerland); D. Lorenzetti, INAF - Osservatorio Astronomico di Roma (Italy); D. Lunney, UK Astronomy Technology Ctr. (United Kingdom); R. Maiolino, Univ. of Cambridge (United Kingdom); F. Mannucci, INAF - Osservatorio Astrofisico di Arcetri (Italy); R. McLure, Institute for Astronomy, Univ. of Edinburgh (United Kingdom); D. Minniti, Pontificia Univ. Católica de Chile (Chile); D. Montgomery, UK Astronomy Technology Ctr. (United Kingdom); B. Muschielok, Univ.-Sternwarte München (Germany); K. Nandra, MaxPlanck-Institut für extraterrestrische Physik (Germany); R. Navarro, NOVA ASTRON (The Netherlands); P. Norberg, Durham Univ. (United Kingdom); S. Oliver, Univ. of Sussex (United Kingdom); L. Origlia, INAF - Osservatorio Astronomico di Bologna (Italy); N. Padilla, Pontificia Univ. Católica de Chile (Chile); J. Peacock, Institute for Astronomy, Univ. of Edinburgh (United Kingdom); F. Pedicini, INAF - Osservatorio Astronomico di Roma (Italy); J. Peng, Univ. of Cambridge (United Kingdom); L. Pentericci, INAF - Osservatorio Astronomico di Roma (Italy); J. Pragt, NOVA ASTRON (Netherlands); M. Puech, GEPI, Observatoire de Paris, CNRS, Univ. Paris Diderot (France); S. Randich, INAF - Osservatorio 
Astrofisico di Arcetri (Italy); P. Rees, UK Astronomy Technology Ctr. (United Kingdom); A. Renzini, INAF - Osservatorio Astronomico di Padova (Italy); N. Ryde, Lund Observatory (Sweden); M. Rodrigues, European Southern Observatory (Chile); I. Roseboom, Institute for Astronomy, Univ. of Edinburgh (United Kingdom); F. Royer, GEPI, Observatoire de Paris, CNRS, Univ. Paris Diderot (France); R. Saglia, Univ.-Sternwarte München (Germany) and Max-Planck-Institut für extraterrestrische Physik (Germany); A. Sanchez, Max-Planck-Institut für extraterrestrische Physik (Germany); R. Schiavon, Liverpool John Moores Univ. (United Kingdom); H. Schnetler, UK Astronomy Technology Ctr. (United Kingdom); D. Sobral, Institute for Astronomy, Univ. of Edinburgh (United Kingdom); R. Speziali, INAF - Osservatorio Astronomico di Roma (Italy); D. Sun, Univ. of Cambridge (United Kingdom); R. Stuik, NOVA ASTRON (Netherlands); A. Taylor, Institute for Astronomy, Univ. of Edinburgh (United Kingdom); W. Taylor, S. Todd, UK Astronomy Technology Ctr. (United Kingdom); E. Tolstoy, Kapteyn Astronomical Institute (Netherlands); M. Torres, Pontificia Univ. Católica de Chile (Chile); M. Tosi, E. Vanzella, INAF - Osservatorio Astronomico di Bologna (Italy); L. Venema, Kapteyn Astronomical Institute (Netherlands); F. Vitali, INAF - Osservatorio Astronomico di Roma (Italy); M. Wegner, Univ.-Sternwarte München (Germany); M. Wells, UK Astronomy Technology Ctr. (United Kingdom); V. Wild, St. Andrews Univ. (United Kingdom); G. Wright, UK Astronomy Technology Ctr. (United Kingdom); G. Zamorani, INAF - Osservatorio Astronomico di Bologna (Italy); M. Zoccali, Pontificia Univ. Católica de Chile (Chile)

914700 MEGARA: a new generation optical spectrograph for GTC [9147-23]

A. Gil de Paz, J. Gallego, Univ. Complutense de Madrid (Spain); E. Carrasco, Instituto Nacional de Astrofísica, Óptica y Electrónica (Mexico); J. Iglesias-Páramo, Instituto de Astrofísica de Andalucía (Spain); R. Cedazo, Univ. Politécnica de Madrid (Spain); J. M. Vílchez, Instituto de Astrofísica de Andalucía (Spain); M. L. García-Vargas, FRACTAL S.L.N.E (Spain); X. Arrillaga, M. A. Carrera, Added Value Solutions SL (Spain);

A. Castillo-Morales, Univ. Complutense de Madrid (Spain); E. Castillo-Domínguez, Instituto Nacional de Astrofísica, Óptica y Electrónica (Mexico); M. C. Eliche-Moral, Univ. Complutense de Madrid (Spain); D. Ferrusca, Instituto Nacional de Astrofísica, Óptica y Electrónica (Mexico); E. González-Guardia, Univ. Politécnica de Madrid (Spain); B. Lefort, M. Maldonado, FRACTAL S.L.N.E (Spain); R. A. Marino, Univ. Complutense de Madrid (Spain); I. Martínez-Delgado, FRACTAL S.L.N.E (Spain); I. Morales Durán, Instituto de Astrofísica de Andalucía (Spain); E. Mujica, FRACTAL S.L.N.E (Spain); G. Páez, Ctr. de Investigaciones en Óptica (Mexico); S. Pascual, Univ. Complutense de Madrid (Spain); A. Pérez-Calpena, FRACTAL S.L.N.E (Spain); A. Sánchez-Penim, Univ. Complutense de Madrid (Spain); E. Sánchez-Blanco, S. Tulloch, FRACTAL S.L.N.E (Spain); M. Velázquez, Instituto Nacional de Astrofísica, Óptica y Electrónica (Mexico); J. Zamorano, Univ. Complutense de Madrid (Spain); A. L. Aguerri, Instituto de Astrofísica de Canarias (Spain) and Univ. de La Laguna (Spain); D. Barrado y Naváscues, Ctr. de Astrobiología (Spain); E. Bertone, Instituto Nacional de Astrofísica, Óptica y Electrónica (Mexico); N. Cardiel, Univ. Complutense de Madrid (Spain); A. Cava, Univ. of Genève (Switzerland); J. Cenarro, Ctr. de Estudios de Física del Cosmos de Aragón (Spain); M. Chávez, Instituto Nacional de Astrofísica, Óptica y Electrónica (Mexico); M. García, Ctr. de Astrobiología (Spain); J. Guichard, Instituto Nacional de Astrofísica, Óptica y Electrónica (Mexico); R. Gúzman, Univ. of Florida (United States); A. Herrero, Instituto de Astrofísica de Canarias (Spain) and Univ. de La Laguna (Spain); N. Huélamo, Ctr. de Astrobiología (Spain); D. Hughes, Instituto Nacional de Astrofísica, Óptica y Electrónica (Mexico); J. Jiménez-Vicente, Univ. de Granada (Spain); C. Kehrig, I. Márquez, J. Masegosa, Instituto de Astrofísica de Andalucía (Spain); Y. D. Mayya, Instituto Nacional de Astrofísica, Óptica y Electrónica (Mexico); J. Méndez-Abreu, Univ. of St. Andrews (United Kingdom); M. Mollá, CIEMAT (Spain); C. Muñoz-Tuñón, Instituto de Astrofísica de Canarias (Spain) and Univ. de La Laguna (Spain); M. Peimbert, Univ. Autónoma de México (Mexico); P. G. Pérez-González, Univ. 
Complutense de Madrid (Spain); E. Pérez Montero, Instituto de Astrofísica de Andalucía (Spain); M. Rodríguez, Instituto Nacional de Astrofísica, Óptica y Electrónica (Mexico); J. M. Rodríguez-Espinosa, Instituto de Astrofísica de Canarias (Spain) and Univ. de La Laguna (Spain); L. Rodríguez-Merino, D. Rosa-González, Instituto Nacional de Astrofísica, Óptica y Electrónica (Mexico); J. Sánchez-Almeida, Instituto de Astrofísica de Canarias (Spain) and Univ. de La Laguna (Spain); C. Sánchez Contreras, Ctr. de Astrobiología (Spain); P. Sánchez-Blázquez, Univ. Autónoma de Madrid (Spain); F. M. Sánchez Moreno, Univ. Politécnica de Madrid (Spain); S. F. Sánchez, Univ. Autónoma de México (Mexico); A. Sarajedini, Univ. of Florida (United States); F. Serena, Univ. Politécnica de Madrid (Spain); S. Silich, Instituto Nacional de Astrofísica, Óptica y Electrónica (Mexico); S. Simón-Díaz, Instituto de Astrofísica de Canarias (Spain) and Univ. de La Laguna (Spain); G. Tenorio-Tagle, E. Terlevich, R. Terlevich, Instituto Nacional de Astrofísica, Óptica y Electrónica (Mexico); S. Torres-Peimbert, Univ. Autónoma de México (Mexico); I. Trujillo, Instituto de Astrofísica de Canarias (Spain) and Univ. de La Laguna (Spain); Y. Tsamis, Open Univ. (United Kingdom); O. Vega, Instituto Nacional de Astrofísica, Óptica y Electrónica (Mexico); V. Villar, Univ. Complutense de Madrid (Spain)

\section{SESSION 4 HIGH MULTIPLEX AND SURVEY INSTRUMENTS II}

9147 OP The WIYN one degree imager 2014: performance of the partially populated focal plane and instrument upgrade path [9147-24]

D. R. Harbeck, WIYN Observatory (United States); T. Boroson, National Optical Astronomy Observatory (United States) and Las Cumbres Observatory Global Telescope Network (United States); M. Lesser, The Univ. of Arizona (United States); J. Rajagopal, National Optical Astronomy Observatory (United States); A. Yeatts, WIYN Observatory (United States); C. Corson, National Optical Astronomy Observatory (United States); W. Liu, WIYN Observatory (United States); I. Dell'Antonio, Brown Univ. (United States); R. Kotulla, Univ. of Wisconsin-Milwaukee (United States) and Univ. of Wisconsin-Madison (United States); D. Ouellette, The Univ. of Arizona (United States); E. Hooper, WIYN Observatory (United States) and Univ. of Wisconsin-Madison (United States); M. Smith, Univ. of WisconsinMadison (United States); R. Bredthaver, Semiconductor Technology Associates, Inc. (United States); P. Martin, Univ. of Hawaili at Hilo (United States); G. Muller, GMTO Corp. (United States); P. Knezek, National Science Foundation (United States); M. Hunten, Lunar and Planetary Science Lab. (United States)

9147 OQ VIRUS: production and deployment of a massively replicated fiber integral field spectrograph for the upgraded Hobby-Eberly Telescope [9147-25]

G. J. Hill, McDonald Observatory, The Univ. of Texas at Austin (United States) and The Univ. of Texas at Austin (United States); S. E. Tuttle, N. Drory, H. Lee, B. L. Vattiat, McDonald Observatory, The Univ. of Texas at Austin (United States); D. L. DePoy, J. L. Marshall, Texas A\&M Univ. (United States); A. Kelz, D. Haynes, Leibniz-Institut für Astrophysik Potsdam (Germany) and innoFSPEC Potsdam (Germany); M. H. Fabricius, Max-Planck-Institut für extraterrestrische Physik (Germany); K. Gebhardt, The Univ. of Texas at Austin (United States); R. D. Allen, Texas A\&M Univ. (United States); H. Anwad, Institut für Astrophysik Göttingen (Germany); R. Bender, Max-Planck Institut für extraterrestrische Physik (Germany) and Univ.-Sternwarte München (Germany); G. Blanc, Carnegie Observatories (United States); T. Chonis, The Univ. of Texas at Austin (United States); M. E. Cornell, MIT Lincoln Lab. (United States); G. Dalton, Univ. of Oxford (United Kingdom); J. Good, McDonald Observatory, The Univ. of Texas at Austin (United States); T. Jahn, Leibniz-Institut für Astrophysik Potsdam (Germany) and innoFSPEC Potsdam (Germany); H. Kriel, M. Landriau, P. J. MacQueen, McDonald Observatory, The Univ. of Texas at Austin (United 
States); J. D. Murphy, Princeton Univ. (United States); T. W. Peterson, McDonald Observatory, The Univ. of Texas at Austin (United States); T. Prochaska, Texas A\&M Univ. (United States); H. Nicklas, Institut für Astrophysik Göttingen (Germany); J. Ramsey, McDonald Observatory, The Univ. of Texas at Austin (United States); M. M. Roth, LeibnizInstitut für Astrophysik Potsdam (Germany) and innoFSPEC Potsdam (Germany); R. D. Savage, McDonald Observatory, The Univ. of Texas at Austin (United States); J. Snigula, Max-Planck-Institut für extraterrestrische Physik (Germany)

9147 OR The construction, alignment, and installation of the VIRUS spectrograph [9147-26] S. E. Tuttle, G. J. Hill, H. Lee, B. Vattiat, E. Noyola, N. Drory, M. Cornell, T. Peterson, T. Chonis, McDonald Observatory, The Univ. of Texas at Austin (United States); R. Allen, Texas A\&M Univ. (United States); G. Dalton, Univ. of Oxford (United Kingdom); D. DePoy, Texas A\&M Univ. (United States); D. Edmonston, McDonald Observatory, The Univ. of Texas at Austin (United States); M. Fabricius, Max-Planck-Institut für extraterrestrische Physik (Germany); D. Haynes, A. Kelz, Leibniz-Institut für Astrophysik Potsdam (Germany); M. Landriau, McDonald Observatory, The Univ. of Texas at Austin (United States); M. Lesser, The Univ. of Arizona Imaging Technology Lab. (United States); B. Leach, Astronomical Research Cameras, Inc. (United States); J. Marshall, Texas A\&M Univ. (United States); J. Murphy, D. Perry, McDonald Observatory, The Univ. of Texas at Austin (United States); T. Prochaska, Texas A\&M Univ. (United States); J. Ramsey, R. Savage, McDonald Observatory, The Univ. of Texas at Austin

9147 OS The Dark Energy Spectroscopic Instrument (DESI) [9147-27]

B. Flaugher, Fermi National Accelerator Lab. (United States); C. Bebek, Lawrence Berkeley National Lab. (United States)

9147 OT Progress with the Prime Focus Spectrograph for the Subaru Telescope: a massively multiplexed optical and near-infrared fiber spectrograph [9147-28]

H. Sugai, N. Tamura, H. Karoji, A. Shimono, Kavli Institute for the Physics and Mathematics of the Universe, The Univ. of Tokyo (Japan); N. Takato, Subaru Telescope, National Astronomical Observatory of Japan (United States); M. Kimura, Y. Ohyama, Institute of Astronomy and Astrophysics, Academia Sinica (Taiwan); A. Ueda, National Astronomical Observatory of Japan (Japan); H. Aghazarian, Jet Propulsion Lab. (United States); M. V. de Arruda, Lab. Nacional de Astrofísica (Brazil); R. H. Barkhouser, C. L. Bennett, Johns Hopkins Univ. (United States); S. Bickerton, Kavli Institute for the Physics and Mathematics of the Universe, The Univ. of Tokyo (Japan); A. Bozier, Aix-Marseille Univ., CNRS, Lab. d'Astrophysique de Marseille (France); D. F. Braun, Jet Propulsion Lab. (United States); K. Bui, California Institute of Technology (United States); C. M. Capocasale, Jet Propulsion Lab. (United States); M. A. Carr, Princeton Univ. (United States); B. Castilho, Lab. Nacional de Astrofísica (Brazil); Y.-C. Chang, H.-Y. Chen, R. C. Y. Chou, Institute of Astronomy and Astrophysics, Academia Sinica (Taiwan); O. R. Dawson, Jet Propulsion Lab. (United States); R. G. Dekany, Caltech Optical Observatories (United States); E. M. Ek, Jet Propulsion Lab. (United States); R. S. Ellis, California Institute of Technology (United States); R. J. English, Jet Propulsion Lab. (United States); D. Ferrand, Aix-Marseille Univ., CNRS, Lab. d'Astrophysique de Marseille (France); D. Ferreira, Lab. Nacional de Astrofísica (Brazil); C. D. Fisher, Jet Propulsion Lab. (United States); M. Golebiowski, Aix-Marseille Univ., CNRS, Lab. d'Astrophysique de Marseille (France); J. E. Gunn, Princeton Univ. (United States); M. Hart, T. M. Heckman, Johns Hopkins Univ. (United States); P. T. P. Ho, Institute of Astronomy and Astrophysics, Academia Sinica (Taiwan); S. Hope, Johns Hopkins Univ. (United States); L. E. Hovland, Jet Propulsion Lab. (United States); S.-F. Hsu, Y.-S. Hu, P. J. Huang, Institute of Astronomy and Astrophysics, Academia Sinica (Taiwan); M. Jaquet, Aix-Marseille Univ., CNRS, Lab. d'Astrophysique de Marseille (France); J. E. Karr, Institute of Astronomy and 
Astrophysics, Academia Sinica (Taiwan); J. G. Kempenaar, M. E. King, Jet Propulsion Lab. (United States); O. Le Fèvre, D. Le Mignant, Aix-Marseille Univ., CNRS, Lab. d'Astrophysique de Marseille (France); H.-H. Ling, Institute of Astronomy and Astrophysics, Academia Sinica (Taiwan); C. Loomis, R. H. Lupton, Princeton Univ. (United States); F. Madec, Aix-Marseille Univ., CNRS, Lab. d'Astrophysique de Marseille (France); P. Mao, California Institute of Technology (United States); L. S. Marrara, Lab. Nacional de Astrofísica (Brazil); B. Ménard, Johns Hopkins Univ. (United States); C. Morantz, Jet Propulsion Lab. (United States); H. Murayama, Kavli Institute for the Physics and Mathematics of the Universe, The Univ. of Tokyo (Japan); G. J. Murray, Durham Univ. (United Kingdom); A. C. de Oliveira, Lab. Nacional de Astrofísica (Brazil); C. M. de Oliveira, Univ. de São Paulo (Brazil); L. S. de Oliveira, Lab. Nacional de Astrofísica (Brazil); J. D. Orndorff, Johns Hopkins Univ. (United States); R. de Paiva Vilaça, Lab. Nacional de Astrofísica (Brazil); E. J. Partos, Jet Propulsion Lab. (United States); S. Pascal, T. Pegot-Ogier, Aix-Marseille Univ., CNRS, Lab. d'Astrophysique de Marseille (France); D. J. Reiley, R. Riddle, California Institute of Technology (United States); L. Santos, J. B. dos Santos, Lab. Nacional de Astrofísica (Brazil); M. A. Schwochert, Jet Propulsion Lab. (United States); M. D. Seiffert, Jet Propulsion Lab. (United States) and California Institute of Technology (United States); S. A. Smee, Johns Hopkins Univ. (United States); R. M. Smith, Caltech Optical Observatories (United States); R. E. Steinkraus, Jet Propulsion Lab. (United States); L. Sodré Jr., Univ. de São Paulo (Brazil); D. N. Spergel, Princeton Univ. (United States); C. Surace, L. Tresse, C. Vidal, S. Vives, AixMarseille Univ., CNRS, Lab. d'Astrophysique de Marseille (France); S.-Y. Wang, C.-Y. Wen, Institute of Astronomy and Astrophysics, Academia Sinica (Taiwan); A. C. Wu, Jet Propulsion Lab. (United States); R. Wyse, Johns Hopkins Univ. (United States); C.-H. Yan, Institute of Astronomy and Astrophysics, Academia Sinica (Taiwan)

9147 OU Results of the verification of the NIR MOS EMIR [9147-29]

F. Garzón, Instituto de Astrofísica de Canarias (Spain) and Univ. de La Laguna (Spain); N. Castro-Rodríguez, M. Insausti, L. López-Martín, Instituto de Astrofísica de Canarias (Spain); P. Hammersley, Univ. de La Laguna (Spain); M. Barreto, P. Fernández, E. Joven, P. López, A. Mato, H. Moreno, M. Núñez, J. Patrón, J. L. Rasilla, P. Redondo, J. Rosich, Instituto de Astrofísica de Canarias (Spain); S. Pascual, European Southern Observatory (Germany); R. Grange, Lab. d'Astrophysique Marseille, CNRS (France)

9147 OV DOTIFS: a new multi-IFU optical spectrograph for the 3.6-m Devasthal optical telescope [9147-270]

H. Chung, Seoul National Univ. (Korea, Republic of) and Korea Institute for Advanced Study (Korea, Republic of); A. N. Ramaprakash, Inter-Univ. Ctr. for Astronomy and Astrophysics (India); A. Omar, Aryabhatta Research Institute of Observational Sciences (India); S. Ravindranath, S. Chattopadhyay, C. V. Rajarshi, P. Khodade, Inter-Univ. Ctr. for Astronomy and Astrophysics (India)

9147 OW Performance of the K-band multi-object spectrograph (KMOS) on the ESO VLT [9147-31] R. Sharples, Durham Univ. (United Kingdom); R. Bender, Univ.-Sternwarte München (Germany) and Max-Planck-Institut für extraterrestrische Physik (Germany);

A. Agudo Berbel, Max-Planck-Institut für extraterrestrische Physik (Germany); R. Bennett, N. Bezawada, UK Astronomy Technology Ctr. (United Kingdom); R. Castillo, European Southern Observatory (Germany); M. Cirasuolo, UK Astronomy Technology Ctr. (United Kingdom); P. Clark, Durham Univ. (United Kingdom); G. Davidson, UK Astronomy Technology Ctr. (United Kingdom); R. Davies, Max-Planck-Institut für extraterrestrische Physik (Germany); R. Davies, Univ. of Oxford (United Kingdom); M. Dubbeldam, Durham Univ. (United Kingdom); A. Fairley, UK Astronomy Technology Ctr. (United Kingdom); G. Finger, European Southern Observatory (Germany); N. F. Schreiber, R. Genzel, 
Max-Planck-Institut für extraterrestrische Physik (Germany); R. Haefner, A. Hess, Univ.-Sternwarte München (Germany); I. Jung, European Southern Observatory (Germany); I. Lewis, Univ. of Oxford (United Kingdom); D. Montgomery, J. Murray, UK Astronomy Technology Ctr. (United Kingdom); B. Muschielok, Univ.-Sternwarte München (Germany); J. Pirard, S. Ramsey, European Southern Observatory (Germany); P. Rees, UK Astronomy Technology Ctr. (United Kingdom); J. Richter, Univ.-Sternwarte München (Germany); D. Robertson, Durham Univ. (United Kingdom); I. Robson, UK Astronomy Technology Ctr. (United Kingdom); S. Rolt, Durham Univ. (United Kingdom); R. Saglia, Univ.-Sternwarte München (Germany); I. Saviane, European Southern Observatory (Germany); J. Schlichter, Univ.-Sternwarte München (Germany); L. Schmidtobreik, A. Segovia, A. Smette, European Southern Observatory (Germany); M. Tecza, Univ. of Oxford (United Kingdom); S. Todd, UK Astronomy Technology Ctr. (United Kingdom); M. Wegner, Univ.-Sternwarte München (Germany); E. Wiezorrek, Max-Planck-Institut für extraterrestrische Physik (Germany)

9147 OX Demonstration of high-performance cryogenic probe arms for deployable IFUs [9147-32]

S. S. Eikenberry, C. H. Murphey, S. A. Mullin, J. G. Bennett, S. N. Raines, K. Ackley, R. D. Stelter, A. Garner, Univ. of Florida (United States); J. Sabater, Univ. de Barcelona (Spain); S. A. Eikenberry, Eastside High School (United States); B. Chinn, H. V. Donoso, C. S. Vega, Univ. of Florida (United States); J. M. Gómez, J. Torra, Univ. de Barcelona (Spain); M. D. Herlevich, R. Frommeyer, P. Miller, Univ. of Florida (United States)

9147 OY First light results from the Hermes spectrograph at the AAT [9147-33]

A. Sheinis, Australian Astronomical Observatory (Australia) and The Univ. of Sydney (Australia); S. Barden, Leibniz-Institut für Astrophysik Potsdam (Germany); M. Birchall, Australian Astronomical Observatory (Australia); D. Carollo, Macquarie Univ. (Australia); J. Bland-Hawthorn, The Univ. of Sydney (Australia); J. Brzeski, S. Case, R. Cannon, V. Churilov, W. Couch, R. Dean, G. De Silva, Australian Astronomical Observatory (Australia); V. D'Orazi, Macquarie Univ. (Australia); T. Farrell, K. Fiegert, Australian Astronomical Observatory (Australia); K. Freeman, Australian National Univ. (Australia); G. Frost, L. Gers, M. Goodwin, D. Gray, R. Heald, Australian Astronomical Observatory (Australia); J. Heijmans, TNO (Netherlands); D. Jones, Prime Optics (Australia); S. Keller, The Australian National Univ. (Australia); U. Klauser, Y. Kondrat, Australian Astronomical Observatory (Australia); J. Lawrence, Australian Astronomical Observatory (Australia) and Macquarie Univ. (Australia); S. Lee, S. Mali, Australian Astronomical Observatory (Australia); S. Martell, Australian Astronomical Observatory (Australia) and The Univ. of New South Wales (Australia); D. Mathews, D. Mayfield, S. Miziarski, R. Muller, N. Pai, R. Patterson, E. Penny, D. Orr, K. Shortridge, Australian Astronomical Observatory (Australia); J. Simpson, Macquarie Univ. (Australia); S. Smedley, G. Smith, D. Stafford, N. Staszak, M. Vuong, L. Waller, Australian Astronomical Observatory (Australia); E. Wylie de Boer, Australian National Univ. (Australia); P. Xavier, J. Zheng, R. Zhelem, Australian Astronomical Observatory (Australia); D. Zucker, Macquarie Univ. (Australia)

\section{SESSION $5 \quad$ HIGH MULTIPLEX AND SURVEY INSTRUMENTS III}

$91470 Z$ KOSMOS and COSMOS: new facility instruments for the NOAO 4-meter telescopes [9147-34] P. Martini, The Ohio State Univ. (United States); J. Elias, National Optical Astronomy Observatory (United States); S. Points, Cerro Tololo Inter-American Observatory (Chile); D. Sprayberry, National Optical Astronomy Observatory (United States); M. A. Derwent, R. Gonzalez, J. A. Mason, T. P. O'Brien, D. P. Pappalardo, R. W. Pogge, The Ohio State Univ. (United States); R. Stoll, The Ohio State Univ. (United States) and Yale Univ. (United States); 
R. Zhelem, The Ohio State Univ (United States) and Australian Astronomical Observatory (Australia); P. Daly, M. Fitzpatrick, J. R. George, National Optical Astronomy Observatory (United States); M. Hunten, National Optical Astronomy Observatory (United States) and The Univ. of Arizona (United States); R. Marshall, G. Poczulp, S. Rath, R. Seaman, National Optical Astronomy Observatory (United States); M. Trueblood, National Optical Astronomy Observatory (United States) and Winer Observatory (United States); K. Zelaya, National Optical Astronomy Observatory (United States)

914710 TAIPAN: optical spectroscopy with StarBugs [9147-35]

K. Kuehn, J. Lawrence, D. M. Brown, S. Case, Australian Astronomical Observatory (Australia); M. Colless, The Australian National Univ. (Australia); R. Content, L. Gers, Australian Astronomical Observatory (Australia); J. Gilbert, Australian Astronomical Observatory (Australia) and Univ. of Oxford (United Kingdom); M. Goodwin, A. M. Hopkins, Australian Astronomical Observatory (Australia); M. Ireland, Australian National Univ. (Australia); N. P. F. Lorente, R. Muller, V. Nichani, A. Rakman, Australian Astronomical Observatory (Australia); S. N. Richards, The Univ. of Sydney (Australia); W. Saunders, N. F. Staszak, J. Tims, L. G. Waller, Australian Astronomical Observatory (Australia)

914712 mxSPEC: a massively multiplexed full-disk spectroheliograph for solar physics research [9147-37]

H. Lin, Institute for Astronomy, Univ. of Hawai'i (United States)

914713 BATMAN: a DMD-based multi-object spectrograph on Galileo telescope [9147-38] F. Zamkotsian, Lab. d'Astrophysique de Marseille, CNRS (France); P. Spano, INAF Osservatorio Astronomico di Brera (Italy); P. Lanzoni, H. Ramarijaona, Lab. d'Astrophysique de Marseille, CNRS (France); M. Moschetti, M. Riva, INAF - Osservatorio Astronomico di Brera (Italy); W. Bon, Lab. d'Astrophysique de Marseille, CNRS (France); L. Nicastro, INAF IASF Bologna (Italy); E. Molinari, R. Cosentino, A. Ghedina, M. Gonzalez, INAF - Telescopio Nazionale Galileo (Spain); P. Di Marcantonio, I. Coretti, R. Cirami, INAF - Osservatorio Astronomico di Trieste (Italy); F. Zerbi, INAF - Osservatorio Astronomico di Brera (Italy); L. Valenziano, INAF - IASF Bologna (Italy)

SESSION 6 HIGH SPECTRAL RESOLUTION INSTRUMENTS I

914714 Infrared Doppler instrument (IRD) for the Subaru telescope to search for Earth-like planets around nearby M-dwarfs [9147-39]

T. Kotani, National Astronomical Observatory of Japan (Japan); M. Tamura, National Astronomical Observatory of Japan (Japan) and The Univ. of Tokyo (Japan); H. Suto, National Astronomical Observatory of Japan (Japan); J. Nishikawa, National Astronomical Observatory of Japan (Japan) and The Graduate Univ. for Advanced Studies (Japan); B. Sato, Tokyo Institute of Technology (Japan); W. Aoki, T. Usuda, National Astronomical Observatory of Japan (Japan) and The Graduate Univ. for Advanced Studies (Japan); T. Kurokawa, National Astronomical Observatory of Japan (Japan) and Tokyo Univ. of Agriculture and Technology (Japan); K. Kashiwagi, Tokyo Univ. of Agriculture and Technology (Japan); S. Nishiyama, Miyagi Univ. of Education (Japan); Y. Ikeda, Photocoding (Japan); D. B. Hall, K. W. Hodapp, Institute for Astronomy, Univ. of Hawai'i (United States); J. Hashimoto, The Univ. of Oklahoma (United States); J. Morino, National Astronomical Observatory of Japan (Japan); Y. Okuyama, Y. Tanaka, S. Suzuki, S. Inove, Tokyo Univ. of Agriculture and Technology (Japan); J. Kwon, The Univ. of Tokyo (Japan); T. Suenaga, National Astronomical Observatory of Japan (Japan); D. Oh, The Graduate Univ. for Advanced Studies (Japan); H. Baba, National Astronomical Ovatory of Japan 
(Japan) and The Graduate Univ. for Advanced Studies (Japan); N. Narita, E. Kokubo, National Astronomical Observatory of Japan (Japan); Y. Hayano, Subaru Telescope, National Astronomical Observatory of Japan (United States); H. Izumiura, E. Kambe, National Astronomical Observatory of Japan (Japan); T. Kudo, Subaru Telescope, National Astronomical Observatory of Japan (United States); N. Kusakabe, National Astronomical Observatory of Japan (Japan); M. Ikoma, The Univ. of Tokyo (Japan); Y. Hori, Univ. of California Santa Cruz (United States); M. Omiya, National Astronomical Observatory of Japan (Japan); H. Genda, Tokyo Institute of Technology (Japan); A. Fukui, National Astronomical Observatory of Japan (Japan); Y. Fujii, Tokyo institute of Technology (Japan): O. Guyon, Subaru Telescope, National Astronomical Observatory of Japan (United States); H. Harakawa, M. Hayashi, National Astronomical Observatory of Japan (Japan); M. Hidai, Tokai Univ. (Japan); T. Hirano, M. Kuzuhara, Tokyo Institute of Technology (Japan); M. Machida, Kyusyu Univ. (Japan); T. Matsuo, T. Nagata, Kyoto Univ. (Japan); H. Onuki, National Astronomical Observatory of Japan (Japan); M. Ogihara, Nagoya Univ. (Japan); H. Takami, National Astronomical Observatory of Japan (Japan); N. Takato, Subaru Telescope, National Astronomical Observatory of Japan (United States); Y. H. Takahashi, National Astronomical Observatory of Japan (Japan); C. Tachinami, Tokyo Institute of Technology (Japan); H. Terada, National Astronomical Observatory of Japan (United States); H. Kawahara, The Univ. of Tokyo (Japan); T. Yamamuro, Optocraft (Japan)

914715 SPIRou: the near-infrared spectropolarimeter/high-precision velocimeter for the CanadaFrance-Hawaii telescope [9147-40]

É. Artigau, Univ. de Montréal (Canada); D. Kouach, J.-F. Donati, Institut de Recherche en Astrophysique et Planétologie, CNR, Univ. de Toulouse (France); R. Doyon, Univ. de Montréal (Canada); X. Delfosse, Institut de Planétologie et d'Astrophysique de Grenoble, CNRS, Univ. Joseph Fourier (France); S. Baratchart, M. Lacombe, Institut de Recherche en Astrophysique et Planétologie, CNR, Univ. de Toulouse (France); C. Moutou, Canada-France-Hawaii Telescope (United States); P. Rabou, Institut de Planétologie et d'Astrophysique de Grenoble, CNRS, Univ. Joseph Fourier (France); L. P. Parès, Y. Micheau, Institut de Recherche en Astrophysique et Planétologie, CNR, Univ. de Toulouse (France); S. Thibault, Univ. Laval (Canada); V. A. Reshetov, NRC - Herzburg Institute of Astrophysics (Canada); B. Dubois, Institut de Recherche en Astrophysique et Planétologie, CNR, Univ. de Toulouse (France); O. Hernandez, P. Vallée, Univ. de Montréal (Canada); S.-Y. Wang, Institute of Astronomy and Astrophysics, Academia Sinica (Taiwan); F. Dolon, Institut de Recherche en Astrophysique et Planétologie, CNR, Univ. de Toulouse (France); F. A. Pepe, Observatoire Astronomique de l'Univ. de Genève (Switzerland); F. Bouchy, Aix Marseille Univ., CNRS, Lab. d'Astrophysique de Marseille (Switzerland); N. Striebig, Institut de Recherche en Astrophysique et Planétologie, CNR, Univ. de Toulouse (France); F. Hénault, Institut de Planétologie et d'Astrophysique de Grenoble, CNRS, Univ. Joseph Fourier (France); D. Loop, L. Saddlemyer, NRC - Herzberg Institute of Astrophysics (Canada); G. Barrick, T. Vermeulen, Canada-France-Hawaii Telescope (United States); M. Dupieux, Institut de Recherche en Astrophysique et Planétologie, CNR, Univ. de Toulouse (France); G. Hébrard, Institut d'Astrophysique de Paris, CNRS, Univ. Pierre et Marie Curie (France); I. Boisse, Univ. do Porto (Portugal); E. Martioli, Lab. Nacional de Astrofísica (Brazil); S. H. P. Alencar, Univ. Federal de Minas Gerais (Brazil); J.-D. do Nascimento, Univ. Federal do Rio Grande do Norte (Brazil); P. Figueira, Univ. do Porto (Portugal)

914716 NRES: the network of robotic Echelle spectrographs [9147-41]

J. D. Eastman, Las Cumbres Observatory Global Telescope (United States) and Univ. of California, Santa Barbara (United States); T. M. Brown, Las Cumbres Observatory Global Telescope (United States) and Univ. of Colorado Boulder (United States); J. Hygelund, Las Cumbres Observatory Global Telescope (United States); J. van Eyken, Las Cumbres 
Observatory Global Telescope (United States) and Univ. of California, Santa Barbara (United States); J. R. Tufts, Las Cumbres Observatory Global Telescope (United States); S. Barnes, Stuart Barnes Optical Design (Netherlands)

914717 High resolution broad-band spectroscopy in the NIR using the Triplespec externally dispersed interferometer at the Hale telescope [9147-42]

D. J. Erskine, Lawrence Livermore National Lab. (United States); J. Edelstein, M. Sirk, E. Wishnow, Y. Ishikawa, E. McDonald, W. V. Shourt, Space Sciences Lab., Univ. of California, Berkeley (United States)

914718 A design for high-resolution spectroscopy with adaptive optics at the Large Binocular Telescope [9147-43]

R. O. Reynolds, L. Dettmann, J. C. Guerra, Large Binocular Telescope Observatory (United States)

914719 CRIRES+: a cross-dispersed high-resolution infrared spectrograph for the ESO VLT [9147-44] R. Follert, Thüringer Landessternwarte Tautenburg (Germany); R. J. Dorn, European Southern Observatory (Germany); E. Oliva, INAF - Osservatorio Astrofisico di Arcetri (Italy); J. L. Lizon, European Southern Observatory (Germany); A. Hatzes, Thüringer Landessternwarte Tautenburg (Germany); N. Piskunov, Uppsala Univ. (Sweden); A. Reiners, U. Seemann, Georg-August-Univ. Göttingen (Germany); E. Stempels, U. Heiter, T. Marquart, M. Lockhart, Uppsala Univ. (Sweden); G. Anglada-Escude, Georg-August-Univ. Göttingen (Germany); T. Löwinger, Thüringer Landessternwarte Tautenburg (Germany); D. Baade, J. Grunhut, P. Bristow, B. Klein, Y. Jung, D. J. Ives, F. Kerber, E. Pozna, J. Paufique, H. U. Kaeufl, European Southern Observatory (Germany); L. Origlia, INAF - Osservatorio Astronomico di Bologna (Italy); E. Valenti, D. Gojak, M. Hilker, L. Pasquini, A. Smette, J. Smoker, European Southern Observatory (Germany)

9147 1 A On-sky performance of a high resolution silicon immersion grating spectrometer [9147-45] J. Ge, S. Powell, B. Zhao, S. Schofield, F. Varosi, C. Warner, J. Liu, S. Sithajan, L. Avner, H. Jakeman, J. A. Gittelmacher, W. A. Yoder, Univ. of Florida (United States); M. Muterspaugh, M. Williamson, J. E. Maxwell, Tennessee State Univ. (United States)

9147 1C A laser frequency comb featuring sub- $\mathrm{cm} / \mathrm{s}$ precision for routine operation on HARPS [9147-47]

R. A. Probst, Max-Planck-Institut für Quantenoptik (Germany); G. Lo Curto, G. Avila, European Southern Observatory (Germany); B. L. Canto Martins, J. R. de Medeiros, Univ. Federal do Rio Grande do Norte (Brazil); M. Esposito, J. I. González Hernández, Instituto de Astrofísica de Canarias (Spain); T. W. Hänsch, Max-Planck-Institut für Quantenoptik (Germany); R. Holzwarth, Max-Planck-Institut für Quantenoptik (Germany) and Menlo Systems GmbH (Germany); F. Kerber, European Southern Observatory (Germany); I. C. Leão, Univ. Federal do Rio Grande do Norte (Brazil); A. Manescau, L. Pasquini, European Southern Observatory (Germany); R. Rebolo-López, Instituto de Astrofísica de Canarias (Spain); T. Steinmetz, Max-Planck-Institut für Quantenoptik (Germany) and Menlo Systems GmbH (Germany); T. Udem, Max-Planck-Institut für Quantenoptik (Germany); Y. Wu, Max-Planck-Institut für Quantenoptik (Germany) and Menlo Systems GmbH (Germany) 
9147 ID Design and early performance of IGRINS (Immersion Grating Infrared Spectrometer) [9147-48]

C. Park, Korea Astronomy and Space Science Institute (Korea, Republic of); D. T. Jaffe, The Univ. of Texas at Austin (United States); I.-S. Yuk, M.-Y. Chun, Korea Astronomy and Space Science Institute (Korea, Republic of); S. Pak, Kyung Hee Univ. (Korea, Republic of); K.-M. Kim, Korea Astronomy and Space Science Institute (Korea, Republic of); M. Pavel, H. Lee, The Univ. of Texas at Austin (United States); H. Oh, Korea Astronomy and Space Science Institute (Korea, Republic of) and Univ. of Science and Technology (Korea, Republic of); U. Jeong, Korea Astronomy and Space Science Institute (Korea, Republic of); C. K. Sim, H.-I. Lee, H. A. Nguyen Le, Kyung Hee Univ. (Korea, Republic of); J. Strubhar, M. Gully-Santiago, The Univ. of Texas at Austin (United States); J. S. Oh, S.-M. Cha, B. Moon, K. Park, Korea Astronomy and Space Science Institute (Korea, Republic of); C. Brooks, The Univ. of Texas at Austin (United States); K. Ko, J.-Y. Han, J. Nah, Korea Astronomy and Space Science Institute (Korea, Republic of); P. C. Hill, The Univ. of Texas at Austin (United States); S. Lee, Space Environment Lab. (Korea, Republic of); S. Barnes, Stuart Barnes Optical Design (Netherlands); Y. S. Yu, Korea Astronomy and Space Science Institute (Korea, Republic of); K. Kaplan, G. Mace, The Univ. of Texas at Austin (United States); H. Kim, J.-J. Lee, N. Hwang, Korea Astronomy and Space Science Institute (Korea, Republic of); B.-G. Park, Korea Astronomy and Space Science Institute (Korea, Republic of) and Univ. of Science and Technology (Korea, Republic of)

9147 IE High resolution near IR spectroscopy with GIANO-TNG [9147-49]

L. Origlia, INAF - Osservatorio Astronomico di Bologna (Italy); E. Oliva, C. Baffa, G. Falcini, E. Giani, F. Massi, INAF - Osservatorio Astrofisico di Arcetri (Italy); P. Montegriffo, INAF Osservatorio Astronomico di Bologna (Italy); N. Sanna, INAF - Osservatorio Astrofisico di Arcetri (Italy); S. Scuderi, INAF - Osservatorio Astrofisico di Catania (Italy); M. Sozzi, A. Tozzi, INAF - Osservatorio Astrofisico di Arcetri (Italy); I. Carleo, Bologna Univ. (Italy) and INAF Osservatorio Astrofisico di Padova (Italy); R. Gratton, INAF - Osservatorio Astrofisico di Padova (Italy); F. Ghinassi, M. Lodi, Telescopio Nazionale Galileo (Spain)

\section{SESSION 7 HIGH SPECTRAL RESOLUTION INSTRUMENTS II}

9147 IF CARMENES instrument overview [9147-50]

A. Quirrenbach, Landessternwarte Heidelberg (Germany); P. J. Amado, Instituto de Astrofísica de Andalucía (Spain); J. A. Caballero, Ctr. de Astrobiología (Spain); R. Mundt, Max-Planck-Institut für Astronomie (Germany); A. Reiners, Institut für Astrophysik Göttingen (Germany); I. Ribas, Institut de Ciències de l'Espai (Spain); W. Seifert, Landessternwarte Heidelberg (Germany); M. Abril, Instituto de Astrofísica de Andalucía (Spain); J. Aceituno, Calar Alto Observatory (Spain); F. J. Alonso-Floriano, Univ. Complutense de Madrid (Spain); M. Ammler-von Eiff, Thüringer Landessternwarte Tautenburg (Germany);

R. Antona Jiménez, Instituto de Astrofísica de Andalucía (Spain); H. Anwand-Heerwart, Institut für Astrophysik Göttingen (Germany); M. Azzaro, Calar Alto Observatory (Spain); F. Baver, Institut für Astrophysik Göttingen (Germany); D. Barrado, Calar Alto Observatory (Spain); S. Becerril, Instituto de Astrofísica de Andalucía (Spain); V. J. S. Béjar, Instituto de Astrofísica de Canarias (Spain); D. Benítez, Calar Alto Observatory (Spain); Z. M. Berdiñas, M. C. Cárdenas, E. Casal, A. Claret, Instituto de Astrofísica de Andalucía (Spain);

J. Colomé, Institut de Ciències de l'Espai (Spain); M. Cortés-Contreras, Univ. Complutense de Madrid (Spain); S. Czesla, Hamburger Sternwarte (Germany); M. Doellinger, Thüringer Landessternwarte Tautenburg (Germany); S. Dreizler, Institut für Astrophysik Göttingen (Germany); C. Feiz, Landessternwarte Heidelberg (Germany); M. Fernández, Instituto de Astrofísica de Andalucía (Spain); D. Galadí, Calar Alto Observatory (Spain); 
M. C. Gálvez-Ortiz, Ctr. de Astrobiología (Spain); A. García-Piquer, Institut de Ciéncies de I'Espai (Spain); M. L. García-Vargas, FRACTAL S.L.N.E (Spain); R. Garrido, Instituto de Astrofísica de Andalucía (Spain); L. Gesa, Institut de Ciències de l'Espai (Spain); V. Gómez Galera, Calar Alto Observatory (Spain); E. González Álvarez, Univ. Complutense de Madrid (Spain); J. I. González Hernández, Instituto de Astrofísica de Canarias (Spain); U. Grözinger, Max-Planck-Institut für Astronomie (Germany); J. Guàrdia, Institut de Ciències de l'Espai (Spain); E. W. Guenther, Thüringer Landessternwarte Tautenburg (Germany); E. de Guindos, Calar Alto Observatory (Spain); J. Gutiérrez-Soto, Instituto de Astrofísica de Andalucía (Spain); H.-J. Hagen, Hamburger Sternwarte (Germany); A. P. Hatzes, Thüringer Landessternwarte Tautenburg (Germany); P. H. Hauschildt, Hamburger Sternwarte (Germany); J. Helmling, Calar Alto Observatory (Spain); T. Henning, D. Hermann, MaxPlanck-Institut für Astronomie (Germany); L. Hernández Castaño, Calar Alto Observatory (Spain); E. Herrero, Institut de Ciències de I'Espai (Spain); D. Hidalgo, G. Holgado, Univ. Complutense de Madrid (Spain); A. Huber, Max-Planck-Institut für Astronomie (Germany); K. F. Huber, Hamburger Sternwarte (Germany); S. Jeffers, Institut für Astrophysik Göttingen (Germany); V. Joergens, Max-Planck-Institut für Astronomie (Germany); E. de Juan, Calar Alto Observatory (Spain); M. Kehr, Thüringer Landessternwarte Tautenburg (Germany); R. Klein, M. Kürster, Max-Planck-Institut für Astronomie (Germany); A. Lamert, Institut für Astrophysik Göttingen (Germany); S. Lalitha, Hamburger Sternwarte (Germany); W. Laun, Max-Planck-Institut für Astronomie (Germany); U. Lemke, Institut für Astrophysik Göttingen (Germany); R. Lenzen, Max-Planck-Institut für Astronomie (Germany); M. López del Fresno, B. López Martí, Ctr. de Astrobiología (Spain); J. López-Santiago, Univ. Complutense de Madrid (Spain); U. Mall, Max-Planck-Institut für Astronomie (Germany); H. Mandel, Landessternwarte Heidelberg (Germany); E. L. Martín, Ctr. de Astrobiología (Spain); S. Martín-Ruiz, Instituto de Astrofísica de Andalucía (Spain); H. Martínez-Rodríguez, Univ. Complutense de Madrid (Spain); C. J. Marvin, Institut für Astrophysik Göttingen (Germany); R. J. Mathar, Max-Planck-Institut für Astronomie (Germany); E. Mirabet, Instituto de Astrofísica de Andalucía (Spain); D. Montes, Univ. Complutense de Madrid (Spain); R. Morales Muñoz, Instituto de Astrofísica de Andalucía (Spain); A. Moya, Ctr. de Astrobiología (Spain); V. Naranjo, Max-Planck-Institut für Astronomie (Germany); A. Ofir, Institut für Astrophysik Göttingen (Germany); R. Oreiro, Instituto de Astrofísica de Andalucía (Spain); E. Pallé, Instituto de Astrofísica de Canarias (Spain); J. Panduro, Max-Planck-Institut für Astronomie (Germany); V.-M. Passegger, Institut für Astrophysik Göttingen (Germany); A. Pérez-Calpena, FRACTAL S.L.N.E (Spain); D. Pérez Medialdea, Instituto de Astrofísica de Andalucía (Spain); M. Perger, Institut de Ciències de l'Espai (Spain); M. Pluto, Thüringer Landessternwarte Tautenburg (Germany); A. Ramón, Instituto de Astrofísica de Andalucía (Spain); R. Rebolo, P. Redondo, Instituto de Astrofísica de Canarias (Spain); S. Reffert, Landessternwarte Heidelberg (Germany); S. Reinhardt, Calar Alto Observatory (Spain); P. Rhode, Institut für Astrophysik Göttingen (Germany); H.-W. Rix, F. Rodler, Max-PlanckInstitut für Astronomie (Germany); E. Rodríguez, C. Rodríguez-López, E. Rodríguez-Pérez, Instituto de Astrofísica de Andalucía (Spain); R.-R. Rohloff, Max-Planck-Institut für Astronomie (Germany); A. Rosich, Institut de Ciències de l'Espai (Spain); E. SánchezBlanco, M. A. Sánchez Carrasco, Instituto de Astrofísica de Andalucía (Spain); J. Sanz-Forcada, Ctr. de Astrobiología (Spain); L. F. Sarmiento, S. Schäfer, Institut für Astrophysik Göttingen (Germany); J. Schiller, Thüringer Landessternwarte Tautenburg (Germany); C. Schmidt, Institut für Astrophysik Göttingen (Germany); J. H. M. M. Schmitt, Hamburger Sternwarte (Germany); E. Solano, Ctr. de Astrobiología (Spain); O. Stahl, Landessternwarte Heidelberg (Germany); C. Storz, Max-Planck-Institut für Astronomie (Germany); J. Stürmer, Landessternwarte Heidelberg (Germany); J. C. Suárez, Instituto de Astrofísica de Andalucía (Spain); R.-G. Ulbrich, Institut für Astrophysik Göttingen (Germany); G. Veredas, K. Wagner, Landessternwarte Heidelberg (Germany); J. Winkler, Thüringer 
Landessternwarte Tautenburg (Germany); M. R. Zapatero Osorio, Ctr. de Astrobiología (Spain); M. Zechmeister, Institut für Astrophysik Göttingen (Germany);

F. J. Abellán de Paco, Univ. Complutense de Madrid (Spain); G. Anglada-Escudé, Univ. of London (United Kingdom); C. del Burgo, Instituto Nacional de Astrofísica, Óptica y Electrónica (Mexico); A. Klutsch, INAF - Osservatorio Astrofisico di Catania (Italy); J. L. Lizon, European Organisation for Astronomical Research in the Southern Hemisphere (Germany); M. López-Morales, Harvard-Smithsonian Ctr. for Astrophysics (United States); J. C. Morales, LESIA, Observatoire de Paris (France); M. A. C. Perryman, Univ. College Dublin (Ireland); S. M. Tulloch, FRACTAL S.L.N.E (Spain); W. XU, Wenli Xu Optical System Engineering (Germany)

9147 IG The Habitable-zone Planet Finder: A status update on the development of a stabilized fiberfed near-infrared spectrograph for the for the Hobby-Eberly telescope [9147-51] S. Mahadevan, L. W. Ramsey, R. Terrien, S. Halverson, A. Roy, F. Hearty, E. Levi, G. K. Stefansson, P. Robertson, C. Bender, C. Schwab, The Pennsylvania State Univ. (United States); M. Nelson, Univ. of Virginia (United States)

9147 1H ESPRESSO: the radial velocity machine for the VLT [9147-52]

D. Mégevand, Observatoire de l'Univ. de Genève (Switzerland); F. M. Zerbi, INAF Osservatorio Astronomico di Brera (Italy); P. Di Marcantonio, INAF - Osservatorio Astronomico di Trieste (Italy); A. Cabral, Univ. de Lisboa (Portugal); M. Riva, INAF Osservatorio Astronomico di Brera (Italy); M. Abreu, Univ. de Lisboa (Portugal); F. Pepe, Observatoire de l'Univ. de Genève (Switzerland); S. Cristiani, INAF - Osservatorio Astronomico di Trieste (Italy); R. Rebolo Lopez, Instituto de Astrofísica de Canarias (Spain); N. C. Santos, Univ. do Porto (Portugal); H. Dekker, European Southern Observatory (Germany); M. Aliverti, INAF - Osservatorio Astronomico di Brera (Italy); C. Allende, M. Amate, Instituto de Astrofísica de Canarias (Spain); G. Avila, European Southern Observatory (Germany); V. Baldini, INAF - Osservatorio Astronomico di Trieste (Italy); T. Bandy, Univ. Bern (Switzerland); P. Bristow, European Southern Observatory (Germany); C. Broeg, Univ. Bern (Switzerland); R. Cirami, INAF - Osservatorio Astronomico di Trieste (Italy); J. Coelho, Univ. de Lisboa (Portugal); P. Conconi, INAF - Osservatorio Astronomico di Brera (Italy); I. Coretti, G. Cupani, V. D'Odorico, INAF - Osservatorio Astronomico di Trieste (Italy); V. De Caprio, INAF - Osservatorio Astronomico di Brera (Italy); B. Delabre, R. Dorn, European Southern Observatory (Germany); P. Figueira, Univ. do Porto (Portugal); A. Fragoso, Instituto de Astrofísica de Canarias (Spain); S. Galeotta, INAF - Osservatorio Astronomico di Trieste (Italy); L. Genolet, Observatoire de l'Univ. de Genève (Switzerland); R. Gomes, Univ. de Lisboa (Portugal); J. González Hernández, Instituto de Astrofísica de Canarias (Spain); I. Hughes, Observatoire de I'Univ. de Genève (Switzerland); O. Iwert, F. Kerber, European Southern Observatory (Germany); M. Landoni, INAF - Osservatorio Astronomico di Brera (Italy); J.-L. Lizon, European Southern Observatory (Germany); C. Lovis, C. Maire, Observatoire de l'Univ. de Genève (Switzerland); M. Mannetta, INAF Osservatorio Astronomico di Trieste (Italy); C. C. J. A. P. Martins, Univ. do Porto (Portugal); P. Molaro, INAF - Osservatorio Astronomico di Trieste (Italy); M. A. S. Monteiro, Univ. do Porto (Portugal); M. Moschetti, INAF - Osservatorio Astronomico di Brera (Italy); A. Oliveira, Univ. de Lisboa (Portugal); M. R. Zapatero Osorio, Ctr. de Astrobiología (Spain); E. Poretti, INAF Osservatorio Astronomico di Brera (Italy); J. L. Rasilla, S. Santana Tschudi, Instituto de Astrofísica de Canarias (Spain); P. Santos, Univ. de Lisboa (Portugal); D. Sosnowska, Observatoire de l'Univ. de Genève (Switzerland); S. Sousa, Univ. do Porto (Portugal); F. Tenegi, Instituto de Astrofísica de Canarias (Spain); G. Toso, INAF - Osservatorio Astronomico di Brera (Italy); E. Vanzella, M. Viel, INAF - Osservatorio Astronomico di Trieste (Italy) 
$914711 \quad$ PIMMS échelle: the next generation of compact diffraction limited spectrographs for arbitrary input beams [9147-53]

C. H. Betters, S. G. Leon-Saval, J. Bland-Hawthorn, S. N. Richards, The Univ. of Sydney (Australia); T. A. Birks, I. Gris-Sánchez, Univ. of Bath (United Kingdom)

9147 iJ Progress on the Gemini High-Resolution Optical SpecTrograph (GHOST) design [9147-54] M. J. Ireland, Australian National Univ. (Australia); A. Anthony, G. Burley, E. Chisholm, NRC Herzberg Institute of Astrophysics (Canada); V. Churilov, Australian Astronomical Observatory (Australia); J. Dunn, NRC - Herzberg Institute of Astrophysics (Canada); G. Frost, J. Lawrence, Australian Astronomical Observatory (Australia); D. Loop, NRC Herzberg Institute of Astrophysics (Canada); P. McGregor, The Australian National Univ. (Australia); S. Martell, Univ. of New South Wales (Australia); A. McConnachie, NRC Herzberg Institute of Astrophysics (Canada); R. M. McDermid, Australian Astronomical Observatory (Australia) and Macquarie Univ. (Australia); J. Pazder, V. Reshetov, NRC Herzberg Institute of Astrophysics (Canada); J. G. Robertson, A. Sheinis, J. Tims, Australian Astronomical Observatory (Australia); P. Young, The Australian National Univ. (Australia); R. Zhelem, Australian Astronomical Observatory (Australia)

SESSION 8 HIGH SPATIAL RESOLUTION INSTRUMENTS I

$9147 \mathrm{KK}$ The integral field spectrograph for the Gemini planet imager [9147-55]

J. E. Larkin, J. K. Chilcote, T. Aliado, Univ. of California, Los Angeles (United States); B. J. Bauman, Lawrence Livermore National Lab. (United States); G. Brims, J. M. Canfield, Univ. of California, Los Angeles (United States); A. Cardwell, Gemini Observatory (Chile); D. Dillon, UARC, Univ. of California, Santa Cruz (United States); R. Doyon, Univ. de Montréal (Canada); J. Dunn, NRC - Herzberg Institute of Astrophysics (Canada); M. P. Fitzgerald, Univ. of California, Los Angeles (United States); J. R. Graham, Univ. of California, Berkeley (United States); S. Goodsell, M. Hartung, P. Hibon, Gemini Observatory (Chile); P. Ingraham, Kavli Institute for Particle Astrophysics and Cosmology, Stanford Univ. (United States) and Univ. de Montréal (Canada); C. A. Johnson, E. Kress, Univ. of California, Los Angeles (United States); Q. M. Konopacky, Dunlap Institute for Astronomy and Astrophysics, Univ. of Toronto (Canada); B. A. Macintosh, Lawrence Livermore National Lab. (United States) and Kavli Institute for Particle Astrophysics and Cosmology, Stanford Univ. (United States);

K. G. Magnone, Univ. of California, Los Angeles (United States); J. Maire, Dunlap Institute for Astronomy and Astrophysics, Univ. of Toronto (Canada); I. S. McLean, Univ. of California, Los Angeles (United States); D. Palmer, Lawrence Livermore National Lab. (United States); M. D. Perrin, Space Telescope Science Institute (United States); C. Quiroz, F. Rantakyrö, N. Sadakuni, Gemini Observatory (Chile); L. Saddlemyer, NRC - Herzberg Institute of Astrophysics (Canada); A. Serio, Gemini Observatory (Chile); S. Thibault, Univ. Laval (Canada); S. J. Thomas, NASA Ames Research Ctr. (United States) and UARC, Univ. of California, Santa Cruz (United States); P. Vallee, Univ. de Montréal (Canada); J. L. Weiss, Univ. of California, Los Angeles (United States)

9147 1L The SPHERE IFS at work [9147-56]

R. Claudi, E. Giro, M. Turatto, A. Baruffolo, INAF - Osservatorio Astronomico di Padova (Italy); P. Bruno, INAF - Osservatorio Astrofisico di Catania (Italy); E. Cascone, V. DeCaprio, INAF - Osservatorio Astronomico di Capodimonte (Italy); S. Desidera, INAF - Osservatorio Astronomico di Padova (Italy); R. Dorn, European Southern Observatory (Germany); D. Fantinel, INAF - Osservatorio Astronomico di Padova (Italy); G. Finger, European Southern Observatory (Germany); R. Gratton, L. Lessio, INAF - Osservatorio Astronomico di Padova (Italy); J. L. Lizon, European Southern Observatory (Germany); A. L. Maire, D. Mesa, 
B. Salasnich, INAF - Osservatorio Astronomico di Padova (Italy); S. Scuderi, INAF Osservatorio Astrofisico di Catania (Italy); A. Zurlo, INAF - Osservatorio Astronomico di Padova (Italy); K. Dohlen, Lab. d'Astrophysique de Marseille (France); J. L. Beuzit, D. Mouillet, P. Puget, Institut de Planétologie et d'Astrophysique de Grenoble (France); F. Wildi, Observatoire de Genève (Switzerland); N. Hubin, M. Kasper, European Southern Observatory (Germany)

$91471 \mathrm{M}$ The LINC-NIRVANA high resolution imager: challenges from the lab to first light [9147-57] T. M. Herbst, Max-Planck-Institut für Astronomie (Germany); R. Ragazzoni, INAF -

Osservatorio Astronomico di Padova (Italy); A. Eckart, Univ. zU Köln (Germany); G. Weigelt, Max-Planck-Institut für Radioastronomie (Germany)

914710 Operation and performance of the mid-infrared camera, NOMIC, on the Large Binocular Telescope [9147-59]

W. F. Hoffmann, P. M. Hinz, D. Defrère, J. M. Leisenring, A. J. Skemer, P. A. Arbo, M. Montoya, Steward Observatory, The Univ. of Arizona (United States); B. Mennesson, Jet Propulsion Lab. (United States)

9147 IP FRIDA, the diffraction limited NIR imager and IFS for the Gran Telescopio Canarias: status report [9147-60]

J. A. López, Univ. Nacional Autónoma de México (Mexico); J. Acosta, Instituto de Astrofísica de Canarias (Spain); L. C. Alvarez, Univ. Nacional Autónoma de México (Mexico); V. Bringas, Ctr. de Ingeniería y Desarrollo Industrial (Mexico); N. Cardiel, Univ. Complutense de Madrid (Spain); D. M. Clark, Univ. Nacional Autónoma de México (Mexico); A. Corrales, Ctr. de Ingeniería y Desarrollo Industrial (Mexico); S. Cuevas, O. Chapa, Univ. Nacional Autónoma de México (Mexico); J. J. Díaz, Instituto de Astrofísica de Canarias (Spain); S. S. Eikenberry, Univ. of Florida (United States); C. Eliche, Univ. Complutense de Madrid (Spain); C. Espejo, R. Flores, J. Fuentes, Univ. Nacional Autónoma de México (Mexico); J. Gallego, Univ. Complutense de Madrid (Spain); J. Garcés, Univ. Nacional Autónoma de México (Mexico); F. Garzón, Instituto de Astrofísica de Canarias (Spain); P. Hammersley, European Southern Observatory (Germany); C. Keiman, G. Lara, Univ. Nacional Autónoma de México (Mexico); P. López, Instituto de Astrofísica de Canarias (Spain); D. Lucero, Ctr. de Ingeniería y Desarrollo Industrial (Mexico); H. Moreno, Instituto de Astrofísica de Canarias (Spain); S. Pascual, Univ. Complutense de Madrid (Spain); J. Patrón, A. Prieto, Instituto de Astrofísica de Canarias (Spain); A. Rodríguez, B. Rodríguez, Ctr. de Ingeniería y Desarrollo Industrial (Mexico); B. Sánchez, Univ. Nacional Autónoma de México (Mexico); D. Torres, J. Uribe, Ctr. de Ingeniería y Desarrollo Industrial (Mexico); A. Watson, Univ. Nacional Autónoma de México (Mexico)

\section{Part Two}

$91471 Q$ Development and recent results from the Subaru coronagraphic extreme adaptive optics system [9147-61]

N. Jovanovic, Subaru Telescope, National Astronomical Observatory of Japan (United States); O. Guyon, Subaru Telescope, National Astronomical Observatory of Japan (United States) and Steward Observatory, The Univ. of Arizona (United States); F. Martinache, Observatoire de la Côte d'Azur (France); C. Clergeon, G. Singh, Subaru Telescope, National Astronomical Observatory of Japan (United States) and LESIA, Observatoire de Paris à Meudon (France); T. Kudo, Subaru Telescope, National Astronomical Observatory of Japan (United States); K. Newman, College of Optical Sciences, The Univ. of Arizona (United States) and NASA Ames Research Ctr. (United States); J. Kuhn, E. Serabyn, Jet 
Propulsion Lab. (United States); B. Norris, P. Tuthill, P. Stewart, The Univ. of Sydney (Australia); E. Huby, G. Perrin, S. Lacour, S. Vievard, LESIA, Observatoire de Paris à Meudon (France); N. Murakami, O. Fumika, Hokkaido Univ. (Japan); Y. Minowa, Y. Hayano, Subaru Telescope, National Astronomical Observatory of Japan (United States); J. White, Gemini Observatory (United States); O. Lai, Subaru Telescope, National Astronomical Observatory of Japan (United States); F. Marchis, SETI Institute (United States); G. Duchene, Univ. of California, Berkeley (United States) and Univ. Grenoble Alpes, CNRS, Institut de Planetologie et d'Astrophysique de Grenoble (France); T. Kotani, Subaru Telescope, National Astronomical Observatory of Japan (United States); J. Woillez, European Southern Observatory (Germany)

9147 IR High contrast polarimetry in the infrared with SPHERE on the VLT [9147-62] M. Langlois, Ctr. de Recherche Astronomique de Lyon, Observatoire de Lyon, CNRS, Univ. de Lyon 1 (France) and Ecole Normale Supérieure de Lyon (France); K. Dohlen, A. Vigan, A. Zurlo, C. Moutou, Aix-Marseille Univ., CNRS, Lab. d'Astrophysique de Marseille (France); H. M. Schmid, ETH Zürich (Switzerland); J. Mili, J.-L. Beuzit, Institut de Planétologie et d'Astrophysique de Grenoble (France); A. Boccaletti, LESIA, Observatoire de Paris (France); M. Carle, A. Costille, Aix-Marseille Univ., CNRS, Lab. d'Astrophysique de Marseille (France); R. Dorn, European Southern Observatory (Germany); L. Gluck, Institut de Planétologie et d'Astrophysique de Grenoble (France); N. Hubin, European Southern Observatory (Germany); M. Feldt, Max-Planck-Institut für Astronomie (Germany); M. Kasper, L. Lizon, European Southern Observatory (Germany); F. Madec, D. Le Mignant, Aix-Marseille Univ., CNRS, Lab. d'Astrophysique de Marseille (France); D. Mouillet, J.-P. Puget, Institut de Planétologie et d'Astrophysique de Grenoble (France); J.-F. Sauvage, ONERA (France); F. Wildi, Observatoire Astronomique de I'Univ. de Genève (Switzerland)

9147 is High-precision polarimetry at the Mont-Mégantic Observatory with the new polarimeter POMM [9147-63]

P. Bastien, O. Hernandez, L. Albert, É. Artigau, R. Doyon, Univ. de Montréal (Canada); L. Drissen, Univ. Laval (Canada); D. Lafrenière, A. F. J. Moffat, N. St-Louis, Univ. de Montréal (Canada)

9147 IT High-resolution imaging in the visible on large ground-based telescopes [9147-64] C. MacKay, Univ. of Cambridge (United Kingdom); R. Rebolo, Consejo Superior de Investigaciones Científicas (Spain); J. Crass, D. L. King, Univ. of Cambridge (United Kingdom); L. Labadie, Univ. zu Köln (Germany); V. González Escalera, M. Puga, Instituto de Astrofísica de Canarias (Spain); A. Pérez Garrido, Univ. Politécnica de Cartagena (Spain); R. López, A. Oscoz, J. A. Pérez-Prieto, L. F. Rodríguez-Ramos, S. Velasco, Instituto de Astrofísica de Canarias (Spain); I. Villó, Univ. Politécnica de Cartagena (Spain)

\section{SESSION 9 HIGH SPATIAL RESOLUTION INSTRUMENTS II}

9147 IU ERIS: preliminary design phase overview [9147-66]

H. Kuntschner, L. Jochum, P. Amico, J. K. Dekker, F. Kerber, E. Marchetti, M. Accardo, R. Brast, M. Brinkmann, R. D. Conzelmann, B.-A. Delabre, M. Duchateau, E. Fedrigo, G. Finger, C. Frank, F. G. Rodriguez, B. Klein, J. Knudstrup, M. Le Louarn, L. Lundin, A. Modigliani, M. Mueller, M. Neeser, S. Tordo, E. Valenti, European Southern Observatory (Germany); F. Eisenhaver, E. Sturm, H. Feuchtgruber, E. M. George, M. Hartl, R. Hofmann, H. Huber, M. P. Plattner, J. Schubert, K. Tarantik, E. Wiezorrek, Max-Planck-Institut für extraterrestrische Physik (Germany); M. R. Meyer, S. P. Quanz, A. M. Glauser, ETH Zürich (Switzerland); H. Weisz, Ingenieursbureau für den Maschinenbau (Germany); S. Esposito, 
9147 IV High-contrast planet imager for Kyoto 4m segmented telescope [9147-67]

T. Matsuo, Kyoto Univ. (Japan); N. Murakami, Hokkaido Univ. (Japan); T. Kotani, National Astronomical Observatory of Japan (Japan); H. Kawahara, The Univ. of Tokyo (Japan); N. Natsume, M. Kino, K. Yamamoto, Kyoto Univ. (Japan); H. Imada, Univ. of Tsukuba (Japan); M. Kurita, Kyoto Univ. (Japan); M. Iribe, H. Nishida, Osaka Electro-Communication Univ. (Japan); M. Kida, H. Kitou, Hokkaido Univ. (Japan); K. Ishikawa, RIKEN (Japan); Y. Uda, Osaka Electro-Communication Univ. (Japan); H. Tokoro, Nano-Optonics Research Institute (Japan); T. Nagata, F. Iwamuro, Kyoto Univ. (Japan); N. Miura, The Univ. of Tokyo (Japan); S. Oya, Subaru Telescope, National Astronomical Observatory of Japan (United States); Y. Itoh, Nishi-Harima Astronomical Observatory (Japan); H. Shibai, Osaka Univ. (Japan); M. Tamura, National Astronomical Observatory of Japan (Japan) and The Univ. of Tokyo (Japan)

9147 IW Construction and status of the CHARIS high contrast imaging spectrograph [9147-68] T. D. Groff, N. J. Kasdin, M. A. Limbach, M. Galvin, M. A. Carr, G. Knapp, T. Brandt, C. Loomis, N. Jarosik, Princeton Univ. (United States); K. Mede, National Astronomical Observatory of Japan (Japan); M. W. McElwain, NASA Goddard Space Flight Ctr. (United States); M. Janson, Princeton Univ. (United States); O. Guyon, N. Jovanovic, N. Takato, F. Martinache, Subaru Telescope, National Astronomical Observatory of Japan (United States); M. Hayashi, National Astronomical Observatory of Japan (Japan)

\section{SESSION 10 INSTRUMENTS FOR EXTREMELY LARGE TELESCOPES}

9147 IY Status of the instrumentation program for the Giant Magellan Telescope (Invited Paper) [9147-70]

G. H. Jacoby, A. Bouchez, Giant Magellan Telescope Organization (United States); M. Colless, The Australian National Univ. (Australia); D. DePoy, Texas A\&M Univ. (United States); D. Jaffe, The Univ. of Texas at Austin (United States); J. Lawrence, Australian Astronomical Observatory (Australia); P. McGregor, The Australian National Univ. (Australia); R. Bernstein, Giant Magellan Telescope Organization (United States) and Carnegie Observatories (United States); S. Shectman, Carnegie Observatories (United States); A. Szentgyorgyi, Harvard-Smithsonian Ctr. for Astrophysics (United States)

914712 The E-ELT instrument roadmap: a status report (Invited Paper) [9147-71] S. K. Ramsay, M. M. Casali, J. C. González, N. Hubin, European Southern Observatory (Germany)

914720 An update on the wide field, multi-object, moderate-resolution, spectrograph for the Giant Magellan Telescope [9147-72]

D. L. DePoy, R. Allen, T. Li, J. L. Marshall, C. Papovich, T. Prochaska, Texas A\&M Univ. (United States); S. Shectman, Carnegie Observatories (United States)

$914721 \quad$ METIS: the mid-infrared E-ELT imager and spectrograph [9147-73]

B. R. Brandl, Leiden Univ. (Netherlands); M. Feldt, Max-Planck-Institut für Astronomie (Germany); A. Glasse, UK Astronomy Technology Ctr. (United Kingdom); M. Guedel, Univ. Wien (Austria); S. Heikamp, M. Kenworthy, Leiden Univ. (Netherlands); R. Lenzen, 
Max-Planck-Institut für Astronomie (Germany); M. R. Meyer, ETH Zürich (Switzerland); F. Molster, NOVA (Netherlands) and Leiden Univ. (Netherlands); S. Paalvast, Janssen Precision Engineering BV (Netherlands); E. J. Pantin, Groupe LFEPS, Service d'Astrophysique (France); S. P. Quanz, ETH Zürich (Switzerland); E. Schmalzl, Leiden Univ. (Netherlands); R. Stuik, Leiden Univ. (Netherlands) and NOVA-ASTRON (Netherlands); L. Venema, Leiden Univ. (Netherlands) and ASTRON (Netherlands); C. Waelkens, Katholieke Univ. Leuven (Belgium)

914722 GMTNIRS (Giant Magellan Telescope Near-Infrared Spectrograph): optimizing the design for maximum science productivity and minimum risk [9147-74]

D. T. Jaffe, The Univ. of Texas at Austin (United States); S. Barnes, Stuart Barnes Optical Design (New Zealand); C. Brooks, M. Gully-Santiago, The Univ. of Texas at Austin (United States); S. Pak, Kyung Hee Univ. (Korea, Republic of); C. Park, I. Yuk, Korea Astronomy and Space Science Institute (Korea, Republic of)

914723 HIRES: the high resolution spectrograph for the E-ELT [9147-75] F. M. Zerbi, Scientific Directorate of INAF (Italy); F. Bouchy, Lab. d'Astrophysique de Marseille, CNRS (France); J. Fynbo, Dark Cosmology Ctr. (Denmark); R. Maiolino, Univ. of Cambridge (United Kingdom); N. Piskunov, Uppsala Univ. (Sweden); R. Rebolo Lopez, Instituto de Astrofísica de Canarias (Spain); N. Santos, Univ. do Porto (Portugal); K. Strassmeier, Leibniz-Institut für Astrophysik Potsdam (Germany); S. Udry, Observatoire Astronomique, Univ. de Genève (Switzerland); L. Vanzi, Pontificia Univ. Católica de Chile (Chile); M. Riva, INAF - Osservatorio Astronomico di Brera (Italy); A. Basden, Univ. of Durham (United Kingdom); I. Boisse, Lab. d'Astrophysique de Marseille, CNRS (France); X. Bonfils, Observatoire de Science de l'Univ. de Grenoble (France); D. Buscher, Univ. of Cambridge (United Kingdom); A. Cabral, Univ. de Lisboa (Portugal); P. Dimarcantonio, INAF -

Osservatorio Astronomico di Trieste (Italy); I. Di Varano, Observatoire Astronomique, Univ. de Genève (Switzerland); D. Henry, UK Astronomy Technology Ctr. (United Kingdom); M. Monteiro, Univ. do Porto (Portugal); T. Morris, G. Murray, Durham Univ. (United Kingdom): E. Oliva, INAF - Osservatorio Astrofisico di Arcetri (Italy); I. Parry, Univ. of Cambridge (United Kingdom); F. Pepe, Observatoire Astronomique, Univ. de Genève (Switzerland); A. Quirrenbach, Landessternwarte Heidelberg (Germany); J. L. Rasilla, Instituto de Astrofísica de Canarias (Spain); P. Rees, UK Astronomy Technology Ctr. (United Kingdom); E. Stempels, Uppsala Univ. (Sweden); L. Valenziano, INAF - IASF Bologna (Italy); M. Wells, UK Astronomy Technology Ctr. (United Kingdom); F. Wildi, Observatoire Astronomique, Univ. de Genève (Switzerland); L. Origlia, INAF - Osservatorio Astronomico di Bologna (Italy); C. Allende Prieto, Instituto de Astrofísica de Canarias (Spain); A. Chiavassa, Observatoire de la Côte d'Azur (France); S. Cristiani, INAF - Osservatorio Astronomico di Trieste (Italy); P. Figueira, Univ. do Porto (Portugal); B. Gustafsson, Uppsala Univ. (Sweden); A. Hatzes, Thüringer Landessternwarte Tautenburg (Germany); M. Haehnelt, Univ. of Cambridge (United Kingdom); K. Heng, Univ. Bern (Switzerland); G. Israelian, Instituto de Astrofísica de Canarias (Spain); O. Kochukhov, Uppsala Univ. (Sweden); C. Lovis, Observatoire Astronomique, Univ. de Genève (Switzerland); A. Marconi, Univ. degli Studi di Firenze (Italy); C. J. A. P. Martins, Univ. do Porto (Portugal); P. Noterdaeme, P. Petitjean, Institut d'Astrophysique de Paris (France); T. Puzia, Pontificia Univ. Católica de Chile (Chile); D. Queloz, Univ. of Cambridge (United Kingdom); A. Reiners, Georg-August-Univ. Göttingen (Germany); M. Zoccali, Pontificia Univ. Católica de Chile (Chile)

914724 The Infrared Imaging Spectrograph (IRIS) for TMT: instrument overview [9147-76] A. M. Moore, Caltech Optical Observatories (United States); J. E. Larkin, Univ. of California, Los Angeles (United States); S. A. Wright, Dunlap Institute for Astronomy and Astrophysics, Univ. of Toronto (Canada) and Univ. of Toronto (Canada); B. Bauman, Lawrence Livermore 
National Lab. (United States); J. Dunn, NRC - Herzberg Institute of Astrophysics (Canada); B. Ellerbroek, Thirty Meter Telescope Observatory Corp. (United States); A. C. Phillips, Univ. of California Observatories (United States); L. Simard, NRC - Dominion Astrophysical Observatory (Canada); R. Suzuki, National Astronomical Observatory of Japan (Japan); K. Zhang, Nanjing Institute of Astronomical Optics and Technology (China); T. Aliado, G. Brims, J. Canfield, Univ. of California, Los Angeles (United States); S. Chen, Dunlap Institute for Astronomy and Astrophysics, Univ. of Toronto (Canada); R. Dekany, A. Delacroix, Caltech Optical Observatories (United States); T. Do, Dunlap Institute for Astronomy and Astrophysics, Univ. of Toronto (Canada) and Univ. of Toronto (Canada); G. Herriot, NRC - Herzberg Institute of Astrophysics (Canada); B. Ikenoue, National Astronomical Observatory of Japan (Japan); C. Johnson, Univ. of California, Los Angeles (United States); E. Meyer, Dunlap Institute for Astronomy and Astrophysics, Univ. of Toronto (Canada) and Univ. of Toronto (Canada); Y. Obuchi, National Astronomical Observatory of Japan (Japan); J. Pazder, V. Reshetov, NRC - Herzberg Institute of Astrophysics (Canada); R. Riddle, Caltech Optical Observatories (United States); S. Saito, National Astronomical Observatory of Japan (Japan); R. Smith, Caltech Optical Observatories (United States); J. M. Sohn, Univ. of California, Los Angeles (United States); F. Uraguchi, T. Usuda, National Astronomical Observatory of Japan (Japan); E. Wang, Univ. of California, Los Angeles (United States); L. Wang, Thirty Meter Telescope Observatory Corp. (United States); J. Weiss, Univ. of California, Los Angeles (United States); R. Wooff, NRC Herzberg Institute of Astrophysics (Canada)

914725 HARMONI: the first light integral field spectrograph for the E-ELT [9147-77] N. A. Thatte, F. Clarke, Univ. of Oxford (United Kingdom); I. Bryson, H. Schnetler, UK Astronomy Technology Ctr. (United Kingdom); M. Tecza, Univ. of Oxford (United Kingdom); R. M. Bacon, A. Remillieux, CRAL, Observatoire de Lyon (France); E. Mediavilla, J. M. Herreros Linares, Instituto de Astrofísica de Canarias (Spain); S. Arribas, Ctr. de Astrobiologica (Spain); C. J. Evans, D. W. Lunney, UK Astronomy Technology Ctr. (United Kingdom); T. Fusco, ONERA (France) and Lab. d'Astrophysique de Marseille (France); K. O'Brien, Univ. of Oxford (United Kingdom); I. A. Tosh, STFC Rutherford Appleton Lab. (United Kingdom); D. J. Ives, G. Finger, European Southern Observatory (Germany); R. Houghton, R. L. Davies, J. D. Lynn, J. R. Allen, S. D. Zieleniewski, S. Kendrew, V. Ferraro-Wood, Univ. of Oxford (United Kingdom); A. Pécontal-Rousset, J. Kosmalski, J. Richard, A. Jarno, CRAL, Observatoire de Lyon (France); A. M. Gallie, D. M. Montgomery, D. Henry, UK Astronomy Technology Ctr. (United Kingdom); G. Zins, Institut de Planétologie et d'Astrophysique de Grenoble (France); D. Freeman, Kidger Optics Associates (United Kingdom); B. García-Lorenzo, L. F. Rodríguez-Ramos, J. S.-C. Revuelta, E. Hernández-Suárez, A. Bueno-Bueno, J. V. Gigante-Ripoll, Instituto de Astrofísica de Canarias (Spain); A. Garcia, SENER Ingeniería y Sistemas S.A. (Spain); K. Dohlen, B. Neichel, Lab. d'Astrophysique de Marseille (France)

914726 A preliminary design for the GMT-Consortium Large Earth Finder (G-CLEF) [9147-78] A. Szentgyorgyi, Harvard-Smithsonian Ctr. for Astrophysics (United States); S. Barnes, Stuart Barnes Optical Design (Netherlands); J. Bean, The Univ. of Chicago (United States); B. Bigelow, A. Bouchez, Giant Magellan Telescope Organization (United States); M.-Y. Chun, Korea Astronomy and Space Science Institute (Korea, Republic of); J. D. Crane, Carnegie Observatories (United States); H. Epps, Lick Observatory, Univ. of California, Santa Cruz (United States); I. Evans, J. Evans, Harvard-Smithsonian Ctr. for Astrophysics (United States); A. Frebel, MIT Kavli Institute for Astrophysics and Space Research (United States); G. Furesz, A. Glenday, Harvard-Smithsonian Ctr. for Astrophysics (United States); D. Guzman, Pontificia Univ. Católica de Chile (Chile); T. Hare, Carnegie Observatories (United States); B.-H. Jang, J.-G. Jang, U. Jeong, Korea Astronomy and 
Space Science Institute (Korea, Republic of); A. Jordan, MIT Kavli Institute for Astrophysics and Space Research (United States); K.-M. Kim, J. Kim, Korea Astronomy and Space Science Institute (Korea, Republic of); C.-H. Li, Stuart Barnes Optical Design (Netherlands); M. Lopez-Morales, K. McCracken, B. McLeod, M. Mueller, Harvard-Smithsonian Ctr. for Astrophysics (United States); J. Nah, Korea Astronomy and Space Science Institute (Korea, Republic of); T. Norton, Harvard-Smithsonian Ctr. for Astrophysics (United States); H. Oh, Korea Astronomy and Space Science Institute (Korea, Republic of) and Korea Univ. of Science and Technology (Korea, Republic of); J. S. Oh, Korea Astronomy and Space Science Institute (Korea, Republic of); M. Ordway, Harvard-Smithsonian Ctr. for Astrophysics (United States); B.-G. Park, Korea Astronomy and Space Science Institute (Korea, Republic of) and Korea Univ. of Science and Technology (Korea, Republic of); C. Park, S.-J. Park, Korea Astronomy and Space Science Institute (Korea, Republic of); D. Phillips, D. Plummer, W. Podgorski, F. Rodler, Harvard-Smithsonian Ctr. for Astrophysics (United States); A. Seifahrt, The Univ. of Chicago (United States); K.-M. Tak, Korea Astronomy and Space Science Institute (Korea, Republic of); A. Uomoto, Carnegie Observatories (United States); M. A. Van Dam, Flat Wavefronts (New Zealand); R. Walsworth, Harvard-Smithsonian Ctr. for Astrophysics (United States); Y. S. Yu, I.-S. Yuk, Korea Astronomy and Space Science Institute (Korea, Republic of)

914727 MOSAIC at the E-ELT: A multi-object spectrograph for astrophysics, IGM and cosmology [9147-79]

F. Hammer, GEPI, Observatoire de Paris, CNRS, Univ. Paris Diderot (France); B. Barbuy, Univ. de São Paulo (Brazil); J. G. Cuby, Aix Marseille Univ., CNRS, Lab. d'Astrophysique de Marseille (France); L. Kaper, Univ. van Amsterdam (Netherlands); S. Morris, Durham Univ. (United Kingdom); C. J. Evans, UK Astronomy Technology Ctr. (United Kingdom); P. Jagourel, GEPI, Observatoire de Paris, CNRS, Univ. Paris Diderot (France); G. Dalton, Univ. of Oxford (United Kingdom) and Rutherford Appleton Lab. (United Kingdom); P. Rees, UK Astronomy Technology Ctr. (United Kingdom); M. Puech, M. Rodrigues, GEPI, Observatoire de Paris, CNRS, Univ. Paris Diderot (France); D. Pearson, UK Astronomy Technology Ctr. (United Kingdom); K. Disseau, GEPI, Observatoire de Paris, CNRS, Univ. Paris Dideroł (France)

914728 Conceptual design of the MOBIE imaging spectrograph for TMT [9147-80]

B. C. Bigelow, Giant Magellan Telescope Organization (United States); M. V. Radovan, Univ. of California Observatories (United States); R. A. Bernstein, Giant Magellan Telescope Organization (United States); P. M. Onaka, H. Yamada, S. Isani, Institute for Astronomy, Univ. of Hawai'i (United States); S. Miyazaki, S. Ozaki, National Astronomical Observatory of Japan (Japan)

914729 Astrophotonic micro-spectrographs in the era of ELTs [9147-81]

N. Blind, Max-Planck-Institut für extraterrestrische Physik (Germany) and Institut de Planétologie et d'Astrophysique de Grenoble, Univ. J. Fourier, CNRS (France); E. Le Coarer, P. Kern, Institut de Planétologie et d'Astrophysique de Grenoble, Univ. J. Fourier, CNRS

(France); J. Bland-Hawthorn, The Univ. of Sydney (Australia)

POSTER SESSION: INSTRUMENT PROGRAMS AND NEW SCIENCE INSTRUMENTS AND UPGRADES

9147 2C Updated optical design and trade-off study for MOONS, the Multi-Object Optical and Near Infrared spectrometer for the VLT [9147-84]

E. Oliva, INAF - Osservatorio Astrofisico di Arcetri (Italy); S. Todd, M. Cirasuolo, H. Schnetler,

D. Lunney, P. Rees II, STFC UK Astronomy Technology Ctr. (United Kingdom); A. Bianco, 
INAF - Osservatorio Astronomico di Brera (Italy); E. Diolaiti, INAF - Osservatorio Astronomico di Bologna (Italy); D. Ferruzzi, INAF - Osservatorio Astrofisico di Arcetri (Italy); M. Fisher, Univ. of Cambridge (United Kingdom); I. Guinouard, GEPI, Observatoire de Paris, CNRS, Univ. Paris Diderot (France); M. IUzzolino, INAF - Osservatorio Astrofisico di Arcetri (Italy); I. Parry, $X$. Sun, Univ. of Cambridge (United Kingdom); A. Tozzi, INAF - Osservatorio Astrofisico di Arcetri (Italy); F. Vitali, INAF - Osservatorio Astronomico di Roma (Italy)

$91472 \mathrm{D}$ Optical, mechanical and electronic design and integration of POMM, a polarimeter for the Observatoire du mont Mégantic [9147-85]

M. R. Leclerc, P. Côté, F. Duchesne, INO (Canada); P. Bastien, Univ. de Montréal (Canada); O. Hernandez, Univ. de Montréal (Canada) and Observatoire du Mont Mégantic (Canada); P. Colonna d'Istria, Univ. de Montréal (Canada); M. Demers, M. Girard, M. Savard, D. Lemieux, INO (Canada); S. Thibault, D. Brousseau, Univ. Laval (Canada)

9147 2F A polarimetric unit for HARPS-North at the Telescopio Nazionale Galileo: HANPO [9147-87] F. Leone, Univ. degli Studi di Catania (Italy) and INAF - Osservatorio Astrofisico di Catania (Italy); M. Cecconi, R. Cosentino, A. Ghedina, INAF - Fundación Galileo Galilei (Spain); M. Giarrusso, Univ. degli Studi di Catania (Italy) and INAF - Osservatorio Astrofisico di Catania (Italy); M. Gonzalez, V. Lorenzi, INAF - Fundación Galileo Galilei (Spain); M. Munari, INAF - Osservatorio Astrofisico di Catania (Italy); H. Perez Ventura, L. Riverol, J. San Juan, INAF - Fundación Galileo Galilei (Spain); S. Scuderi, INAF - Osservatorio Astrofisico di Catania (Italy)

91472 A An off-the-shelf guider for the Palomar 200-inch telescope: interfacing amateur astronomy software with professional telescopes for an easy life [9147-88]

F. Clarke, J. Lynn, N. Thatte, M. Tecza, Univ. of Oxford (United Kingdom)

$91472 \mathrm{H} \quad$ Design updates and status of the fourth generation TripleSpec spectrograph [9147-89] E. Schlawin, T. L. Herter, C. Henderson, Cornell Univ. (United States); J. C. Wilson, Univ. of Virginia (United States); R. Probst, D. Sprayberry, National Optical Astronomy Observatory (United States); M. Bonati, P. Schurter, D. James, M. Warner, R. Tighe, Cerro Tololo InterAmerican Observatory (Chile); J. D. Adams, Univ. Research Space Association (United States); M. Martinez, Cerro Tololo Inter-American Observatory (Chile)

$91472 \mathrm{~J} \quad$ A Fabry-Perot and grism imaging spectrograph LISS (Line Imager and Slit Spectrograph) [9147-91]

Y. Hashiba, M. Doi, S. Sako, T. Morokuma, The Univ. of Tokyo (Japan); H. Kuncarayakti, Millennium Institute of Astrophysics (Chile) and Univ. de Chile (Chile); M. Kokubo, K. Mitsuda, H. Takahashi, K. Tateuchi, The Univ. of Tokyo (Japan); M. Watanabe, H. Nakao, Hokkaido Univ. (Japan)

$91472 \mathrm{~K}$ The mechanical design for the WEAVE prime focus corrector system [9147-93]

D. C. Abrams, K. Dee, Isaac Newton Group of Telescopes (Spain); T. Agócs, NOVA ASTRON (Netherlands); E. Lhome, Isaac Newton Group of Telescopes (Spain); J. Peñate, Instituto de Astrofísica de Canarias (Spain); A. Jaskó, E. Bányai, MTA Research Ctr. for Astronomy and Earth Sciences (Hungary); J. A. Burgal, Instituto de Astrofísica de Canarias (Spain); G. Dalton, STFC Rutherford Appleton Lab. (United Kingdom) and Univ. of Oxford (United Kingdom); K. Middleton, STFC Rutherford Appleton Lab. (United Kingdom); P. Bonifacio, GEPI, Observatoire de Paris à Meudon (France); J. A. L. Aguerri, Instituto de Astrofísica de Canarias (Spain); S. C. Trager, Univ. of Groningen (Netherlands); M. Balcells, Isaac Newton Group of Telescopes (Spain) 
$91472 \mathrm{~L}$ Conceptual design of a low resolution spectrograph for the Astronomical Observatory of Córdoba [9147-94]

D. Q. Nagasawa, J. L. Marshall, D. L. DePoy, N. Mondrik, Texas A\&M Univ. (United States)

9147 2M The GRAVITY spectrometers: design report of the optomechanics and active cryogenic mechanisms [9147-95]

M. Wiest, S. Yazici, Univ. zu Köln (Germany); S. Fischer, Deutsches Zentrum für Luft- und Raumfahrt e.V. (Germany); M. Thiel, Kayser-Threde GmbH (Germany); M. Haug,

Max-Planck-Institut für extraterrestrische Physik (Germany); C. Araujo-Hauck, Gemini Observatory (Chile); C. Straubmeier, I. Wank, Univ. zu Köln (Germany); F. Eisenhaver, MaxPlanck-Institut für extraterrestrische Physik (Germany); G. Perrin, Lab. d'Etudes Spatiales et d'Instrumentation en Astrophysique (France), LESIA, Observatoire de Paris, CNRS, UPMC, Univ. Paris Diderot (France), and Groupement d'Intérêt Scientifique PHASE (France); W. Brandner, Max-Planck-Institut für Astronomie (Germany); K. Perraut, Lab. d'Astrophysique Observatoire de Grenoble (France); A. Amorim, CENTRA-SIM (Portugal); M. Schöller, European Southern Observatory (Germany); A. Eckart, Univ. zu Köln (Germany) and MaxPlanck-Institut für Radioastronomie (Germany)

$91472 \mathrm{~N} \quad$ First-generation instrumentation for the Discovery Channel Telescope [9147-96] T. A. Bida, E. W. Dunham, P. Massey, H. G. Roe, Lowell Observatory (United States)

$91472 Q \quad P O M M:$ design of rotating mechanism and hexapod structure [9147-99]

P. Côté, M. Leclerc, M. Demers, INO (Canada); P. Bastien, O. Hernandez, Univ. de Montréal (Canada)

$91472 R \quad$ Testing fully depleted CCD [9147-100]

R. Casas, Institut de Ciències de l'Espai (Spain); L. Cardiel-Sas, Institut de Física d'Altes Energies (Spain); F. J. Castander, Institut de Ciències de l'Espai (Spain); J. Jiménez, Institut de Física d'Altes Energies (Spain); J. de Vicente, Ctr. de Investigaciones Energéticas, Medioambientales y Tecnológicas (Spain)

$91472 S \quad$ Readout electronics of physics of accelerating universe camera [9147-101] J. de Vicente, J. Castilla, Ctr. de Investigaciones Energéticas, Medioambientales y Tecnológicas (Spain); J. Jiménez, L. Cardiel-Sas, J. M. Illa, Institut de Física d'Altes Energies (Spain)

$91472 \mathrm{~T} \quad$ Acousto-optical imaging spectropolarimetric devices: new opportunities and developments [9147-102]

V. Y. Molchanov, S. P. Anikin, S. I. Chizhikov, K. B. Yushkov, O. Y. Makarov, National Univ. of Science and Technology "MISiS" (Russian Federation); A. M. Tatarnikov, S. A. Potanin, V. F. Esipov, Lomonosov Moscow State Univ. (Russian Federation)

$91472 \mathrm{~V}$ The near infrared camera for the Subaru Prime Focus Spectrograph [9147-104] S. A. Smee, Johns Hopkins Univ. (United States); J. E. Gunn, Princeton Univ. (United States); M. Golebiowski, R. Barkhouser, Johns Hopkins Univ. (United States); S. Vivès, S. Pascal, Aix Marseille Univ., CNRS, Lab. d'Astrophysique de Marseille (France); M. Carr, Princeton Univ. (United States); S. C. Hope, Johns Hopkins Univ. (United States); C. Loomis, Princeton Univ. (United States); M. Hart, Johns Hopkins Univ. (United States); H. Sugai, N. Tamura, A. Shimono, Kavli Institute for the Physics and Mathematics of the Universe, The Univ. of Tokyo (Japan) 
9147 2W Detector driver systems and photometric estimates for RIMAS [9147-105]

V. L. Toy, Univ. of Maryland, College Park (United States); A. S. Kutyrev, Univ. of Maryland, College Park (United States) and NASA Goddard Space Flight Ctr. (United States); E. I. Lyness, NASA Goddard Space Flight Ctr. (United States); M. Muench, Norwegian Univ. of Science and Technology (Norway); F. D. Robinson, G. N. Lotkin, NASA Goddard Space Flight Ctr. (United States); J. I. Capone, S. Veilleux, Univ. of Maryland, College Park (United States); S. H. Moseley, N. A. Gehrels, NASA Goddard Space Flight Ctr. (United States); S. N. Vogel, Univ. of Maryland, College Park (United States)

9147 2X FIFI-LS: the facility far-infrared spectrometer for SOFIA [9147-106] R. Klein, SOFIA-USRA, NASA Ames Research Ctr. (United States); S. Beckmann, A. Bryant, S. Colditz, C. Fischer, F. Fumi, Univ. Stuttgart (Germany); N. Geis, Max-Planck-Institut für extraterrestrische Physik (Germany); R. Hönle, A. Krabbe, Univ. Stuttgart (Germany); L. Looney, Univ. of Illinois at Urbana-Champaign (United States); A. Poglitsch, W. Raab, Max-Planck-Institut für extraterrestrische Physik (Germany); F. Rebell, Univ. Stuttgart (Germany); M. Savage, SOFIA-USRA, NASA Ames Research Ctr. (United States)

$91472 Y$ FLITECAM: early commissioning results [9147-108]

S. E. Logsdon, I. S. McLean, Univ. of California, Los Angeles (United States); E. E. Becklin, USRA, NASA Ames Research Ctr. (United States); E. W. Dunham, Lowell Observatory (United States); R. T. Hamilton, USRA, NASA Ames Research Ctr. (United States); C. A. Johnson, Univ. of California, Los Angeles (United States); J. W. Milburn, California Institute of Technology (United States); M. L. Savage, S. S. Shenoy, USRA, NASA Ames Research Ctr. (United States); E. C. Smith, NASA Ames Research Ctr. (United States); W. D. Vacca, USRA, NASA Ames Research Ctr. (United States)

$91472 Z$ One controlling and driving module based on FPGA in optical fiber positioning device [9147-111]

J. Guo, Y. Gu, Y. Jin, C. Zhai, Univ. of Science and Technology of China (China)

914730 Weather monitor station and $225 \mathrm{GHz}$ radiometer system installed at Sierra Negra: the Large Millimeter Telescope site [9147-112]

D. Ferrusca, J. Contreras R., Instituto Nacional de Astrofísica, Óptica y Electrónica (Mexico)

914732 Near-infrared wavelength calibration of astrophysical spectrographs with the emission spectrum of the $\mathrm{CN}$ molecule [9147-115]

A. Boesch, A. Reiners, Georg-August-Univ. Göttingen (Germany); P. F. Bernath, Old

Dominion Univ. (United States); A. Seifahrt, The Univ. of Chicago (United States)

914733 The characteristics and development status of the control and housekeeping electronics of FRIDA [9147-116]

J. J. Díaz, Instituto de Astrofísica de Canarias (Spain); R. Flores-Meza, B. Sánchez, Univ. Nacional Autónoma de México (Mexico); J. Patrón, Instituto de Astrofísica de Canarias (Spain)

914734 Fibre positioning concept for the WEAVE spectrograph at the WHT [9147-117]

I. J. Lewis, Univ. of Oxford (United Kingdom); G. B. Dalton, Univ. of Oxford (United Kingdom) and Rutherford Appleton Lab. (United Kingdom); M. Brock, J. Gilbert, Univ. of Oxford (United Kingdom); D. C. Abrams, Isaac Newton Group of Telescopes (Spain);

J. A. L. Aguerri, Instituto de Astrofísica de Canarias (Spain); P. Bonifacio, GEPI, Observatoire de Paris (France); K. Middleton, Rutherford Appleton Lab. (United Kingdom); S. C. Trager, Univ. of Groningen (Netherlands) 
914735 Precise angular positioning at 6K: the FIFI-LS grating assembly [9147-118]

F. Rebell, Univ. Stuttgart (Germany); W. Raab, Max-Planck-Institut für extraterrestrische Physik (Germany); S. Colditz, S. Beckmann, A. Bryant, C. Fischer, F. Fumi, Univ. Stuttgart (Germany); N. Geis, Max-Planck-Institut für extraterrestrische Physik (Germany); R. Hönle, Univ. Stuttgart (Germany); R. Klein, SOFIA-USRA (United States); A. Krabbe, Univ. Stuttgart (Germany) and Deutsches SOFIA Institut (Germany); L. Looney, Univ. of Illinois at UrbanaChampaign (United States); A. Poglitsch, Max-Planck-Institut für extraterrestrische Physik (Germany); S. Ragan, Max-Planck Institut fuer Astronomie (Germany); M. Savage, SOFIAUSRA, NASA Ames Research Ctr. (United States)

914736 Cryogenic optical systems for the rapid infrared imager/spectrometer (RIMAS) [9147-119] J. I. Capone, Univ. of Maryland, College Park (United States); D. A. Content, NASA Goddard Space Flight Ctr. (United States); A. S. Kutyrev, Univ. of Maryland, College Park (United States) and NASA Goddard Space Flight Ctr. (United States); F. D. Robinson, G. N. Lotkin, NASA Goddard Space Flight Ctr. (United States) and Global Science and Technology, Inc. (United States); V. L. Toy, S. Veilleux, Univ. of Maryland, College Park (United States); S. H. Moseley, N. A. Gehrels, NASA Goddard Space Flight Ctr. (United States); S. N. Vogel, Univ. of Maryland, College Park (United States)

914737 An update on the development of IO:I: a NIR imager for the Liverpool Telescope [9147-120] R. M. Barnsley, I. A. Steele, S. D. Bates, C. J. Mottram, Liverpool John Moores Univ. (United Kingdom)

914738 The precise measurement of the attenuation coefficients of various IR optical materials applicable to immersion grating [9147-121]

S. Kaji, Kyoto-Sangyo Univ. (Japan); Y. Sarugaku, Japan Aerospace Exploration Agency (Japan); Y. Ikeda, Photocoding (Japan) and Kyoto-Sangyo Univ. (Japan); N. Kobayashi, The Univ. of Tokyo (Japan); K. Nakanishi, S. Kondo, Kyoto-Sangyo Univ. (Japan); C. Yasui, The Univ. of Tokyo (Japan); H. Kawakita, Kyoto-Sangyo Univ. (Japan)

914739 Mechanical design of mounts for IGRINS focal plane arrays and field flattening lenses [9147-122]

J. S. Oh, C. Park, S.-M. Cha, I.-S. Yuk, K.-M. Kim, M.-Y. Chun, K. Ko, H. Oh, U. Jeong, J. Nah, Korea Astronomy and Space Science Institute (Korea, Republic of); H. Lee, McDonald Observatory, The Univ. of Texas at Austin (United States); M. Pavel, D. T. Jaffe, The Univ. of Texas at Austin (United States)

9147 3B ARNICA and LonGSp: the refurbishment of two near infrared instruments [9147-124] S. Koshida, L. Vanzi, D. Guzman, R. Leiva, Pontificia Univ. Católica de Chile (Chile); M. A. Bonati, Cerro Tololo Inter-American Observatory (Chile); R. L. Avilés, Pontificia Univ. Católica de Chile (Chile); C. Baffa, F. Palla, F. Mannucci, INAF - Osservatorio Astrofisico di Arcetri (Italy); T. C. Shen, Pontificia Univ. Católica de Chile (Chile) and Blue Shadows Astronomy and Engineering Ltd. (Chile); V. Suc, Pontificia Univ. Católica de Chile (Chile)

9147 3C Revised specifications and current development status of MIMIZUKU: the mid-infrared instrument for the TAO 6.5-m telescope [9147-125]

T. Kamizuka, T. Miyata, S. Sako, R. Ohsawa, K. Asano, M. Uchiyama, K. Okada, M. Uchiyama, T. Nakamura, I. Sakon, T. Onaka, The Univ. of Tokyo (Japan); H. Kataza, Japan Aerospace Exploration Agency (Japan); T. Aoki, The Univ. of Tokyo (Japan) and Kiso Observatory, The Univ. of Tokyo (Japan); M. Doi, N. M. Kato, K. Kawara, Y. Kitagawa, K. Kohno, M. Konishi, The Univ. of Tokyo (Japan); S. Koshida, Pontificia Univ. Católica de Chile (Chile); T. Minezaki, T. Morokuma, K. Motohara, The Univ. of Tokyo (Japan); T. Soyano, The Univ. of Tokyo (Japan) and Kiso Observatory, The Univ. of Tokyo (Japan); H. Takahashi, 
Y. Tamura, T. Tanabé, M. Tanaka, The Univ. of Tokyo (Japan); K. Tarusawa, The Univ. of Tokyo (Japan) and Kiso Observatory, The Univ. of Tokyo (Japan); K. Tateuchi, S. Todo, Y. Yoshii, The Univ. of Tokyo (Japan)

9147 3E Daytime site characterisation of La Palma, and its relation to night-time conditions [9147-128]

M. J. Townson, A. Kellerer, J. Osborn, T. Butterley, T. Morris, R. W. Wilson, Durham Univ. (United Kingdom)

$91473 G$ First successful deployment of the ZIMPOL-3 system at the GREGOR telescope [9147-130]

R. Ramelli, Istituto Ricerche Solari Locarno (Switzerland); D. Gisler, Istituto Ricerche Solari Locarno (Switzerland) and Kiepenhever-Institut für Sonnenphysik (Germany); M. Bianda, Istituto Ricerche Solari Locarno (Switzerland); N. Bello González, S. Berdyugina, D. Soltau, Kiepenhever-Institut für Sonnenphysik (Germany)

9147 31 MUSICa image slicer prototype at 1.5-m GREGOR solar telescope [9147-132]

A. Calcines, R. L. López, Instituto de Astrofísica de Canarias (Spain); M. Collados, Instituto de Astrofísica de Canarias (Spain) and Univ. de La Laguna (Spain); N. Vega Reyes, Instituto de Astrofísica de Canarias (Spain)

9147 3J Gemini Planet Imager observational calibrations I: overview of the GPI data reduction pipeline [9147-133]

M. D. Perrin, Space Telescope Science Institute (United States); J. Maire, Dunlap Institute for Astronomy and Astrophysics, Univ. of Toronto (Canada); P. Ingraham, Kavli Institute for Particle Astrophysics and Cosmology, Stanford Univ. (United States) and Univ. de Montréal (Canada); D. Savransky, Cornell Univ. (United States); M. Millar-Blanchaer, Univ. of Toronto (Canada); S. G. Wolff, Johns Hopkins Univ. (United States) and Space Telescope Science Institute (United States); J.-B. Ruffio, SETI Institute (United States); J. J. Wang, Univ. of California, Berkeley (United States); Z. H. Draper, Univ. of Victoria (Canada) and NRC Herzberg Institute of Astrophysics (Canada); N. Sadakuni, Gemini Observatory (Chile); C. Marois, NRC - Herzberg Institute of Astrophysics (Canada) and Univ. of Victoria (Canada); A. Rajan, Arizona State Univ. (United States); M. P. Fitzgerald, Univ. of California, Los Angeles (United States); B. Macintosh, Kavli Institute for Particle Astrophysics and Cosmology, Stanford Univ. (United States) and Lawrence Livermore National Lab. (United States); J. R. Graham, Univ. of California, Berkeley (United States); R. Doyon, Univ. de Montréal (Canada); J. E. Larkin, J. K. Chilcote, Univ. of California, Los Angeles (United States); S. J. Goodsell, Gemini Observatory (Chile); D. W. Palmer, Lawrence Livermore National Lab. (United States); K. Labrie, Gemini Observatory (United States); M. Beaulieu, Observatoire de la Côte d'Azur, Univ. de Nice Sophia-Antipolis (France); R. J. De Rosa, Arizona State Univ. (United States) and Univ. of Exeter (United Kingdom); A. Z. Greenbaum, Johns Hopkins Univ. (United States) and Space Telescope Science Institute (United States); M. Hartung, P. Hibon, Gemini Observatory (Chile); Q. Konopacky, Dunlap Institute for Astronomy and Astrophysics, Univ. of Toronto (Canada); D. Lafreniere, Univ. de Montréal (Canada); J.-F. Lavigne, ABB, Inc. (Canada); F. Marchis, SETI Institute (United States); J. Patience, Arizona State Univ. (United States); L. Pueyo, Space Telescope Science Institute (United States); F. T. Rantakyrö, Gemini Observatory (Chile); R. Soummer,

A. Sivaramakrishnan, Space Telescope Science Institute (United States); S. Thomas, NASA Ames Research Ctr. (United States) and Univ. of California, Santa Cruz (United States); K. Ward-Duong, Arizona State Univ. (United States); S. Wiktorowicz, Univ. of California, Santa Cruz (United States) 
9147 3K KOALA, a wide-field 1000 element integral-field unit for the Anglo-Australian Telescope: assembly and commissioning [9147-134]

R. Zhelem, J. Brzeski, S. Case, V. Churilov, S. Ellis, T. Farrell, A. Green, A. Heng, A. Horton, Australian Astronomical Observatory (Australia); M. Ireland, Mount Stromlo Observatory (Australia); D. Jones, Prime Optics (Australia); U. Klauser, J. Lawrence, S. Miziarski, D. Orr, N. Pai, N. Staszak, J. Tims, M. Vuong, L. Waller, P. Xavier, Australian Astronomical Observatory (Australia)

$91473 \mathrm{~L} \quad$ Single-lock: a stable Fabry-Perot based wavelength calibrator [9147-136] T. M. McCracken, C. A. Jurgenson, D. A. Fischer, R. A. Stoll, A. E. Szymkowiak, J. Bradford, Yale Univ. (United States); W. Rutter, Massachusetts Institute of Technology (United States)

$91473 \mathrm{~N} \quad 225 \mathrm{GHz}$ opacity measurements at Summit camp, Greenland, for the GreenLand Telescope (GLT) site testing [9147-138]

P. L. Martin-Cocher, K. Asada, S. Matsushita, M.-T. Chen, Institute of Astronomy and Astrophysics, Academia Sinica (Taiwan); P. T. P. Ho, Institute of Astronomy and Astrophysics, Academia Sinica (Taiwan) and Harvard-Smithsonian Ctr. for Astrophysics (United States);

C. Chen, Institute of Astronomy and Astrophysics, Academia Sinica (Taiwan)

914730 S4El (Spectral Sampling with Slicer for Stellar and Extragalactical Instrumentation): a newgeneration of 3D spectro-imager dedicated to night astronomy [9147-139] F. Sayède, M. Puech, GEPI, Observatoire de Paris à Meudon (France); P. Mein, LESIA, Observatoire de Paris (France); P. Bonifacio, GEPI, Observatoire de Paris à Meudon (France); J.-M. Malherbe, R. Galicher, LESIA, Observatoire de Paris (France); J.-P. Amans, G. Fasola, GEPI, Observatoire de Paris à Meudon (France)

9147 3P Development of Nayoro optical camera and spectrograph for 1.6-m Pirka telescope of Hokkaido University [9147-140]

H. Nakao, M. Watanabe, K. Sorai, Hokkaido Univ. (Japan); M. Yamada, Kobe Univ. (Japan); Y. Itoh, Univ. of Hyogo (Japan); S. Sako, T. Miyata, The Univ. of Tokyo (Japan)

9147 3Q Instrument control software based on LabVIEW for HARPS-N [9147-141]

X. Gao, A. Vick, UK Astronomy Technology Ctr. (United Kingdom); A. Glenday, HarvardSmithsonian Ctr. for Astrophysics (United States); M. Gonzalez, INAF - Fundación Galileo Galilei (Spain)

$91473 R \quad$ Optomecatronic design and integration of a high resolution equipment Berkut to the 1-meter class telescopes [9147-142]

R. Granados, R. López, A. Farah, Univ. Nacional Autónoma de México (Mexico)

914735 VIRUS instrument collimator assembly [9147-143]

J. L. Marshall, D. L. DePoy, T. Prochaska, R. D. Allen, P. Williams, J.-P. Rheault, T. Li,

D. Q. Nagasawa, C. Akers, D. Baker, E. Boster, C. Campbell, E. Cook, A. Elder, A. Gary, J. Glover, M. James, E. Martin, W. Meador, N. Mondrik, M. Rodriguez-Patino, S. Villanueva Jr., Texas A\&M Univ. (United States); G. J. Hill, S. Tuttle, B. Vattiat, H. Lee, McDonald Observatory, The Univ. of Texas at Austin (United States); T. S. Chonis, The Univ. of Texas at Austin (United States); G. B. Dalton, M. Tacon, Univ. of Oxford (United Kingdom)

9147 3T Reverse and concurrent engineering applied of a high resolution equipment Berkut for 1-meter class telescopes [9147-144]

R. López, R. Granados, A. Farah, Univ. Nacional Autónoma de México (Mexico) 
$91473 \mathrm{U}$ Remote and automatic small-scale observatories: experience with an all-sky fireball patrol camera [9147-145]

F. C. M. Bettonvil, NOVA ASTRON (Netherlands) and Univ. Leiden (Netherlands)

9147 3V Optical integration and verification of LINC-NIRVANA [9147-146]

J. Moreno-Ventas, H. Baumeister, T. Bertram, P. Bizenberger, F. Briegel, D. Greggio,

F. Kittmann, L. Marafatto, L. Mohr, K. Radhakrishnan, H. Schray, Max-Planck-Institut für

Astronomie (Germany)

$91473 \mathrm{~W}$ The ZIMPOL high contrast imaging polarimeter for SPHERE: system test results [9147-147]

R. Roelfsema, NOVA ASTRON (Netherlands); A. Bazzon, H. M. Schmid, ETH Zürich

(Switzerland); J. Pragt, NOVA ASTRON (Netherlands); D. Gisler, ETH Zürich (Switzerland);

C. Dominik, Astronomical Institute Anton Pannekoek (Netherlands); A. Baruffolo, INAF -

Osservatorio Astronomico di Padova (Italy); J.-L. Beuzit, IPAG, Univ. Joseph Fourier (France);

A. Costille, K. Dohlen, Lab. d'Astrophysique de Marseille, CNRS, Univ. de Provence (France);

M. Downing, European Southern Observatory (Germany); E. Elswijk, M. de Haan, NOVA

ASTRON (Netherlands); N. Hubin, M. Kasper, European Southern Observatory (Germany);

C. Keller, Leiden Observatory (Netherlands); J.-L. Lizon, European Southern Observatory

(Germany); D. Mouillet, IPAG, Univ. Joseph Fourier (France); A. Pavlov, Max-Planck-Institut

für Astronomie (Germany); P. Puget, IPAG, Univ. Joseph Fourier (France); B. Salasnich, INAF

- Osservatorio Astronomico di Padova (Italy); J.-F. Sauvage, ONERA (France); F. Wildi,

Observatoire Astronomique de l'Univ. de Genève (Switzerland)

$91473 X \quad$ PANIC in the lab: status before commissioning [9147-148]

B. Dorner, A. Huber, Max-Planck-Institut für Astronomie (Germany);

M. C. Cárdenas Vázquez, I. M. Ferro Rodriguez, Instituto de Astrofísica de Andalucía (Spain); P. Bizenberger, V. Naranjo, J. Panduro, U. Mall, M. Alter, R. Mathar, C. Storz, R.-R. Rohloff, P. Fopp, W. Laun, Max-Planck-Institut für Astronomie (Germany); J. M. Ibáñez,

A. J. García Segura, V. Terrón, Instituto de Astrofísica de Andalucía (Spain); J. W. Fried, Max-Planck-Institut für Astronomie (Germany); M. Fernández, J. F. Rodríguez Gómez, Instituto de Astrofísica de Andalucía (Spain); K. Meisenheimer, Max-Planck-Institut für Astronomie (Germany)

\section{Part Three}

9147 3Y PISCO: the Parallel Imager for Southern Cosmology Observations [9147-149]

B. Stalder, A. A. Stark, S. M. Amato, J. Geary, Harvard-Smithsonian Ctr. for Astrophysics (United States); S. A. Shectman, Carnegie Observatories (United States); C. W. Stubbs, Harvard Univ. (United States) and Harvard-Smithsonian Ctr. for Astrophysics (United States); A. Szentgyorgyi, Harvard-Smithsonian Ctr. for Astrophysics (United States)

$91473 Z$ SITELLE optical design, assembly, and testing [9147-150]

D. Brousseau, S. Thibault, S. Fortin-Boivin, Univ. Laval (Canada); H. Zhang, ImmerVision (Canada); P. Vallée, Univ. de Montréal (Canada); H. Auger, L. Drissen, Univ. Laval (Canada)

914740 Automated alignment and on-sky performance of the Gemini planet imager coronagraph [9147-151]

D. Savransky, Cornell Univ. (United States); S. J. Thomas, NASA Ames Research Ctr. (United States); L. A. Poyneer, Lawrence Livermore National Lab. (United States); J. Dunn, NRC - Herzburg Institute of Astrophysics (Canada); B. A. Macintosh, Kavli Institute for 
Particle Astrophysics and Cosmology, Stanford Univ. (United States); N. Sadakuni, Gemini Observatory (Chile); D. Dillon, Univ. of California Observatories (United States) and Lick Observatory, Univ. of California, Santa Cruz (United States); S. J. Goodsell, M. Hartung, P. Hibon, F. Rantakyrö, A. Cardwell, A. Serio, Gemini Observatory (Chile)

914743 Better flat-fielding for ground-based UV spectrographs [9147-154]

F. Kerber, R. Hanuschik, S. Moehler, European Southern Observatory (Germany); A. Smette, J. Smoker, P. Bourget, European Southern Observatory (Chile); P. J. Dwyer, Energetiq Technology, Inc. (United States); M. Rotschädl, Mountain Photonics GmbH (Germany)

914744 Developing micro DC-brushless motor driver and position control for fiber positioners [9147-155]

L. Jenni, P. Hörler, L. Makarem, J.-P. Kneib, D. Gillet, H. Bleuler, M. Bouri, Ecole Polytechnique Fédérale de Lausanne (Switzerland); F. Prada, G. De Rivera, Univ. Autónoma de Madrid (Spain); J. Sanchez, Instituto de Astrofísica de Andalucía (Spain)

914745 A miniature cryogenic scanning Fabry-Perot interferometer for mid-IR to submm astronomical observations [9147-156]

S. C. Parshley, E. M. Vavagiakis, T. Nikola, G. J. Stacey, Cornell Univ. (United States)

914746 The development of ground-based infrared multi-object spectrograph based on the microshutter array [9147-157]

D.-S. Moon, Univ. of Toronto (Canada); S. Sivanandam, Dunlap Institute of Astronomy and Astrophysics, Univ. of Toronto (Canada); A. S. Kutyrev, S. H. Moseley Jr., NASA Goddard Space Flight Ctr. (United States); J. R. Graham, Univ. of California, Berkeley (United States); A. Roy, Indian Institute of Technology, Kharagpur (India)

914747 Optical design of the SUMIRe/PFS spectrograph [9147-158]

S. Pascal, S. Vives, Aix Marseille Univ., CNRS, Lab. d'Astrophysique de Marseille (France);

R. Barkhouser, Johns Hopkins Univ. (United States); J. E. Gunn, Princeton Univ. (United States)

914748 Spherical grating spectrometers [9147-159]

D. O'Donoghue, South African Astronomical Observatory (South Africa); J. C. Clemens, The Univ. of North Carolina at Chapel Hill (United States)

914749 Inverse analysis method to optimize the optic tolerances of MEGARA: the future IFU and multi-object spectrograph for GTC [9147-161]

R. Ortiz, E. Carrasco, Instituto Nacional de Astrofísica, Óptica y Electrónica (Mexico); G. Páez, Ctr. de Investigaciones en Óptica, A.C. (Mexico); E. Sánchez-Blanco, FRACTAL S.L.N.E (Spain); A. Gil de Paz, J. Gallego, Univ. Complutense de Madrid (Spain); R. Cedazo, Univ. Politécnica de Madrid (Spain); J. Iglesias-Páramo, Instituto de Astrofísica de Andalucía (Spain)

$91474 \mathrm{~A}$ Status and first results of the Canarias infrared camera experiment (CIRCE) for the Gran Telescopio Canarias [9147-162]

A. Garner, R. D. Stelter, S. S. Eikenberry, Univ. of Florida (United States); N. Lasso-Cabrera, Ctr. de Estudios de Física del Cosmos de Aragón (Spain); S. N. Raines, Univ. of Florida (United States); M. Charcos, NASA, SOFIA (United States); M. Edwards, Large Binocular Telescope Observatory (United States); A. Marín-Franch, Ctr. de Estudios de Física del Cosmos de Aragón (Spain); K. Ackley, Univ. of Florida (United States); A. J. Cenarro, Ctr. de Estudios de Física del Cosmos de Aragón (Spain); J. G. Bennett, B. Chinn, R. Frommeyer, 
M. D. Herlevich, P. Miller, C. H. Murphey, Univ. of Florida (United States); C. Packham, The Univ. of Texas at San Antonio (United States)

9147 4B LINC-NIRVANA: Diffraction limited optics in cryogenic environment [9147-163]

P. Bizenberger, H. Baumeister, P. Fopp, T. Herbst, W. Laun, L. Mohr, J. Moreno-Ventas, Max-Planck-Institut für Astronomie (Germany)

9147 4C A comparison of concepts for a photonic spectrograph [9147-164]

R. J. Harris, J. R. Allington-Smith, Durham Univ. (United Kingdom); D. MacLachlan,

R. R. Thomson, Scottish Universities Physics Alliance (United Kingdom)

9147 4D MSE spectrograph optical design: a novel pupil slicing technique [9147-165]

P. Spanò, NRC - Herzberg Institute of Astrophysics (Canada)

9147 4E New GRISMs for AFOSC based on volume phase holographic gratings in photopolymers [9147-166]

A. Zanutta, Politecnico di Milano (Italy) and INAF - Osservatorio Astronomico di Brera (Italy);

A. Bianco, M. Landoni, INAF - Osservatorio Astronomico di Brera (Italy); L. Tomasella,

S. Benetti, E. Giro, INAF - Osservatorio Astronomico di Padova (Italy)

$91474 \mathrm{~F}$ Characterization of the reflectivity of various black materials [9147-167]

J. L. Marshall, P. Williams, J.-P. Rheault, T. Prochaska, R. D. Allen, D. L. DePoy,

Texas A\&M Univ. (United States)

$91474 \mathrm{G}$ FIFI-LS observation planning and data reduction [9147-168]

A. Bryant, C. Fischer, R. Hönle, S. Beckmann, S. Colditz, F. Fumi, Univ. Stuttgart (Germany);

N. Geis, Max-Planck-Institut für extraterrestrische Physik (Germany); C. Iserlohe, Univ. zu Köln (Germany); R. Klein, Universities Space Research Association (United States); A. Krabbe,

Univ. Stuttgart (Germany); L. Looney, Univ. of Illinois at Urbana-Champaign (United States); A. Poglitsch, W. Raab, Max-Planck-Institut für extraterrestrische Physik (Germany); S. Ragan, Max-Planck-Institut für Astronomie (Germany); F. Rebell, Univ. Stuttgart (Germany);

M. Savage, Universities Space Research Association (United States)

$91474 \mathrm{H} \quad$ Tests and procedures for optimizing EMIR cooling system [9147-169]

P. Fernández Izquierdo, Instituto de Astrofísica de Canarias (Spain); J. L. Lizon, European Southern Observatory (Germany); M. Á. Núñez Cagigal, J. Patrón Recio, M. Barreto Cabrera, F. Garzón López, Instituto de Astrofísica de Canarias (Spain)

$91474 \mathrm{~J}$ Design, alignment, and deployment of the Hobby Eberly Telescope prime focus instrument package [9147-172]

B. Vattiat, G. J. Hill, H. Lee, W. Moreira, N. Drory, J. Ramsey, L. Elliot, M. Landriau, D. M. Perry, R. Savage, H. Kriel, The Univ. of Texas at Austin (United States); M. Häuser, F. Mangold, Ludwig-Maximilians-Univ. München (Germany)

9147 4K A near-infrared SETI experiment: probability distribution of false coincidences [9147-173] J. Maire, Dunlap Institute for Astronomy and Astrophysics, Univ. of Toronto (Canada); S. A. Wright, Dunlap Institute for Astronomy and Astrophysics, Univ. of Toronto (Canada) and Univ. of Toronto (Canada); D. Werthimer, Univ. of California, Berkeley (United States); R. R. Treffers, Starman Systems, LLC (United States); G. W. Marcy, Univ. of California, Berkeley (United States); R. P. S. Stone, Lick Observatory, Univ. of California, Santa Cruz (United States); F. Drake, SETI Institute (United States); A. Siemion, Univ. of California, Berkeley (United States) 
$91474 \mathrm{M}$ Automatisms in EMIR instrument to improve operation, safety and maintenance [9147-175] P. Fernández Izquierdo, M. Núñez Cagigal, R. Barreto Rodríguez, N. Martínez Rey, S. Santana Tschudi, M. Barreto Cabrera, J. Patrón Recio, F. Garzón López, Instituto de Astrofísica de Canarias (Spain)

$914740 \quad$ HONIR: an optical and near-infrared simultaneous imager, spectrograph, and polarimeter for the 1.5-m Kanata telescope [9147-177]

H. Akitaya, Y. Moritani, T. Ui, T. Urano, Y. Ohashi, K. S. Kawabata, Hiroshima Univ. (Japan); A. Nakashima, Nagoya City Science Museum (Japan); M. Sasada, Kyoto Univ. (Japan); K. Sakimoto, T. Harao, Hiroshima Univ. (Japan); H. Miyamoto, Molex Japan Co., Ltd. (Japan); R. Matsui, R. Itoh, K. Takaki, I. Ueno, T. Ohsugi, Hiroshima Univ. (Japan); H. Nakaya, T. Yamashita, National Astronomical Observatory of Japan (Japan); M. Yoshida, Hiroshima Univ. (Japan)

9147 4Q PAUCam readout electronics assembly, integration and test (AIT) [9147-179]

J. Jiménez, J. M. Illa, L. Cardiel-Sas, Institut de Física d'Altes Energies (Spain); J. de Vicente,

J. Castilla, Ctr. de Investigaciones Energéticas, Medioambientales y Tecnológicas (Spain);

R. Casas, Institut de Ciències de l'Espai (Spain)

$91474 R \quad$ A flux calibration device for the SuperNova Integral Field Spectrograph (SNIFS) [9147-180] S. Lombardo, Univ. Bonn (Germany); G. Aldering, Lawrence Berkeley National Lab. (United States); A. Hoffmann, M. Kowalski, D. Küsters, K. Reif, M. Rigault, Univ. Bonn (Germany)

9147 4S Boresight calibration of FIFI-LS: in theory, in the lab and on sky [9147-181]

S. Colditz, Univ. Stuttgart (Germany); R. Klein, SOFIA-USRA, NASA Ames Research Ctr. (United States); S. Beckmann, Univ. Stuttgart (Germany) and Deutsches SOFIA Institut (Germany); A. Bryant, C. Fischer, F. Fumi, Univ. Stuttgart (Germany); N. Geis, Max-PlanckInstitut für extraterrestrische Physik (Germany); R. Hönle, Univ. Stuttgart (Germany);

A. Krabbe, Univ. Stuttgart (Germany) and Deutsches SOFIA Institut (Germany); L. W. Looney, Univ. of Illinois at Urbana-Champaign (United States); A. Poglitsch, W. Raab, Max-Planck-Institut für extraterrestrische Physik (Germany); S. E. Ragan, Max-Planck-Institut für Astronomie (Germany); F. Rebell, Univ. Stuttgart (Germany); M. L. Savage, SOFIA-USRA, NASA Ames Research Ctr. (United States)

$91474 \mathrm{~T}$ SPHERE/IRDIS: final performance assessment of the dual-band imaging and long slit spectroscopy modes [9147-182]

A. Vigan, Aix Marseille Univ., CNRS, Lab. d'Astrophysique de Marseille (France); M. Langlois, Ctr. de Recherche Astronomique de Lyon, CNRS, Univ. Lyon 1 (France); K. Dohlen, A. Zurlo, Aix Marseille Univ., CNRS, Lab. d'Astrophysique de Marseille (France); C. Moutou, Aix Marseille Univ., CNRS, Lab. d'Astrophysique de Marseille (France) and Canada-FranceHawaii Telescope (United States); A. Costille, C. Gry, F. Madec, D. Le Mignant, Aix Marseille Univ., CNRS, Lab. d'Astrophysique de Marseille (France); L. Gluck, Univ. Joseph FourierGrenoble 1, CNRS, Institut de Planétologie et d'Astrophysique de Grenoble (France); J.-F. Sauvage, ONERA (France)

$91474 \mathrm{U}$ Characterization of the atmospheric dispersion corrector of the Gemini planet imager [9147-183]

P. Hibon, Gemini South Observatory (Chile); S. Thomas, NASA Ames Research Ctr. (United States); J. Dunn, J. Atwood, L. Saddlemyer, NRC - Herzburg Institute of Astrophysics (Canada); N. Sadakuni, Gemini South Observatory (Chile); S. Goodsell, Gemini North Observatory (United States); B. Macintosh, Stanford Univ. (United States); J. Graham, Univ. of California, Berkeley (United States); M. Perrin, Space Telescope Science Institute (United 
States); F. Rantakyro, V. Fesquet, A. Serio, C. Quiroz, A. Cardwell, G. Gausachs, Gemini South Observatory (Chile); D. Savransky, Cornell Univ. (United States); D. Kerley, NRC Herzberg Institute of Astrophysics (Canada); M. Hartung, R. Galvez, K. Hardie, Gemini South Observatory (Chile)

$91474 \mathrm{~W} \quad$ Vibration specifications for VLT instruments [9147-185]

G. Jakob, J.-L. Lizon, European Southern Observatory (Germany)

9147 4X ARDOLORES: an Arduino based motors control system for DOLORES [9147-186] M. Gonzalez, H. Ventura, J. San Juan, L. Di Fabrizio, Telescopio Nazionale Galileo (Spain)

$91474 \mathrm{Y}$ Design and integration of a mechanism for focusing and alignment of the Echelle spectrograph for the telescope of $\mathbf{2 . 1}$ meters of the National Astronomic Observatory [9147-188]

H. O. A. Gutiérrez, A. S. Farah, J. M. Echevarria R., G. D. Sierra, Univ. Nacional Autónoma de México (Mexico)

$91474 Z$ Gemini planet imager observational calibrations IX: least-squares inversion flux extraction [9147-189]

Z. H. Draper, Univ. of Victoria (Canada) and NRC - Herzberg Institute of Astrophysics (Canada); C. Marois, NRC - Herzberg Institute of Astrophysics (Canada) and Univ. of Victoria (Canada); S. Wolff, Johns Hopkins Univ. (United States) and Space Telescope Science Institute (United States); M. Perrin, Space Telescope Science Institute (United States); P. J. Ingraham, Kavli Institute for Particle Astrophysics and Cosmology, Stanford Univ. (United States) and Univ. de Montréal (Canada); J.-B. Ruffio, Institut Supérieur de l'Aéronautique et de l'Espace (France) and SETI Institute (United States); F. T. Rantakyrö, M. Hartung, S. J. Goodsell, Gemini Observatory (Chile)

914750 Gemini planet imager one button approach [9147-190]

J. Dunn, D. Kerley, L. Saddlemyer, M. Smith, R. Wooff, NRC - Herzberg Institute of Astrophysics (Canada); D. Savransky, Cornell Univ. (United States); D. Palmer, B. Macintosh, Lawrence Livermore National Lab. (United States); J. Weiss, Univ. of California, Los Angeles (United States); C. Quiroz, F. T. Rantakyrö, Gemini Observatory (Chile); S. J. Goodsell, Gemini Observatory (United States)

914751 A format standard for efficient interchange of high-contrast direct imaging science products [9147-191]

É. Choquet, Space Telescope Science Institute (United States); A. Vigan, Aix Marseille Univ., CNRS, Lab. d'Astrophysique de Marseille (France); R. Soummer, Space Telescope Science Institute (United States); G. Chauvin, Univ. Joseph Fourier-Grenoble 1, CNRS, INSU, IPAG (France); L. Pueyo, M. D. Perrin, D. C. Hines, Space Telescope Science Institute (United States)

914752 Environmental control system for Habitable-zone Planet Finder (HPF) [9147-192] F. Hearty, The Pennsylvania State Univ. (United States) and Univ. of Virginia (United States); E. Levi, The Pennsylvania State Univ. (United States); M. Nelson, Univ. of Virginia (United States); S. Mahadevan, The Pennsylvania State Univ. (United States) and Ctr. for Exoplanets and Habitable Worlds (United States); A. Burton, Univ. of Virginia (United States); L. Ramsey, C. Bender, R. Terrien, S. Halverson, P. Robertson, A. Roy, The Pennsylvania State Univ. (United States) and Ctr. for Exoplanets and Habitable Worlds (United States); B. Blank, K. Blanchard, PulseRay (United States); G. Stefansson, The Pennsylvania State Univ. (United States) 
914753 Concept study for DREAMS: a Dedicated Robotic EArths-finding single-Mode Spectrograph [9147-193]

J. F. P. Spronck, Leiden Observatory, Leiden Univ. (Netherlands)

914754 Characterizing U-Ne hollow cathode lamps at near-IR wavelengths for the CARMENES survey [9147-194]

L. F. Sarmiento, A. Reiners, U. Seemann, U. Lemke, Institut für Astrophysik Göttingen

(Germany); J. Winkler, M. Pluto, E. W. Günther, Thüringer Landessternwarte Tautenburg (Germany); A. Quirrenbach, Landessternwarte, Zentrum für Astronomie der Univ.

Heidelberg (Germany); P. J. Amado, Instituto de Astrofisica de Andalucía (Spain); I. Ribas, Institut de Ciències de l'Espai (Spain); J. A. Caballero, Ctr. de Astrobiología (Spain); R. Mundt, Max-Planck-Institut für Astronomie (Germany); W. Seifert, Landessternwarte, Zentrum für Astronomie der Univ. Heidelberg (Germany)

914755 Gemini planet imager observational calibrations VIII: characterization and role of satellite spots [9147-195]

J. J. Wang, Univ. of California, Berkeley (United States); A. Rajan, Arizona State Univ. (United

States); J. R. Graham, Univ. of California, Berkeley (United States); D. Savransky, Cornell Univ. (United States); P. J. Ingraham, Kavli Institute for Particle Astrophysics and Cosmology, Stanford Univ. (United States); K. Ward-Duong, J. Patience, Arizona State Univ. (United States); R. J. De Rosa, Arizona State Univ. (United States) and Univ. of Exeter (United Kingdom); J. Bulger, Arizona State Univ. (United States); A. Sivaramakrishnan, M. D. Perrin, Space Telescope Science Institute (United States); S. J. Thomas, NASA Ames Research Ctr. (United States) and Univ. of California, Santa Cruz (United States); N. Sadakuni, Gemini Observatory (Chile); A. Z. Greenbaum, Johns Hopkins Univ. (United States) and Space Telescope Science Institute (United States); L. Pueyo, Space Telescope Science Institute (United States); C. Marois, NRC - Herzberg Institute of Astrophysics (Canada) and Univ. of Victoria (Canada); B. R. Oppenheimer, American Museum of Natural History (United States); P. Kalas, Univ. of California, Berkeley (United States); A. Cardwell, S. Goodsell, P. Hibon, F. T. Rantakyrö, Gemini Observatory (Chile)

914756 MASCARA: opto-mechanical design and integration [9147-196]

J. F. P. Spronck, A.-L. Lesage, R. Stuik, Leiden Observatory, Leiden Univ. (Netherlands); F. Bettonvil, Leiden Observatory, Leiden Univ. (Netherlands) and NOVA (Netherlands); I. A. G. Snellen, Leiden Observatory, Leiden Univ. (Netherlands)

914757 Study on a multi-delay spectral interferometry for stellar radial velocity measurement [9147-197]

K. Zhang, H. Jiang, J. Tang, H. Ji, Y. Zhu, Nanjing Institute of Astronomical Optics and Technology (China); L. Wang, National Astronomical Observatories (China)

914758 Test of multi-object exoplanet search spectral interferometer [9147-198]

K. Zhang, Nanjing Institute of Astronomical Optics and Technology (China); L. Wang, National Astronomical Observatories (China); H. Jiang, Y. Zhu, Y. Hou, S. Dai, J. Tang, Z. Tang, Y. Zeng, Y. Chen, L. Wang, Z. Hu, Nanjing Institute of Astronomical Optics and Technology (China)

9147 5A Diffraction-limited lucky imaging with a 12" commercial telescope [9147-201]

B. J. Baptista, National Geospatial-Intelligence Agency (United States) 
9147 5B Archon: a modern controller for high performance astronomical CCDs [9147-202] G. Bredthaver, Semiconductor Technology Associates Inc. (United States)

91475C Near field modal noise reduction using annealed optical fiber [9147-203] S. N. Osterman, Johns Hopkins Univ. Applied Physics Lab. (United States) and Univ. of Colorado, Boulder (United States); G. G. Ycas, National Institute of Standards and Technology (United States); C. L. Donaldson, Southwest Research Institute (United States); S. A. Diddams, National Institute of Standards and Technology (United States) and Univ. of Colorado, Boulder (United States); S. Mahadevan, L. W. Ramsey, The Pennsylvania State Univ. (United States); P. P. Plavchan, NASA Exoplanet Science Institute (United States) and Missouri State Univ. (United States)

9147 5D Control and operation of the $1.6 \mathrm{~m}$ New Solar Telescope in Big Bear [9147-204] J. Varsik, C. Plymate, P. Goode, A. Kosovichev, W. Cao, R. Coulter, K. Ahn, N. Gorceix, S. Shumko, Big Bear Solar Observatory (United States)

9147 5F DigiCam: fully digital compact camera for SST-1M telescope [9147-206]

J. A. Aguilar, Univ. de Genève (Switzerland); W. Bilnik, AGH Univ. of Science and Technology (Poland); L. Bogacz, Jagiellonian Univ. (Poland); T. Bulik, Univ. of Warsaw (Poland); A. Christov, D. della Volpe, Univ. de Genève (Switzerland); M. Dyrda, Instytut Fizyki Jaqdrowej (Poland); A. Frankowski, Nicolaus Copernicus Astronomical Ctr. (Poland); M. Grudzinska, Univ. of Warsaw (Poland); J. Grygorczuk, Centrum Badań Kosmicznych (Poland); M. Heller, Univ. de Genève (Poland); B. Idźkowski, Jagiellonian Univ. (Poland); M. Janiak, Nicolaus Copernicus Astronomical Ctr. (Poland); M. Jamrozy, Jagiellonian Univ. (Poland); M. Karczewski, Centrum Badań Kosmicznych (Poland); J. Kasperek, AGH Univ. of Science and Technology (Poland); E. Lyard, Univ. de Genève (Switzerland); A. Marszałek, Jagiellonian Univ. (Poland); J. Michałowski, Instyłut Fizyki Jąrowej (Poland); R. Moderski, Nicolaus Copernicus Astronomical Ctr. (Poland); T. Montaruli, A. Neronov, Univ. de Genève (Switzerland); J. Nicolau-Kukliński, Centrum Badań Kosmicznych (Poland); J. Niemiec, Instytut Fizyki Jądrowej (Poland); M. Ostrowski, Jagiellonian Univ. (Poland); P. Paśko, Ł. Płatos, Centrum Badań Kosmicznych (Poland); E. Prandini, Univ. de Genève (Switzerland); R. Pruchniewicz, AGH Univ. of Science and Technology (Poland); J. Rafalski, Instytut Fizyki Jadrowej (Poland); P. J. Rajda, AGH Univ. of Science and Technology (Poland); M. Rameez, Univ. de Genève (Switzerland); M. Rataj, Centrum Badań Kosmicznych (Poland); M. Rupiński, AGH Univ. of Science and Technology (Poland); K. Rutkowski, K. Seweryn, M. Sidz, Centrum Badań Kosmicznych (Poland); Ł. Stawarz, M. Stodulska, Jagiellonian Univ. (Poland); M. Stodulski, Instytut Fizyki Jądrowej (Poland); M. Tokarz, Centrum Badań Kosmicznych (Poland); S. Toscano, I. Troyano Pujadas, R. Walter, Univ. de Genève (Switzerland); P. Wawer, R. Wawrzaszek, L. Wiśniewski, Centrum Badań Kosmicznych (Poland); K. Ziętara, Jagiellonian Univ. (Poland); P. Ziółkowski, P. Żychowski, Instytut Fizyki Jądrowej (Poland)

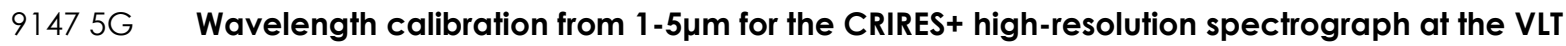
[9147-208]

U. Seemann, G. Anglada-Escude, Georg-August-Univ. Göttingen (Germany); D. Baade, P. Bristow, R. J. Dorn, European Southern Observatory (Germany); R. Follert, Thüringer Landessternwarte Tautenburg (Germany); D. Gojak, J. Grunhut, European Southern Observatory (Germany); A. P. Hatzes, Thüringer Landessternwarte Tautenburg (Germany); U. Heiter, Uppsala Univ. (Sweden); D. J. Ives, European Southern Observatory (Germany); P. Jeep, Georg-August Univ. Göttingen (Germany); Y. Jung, H.-U. Käufl, F. Kerber, B. Klein, J. Lizon, European Southern Observatory (Germany); M. Lockhart, Uppsala Univ. (Sweden); T. Löwinger, Thüringer Landessternwarte Tautenburg (Germany); T. Marquart, Uppsala Univ.

xxxviii 
(Sweden); E. Oliva, INAF - Osservatorio Astrofisico di Arcetri (Italy); J. Paufique, European Southern Observatory (Germany); N. Piskunov, Uppsala Univ. (Sweden); E. Pozna, European Southern Observatory (Germany); A. Reiners, Georg-August-Univ. Göttingen (Germany); A. Smette, J. Smoker, European Southern Observatory (Germany); E. Stempels, Uppsala Univ. (Sweden); E. Valenti, European Southern Observatory (Germany)

$91475 \mathrm{H}$ Characterization of new components for a miniaturized heterodyne infrared spectrometer [9147-209]

P. Krause, Univ. zu Köln (Germany); G. Sonnabend, RPG Radiometer Physics GmbH (Germany); L. Labadie, M. Sornig, Univ. zu Köln (Germany); R. Thomson, A. Arriola, HeriotWatt Univ. (United Kingdom); A. Rodenas, Univ. Rovira i Virgili (Spain); T. Hewagama, Univ. of Maryland, College Park (United States); F. Rutz, Fraunhofer-Institut für Angewandte Festkörperphysik (Germany)

9147 5K MUSE from Europe to the Chilean Sky [9147-362]

P. Caillier, Ctr. de Recherche Astrophysique de Lyon, CNRS, Univ. Claude-Bernard Lyon I (France); M. Accardo, European Southern Observatory (Germany); L. Adjali, Ctr. de Recherche Astrophysique de Lyon, CNRS, Univ. Claude-Bernard Lyon I (France); H. Anwand, Georg-August-Univ. Göttingen (Germany); R. Bacon, D. Boudon, L. Capoani, E. Daguisé, Ctr. de Recherche Astrophysique de Lyon, CNRS, Univ. Claude-Bernard Lyon I (France); M. Dupieux, Institut de Recherche en Astrophysique et Planétologie, CNRS, Univ. Paul Sabatier (France) and Observatoire Midi Pyrénées (France); C. Dupuy, European Southern Observatory (Germany); M. François, Ctr. de Recherche Astrophysique de Lyon, CNRS, Univ. Claude-Bernard Lyon I (France); A. Glindemann, D. Gojak, European Southern Observatory (Germany); F. Gonté, N. Haddad, European Southern Observatory (Chile); G. Hansali, Ctr. de Recherche Astrophysique de Lyon, CNRS, Univ. Claude-Bernard Lyon I (France); T. Hahn, Leibniz-Institut für Astrophysik Potsdam (Germany); A. Jarno, Ctr. de Recherche Astrophysique de Lyon, CNRS, Univ. Claude-Bernard Lyon I (France); A. Kelz, Leibniz-Institut für Astrophysik Potsdam (Germany); K. Koehler, Georg-August-Univ. Göttingen (Germany); J. Kosmalski, F. Laurent, Ctr. de Recherche Astrophysique de Lyon, CNRS, Univ. Claude-Bernard Lyon I (France); M. Larrieu, Institut de Recherche en Astrophysique et Planétologie, CNRS, Univ. Paul Sabatier (France) and Observatoire Midi Pyrénées (France); J. Lizon, European Southern Observatory (Germany); M. Loupias, Ctr. de Recherche Astrophysique de Lyon, CNRS, Univ. Claude-Bernard Lyon I (France); A. Manescau, European Southern Observatory (Germany); J. E. Migniau, Ctr. de Recherche Astrophysique de Lyon, CNRS, Univ. Claude-Bernard Lyon I (France); C. Monstein, ETH Zurich (Switzerland); H. Nicklas, Georg-August-Univ. Göttingen (Germany); L. Parès, Institut de Recherche en Astrophysique et Planétologie, CNRS, Univ. Paul Sabatier (France) and Observatoire Midi Pyrénées (France); A. Pécontal-Rousset, L. Piqueras, Ctr. de Recherche Astrophysique de Lyon, CNRS, Univ. Claude-Bernard Lyon I (France); R. Reiss, European Southern Observatory (Germany); A. Remillieux, E. Renault, Ctr. de Recherche Astrophysique de Lyon, CNRS, Univ. Claude-Bernard Lyon I (France); G. Rupprecht, European Southern Observatory (Germany); O. Streicher, Leibniz-Institut für Astrophysik Potsdam (Germany); R. Stuik, Leiden Observatory, Leiden Univ. (Netherlands); H. Valentin, Institut de Recherche en Astrophysique et Planétologie, CNRS, Univ. Paul Sabatier (France) and Observatoire Midi Pyrénées (France); J. Vernet, European Southern Observatory (Germany); P. Weilbacher, Leibniz-Institut für Astrophysik Potsdam (Germany); G. Zins, Institut de Planétologie et d'Astrophysique de Grenoble, CNRS, Univ. de Grenoble 1 (France) 
$91475 \mathrm{~L} \quad$ Spectrophotometric calibration of the Swope and duPont telescopes for the Carnegie supernova project 2 [9147-370]

J-P. Rheault, N. P. Mondrik, D. L. DePoy, J. L. Marshall, N. B. Suntzeff, Texas A\&M Univ. (United States)

\section{POSTER SESSION: SURVEY AND HIGH MULTIPLEX INSTRUMENTS}

$91475 \mathrm{M} \quad$ Fibre system of DESI [9147-210]

J. Allington-Smith, Durham Univ. (United Kingdom); C. Poppett, Lawrence Berkeley National Lab. (United States); D. Bramall, Durham Univ. (United Kingdom); J. Edelstein, Univ. of California, Berkeley (United States); R. Lafever, Lawrence Berkeley National Lab. (United States); J. Schmoll, C. Dunlop, G. Murray, Durham Univ. (United Kingdom); R. Besuner, Univ. of California, Berkeley (United States); G. Talbot, Durham Univ. (United Kingdom)

$91475 \mathrm{~N} \quad$ MEGARA fiber bundles [9147-211]

A. Pérez-Calpena, M. L. García-Vargas, FRACTAL S.L.N.E (Spain); X. Arrillaga, AVS Added Value Solutions (Spain); A. Gil de Paz, Univ. Complutense de Madrid (Spain); E. Sánchez-Blanco, I. Martínez-Delgado, FRACTAL S.L.N.E (Spain); M. A. Carrera, AVS Added Value Solutions (Spain); J. Gallego, Univ. Complutense de Madrid (Spain); E. Carrasco, Instituto Nacional de Astrofísica, Óptica y Electrónica (Mexico); F. M. Sánchez-Moreno, Univ. Politécnica de Madrid (Spain); J. Iglesias-Páramo, Instituto de Astrofísica de Andalucía (Spain)

91475Q Prime focus instrument of prime focus spectrograph for Subaru telescope [9147-213] S.-Y. Wang, Institute of Astronomy and Astrophysics, Academia Sinica (Taiwan); D. F. Braun, M. A. Schwochert, Jet Propulsion Lab. (United States); P.-J. Huang, Institute of Astronomy and Astrophysics, Academia Sinica (Taiwan); M. Kimura, Institute of Astronomy and Astrophysics, Academia Sinica (Taiwan) and Kavli Institute for the Physics and Mathematics of the Universe, The Univ. of Tokyo (Japan); H.-Y. Chen, Institute of Astronomy and Astrophysics, Academia Sinica (Taiwan); D. J. Reiley, P. Mao, California Institute of Technology (United States); C. D. Fisher, Jet Propulsion Lab. (United States); N. Tamura, Kavli Institute for the Physics and Mathematics of the Universe, The Univ. of Tokyo (Japan); Y.-C. Chang, Y.-S. Hu, H.-H. Ling, C.-Y. Wen, R. C.-Y. Chou, Institute of Astronomy and Astrophysics, Academia Sinica (Taiwan); N. Takato, Subaru Telescope, National Astronomical Observatory of Japan (United States); H. Sugai, Kavli Institute for the Physics and Mathematics of the Universe, The Univ. of Tokyo (Japan); Y. Ohyama, Institute of Astronomy and Astrophysics, Academia Sinica (Taiwan); H. Karoji, A. Shimono, Kavli Institute for the Physics and Mathematics of the Universe, The Univ. of Tokyo (Japan); A. Ueda, National Astronomical Observatory of Japan (Japan)

91475R MEGARA main optics opto-mechanics [9147-214] E. Castillo-Domínguez, J. L. Avilés, E. Carrasco, Instituto Nacional de Astrofísica, Óptica y Electrónica (Mexico); M. Maldonado, FRACTAL S.L.N.E (Spain); A. Gil de Paz, J. Gallego, Univ. Complutense de Madrid (Spain); R. Cedazo, Univ. Politécnica de Madrid (Spain); J. Iglesias, Instituto de Astrofísica de Andalucía (Spain)

$91475 \mathrm{~S}$ Metrology camera system of prime focus spectrograph for Subaru telescope [9147-215] S.-Y. Wang, R. C.-Y. Chou, Y.-C. Chang, P.-J. Huang, Y.-S. Hu, H.-Y. Chen, Institute of Astronomy and Astrophysics, Academia Sinica (Taiwan); N. Tamura, Kavli Institute for the Physics and Mathematics of the Universe, The Univ. of Tokyo (Japan); N. Takato, Subaru Telescope, National Astronomical Observatory of Japan (United States); H.-H. Ling, Institute 
of Astronomy and Astrophysics, Academia Sinica (Taiwan); J. E. Gunn, Princeton Univ. (United States); J. Karr, C.-H. Yan, Institute of Astronomy and Astrophysics, Academia Sinica (Taiwan); P. Mao, California Institute of Technology (United States); Y. Ohyama, Institute of Astronomy and Astrophysics, Academia Sinica (Taiwan); H. Karoji, H. Sugai, A. Shimono, Kavli Institute for the Physics and Mathematics of the Universe, The Univ. of Tokyo (Japan);

$91475 \mathrm{U} \quad$ Multiplexed astronomical images: advantages, method, and prototype instrument [9147-217]

S. Ben-Ami, B. Zackay, A. Rubin, I. Sagiv, A. Gal-Yam, E. O. Ofek, Weizmann Institute of Science (Israel)

9147 5V BOMBOLO: A 3-arms optical imager for SOAR Observatory [9147-218]

D. Guzmán, Pontificia Univ. Católica de Chile (Chile); R. Angeloni, Pontificia Univ. Católica de Chile (Chile) and Max-Planck-Institut fur Astronomie (Germany); T. Puzia, Pontificia Univ. Católica de Chile (Chile); D. Jones, Prime Optics (Australia); A. Jordán, Pontificia Univ. Católica de Chile (Chile); T. Anguita, Univ. Andrés Bello (Chile); S. Benecchi, Planetary Science Institute (United States) and Carnegie Institution of Washington (United States); E. Garcés, Pontificia Univ. Católica de Chile (Chile)

9147 5W MMP: multi mini prism device for ESPRESSO APSU, prototyping, and integration [9147-219] S. dell'Agostino, M. Riva, M. Genoni, M. Landoni, M. Moschetti, M. Aliverti, INAF Osservatorio Astronomico di Brera (Italy); F. Pepe, D. Mégevand, Observatoire de Genève (Switzerland); F. M. Zerbi, INAF - Osservatorio Astronomico di Brera (Italy); S. Cristiani, INAF Osservatorio Astronomico di Trieste (Italy); A. Cabral, Univ. de Lisboa (Portugal);

P. Conconi, INAF - Osservatorio Astronomico di Brera (Italy)

$91475 Z$ Integration and test activities for the SUMIRE prime focus spectrograph at LAM [9147-222] F. Madec, J. Marc, S. Pascal, A. Bozier, D. Le Mignant, S. Vives, D. Ferrand, T. Pegot-Ogier, G. Arthaud, Aix Marseille Univ., CNRS, Lab. d'Astrophysique de Marseille (France); M. Golebiowski, Johns Hopkins Univ. (United States); H. Sugai, N. Tamura, Kavli Institute for the Physics and Mathematics of the Universe, The Univ. of Tokyo (Japan); J. Gunn, Princeton Univ. (United States); S. Smee, Johns Hopkins Univ. (United States); L. Oliveira, Lab. Nacional de Astrofísica (Brazil)

914760 Efficient and affordable catadioptric spectrograph designs for 4MOST and Hector [9147-223]

W. Saunders, Australian Astronomical Observatory (Australia)

914762 Current status of the spectrograph system for the SuMIRe/PFS [9147-227]

S. Vives, D. Le Mignant, Aix Marseille Univ., CNRS, Lab. d'Astrophysique de Marseille (France); J. E. Gunn, Princeton Univ. (United States); S. Smee, Johns Hopkins Univ. (United States); L. Souza de Oliveira, Lab. Nacional de Astrofísica (Brazil); N. Tamura, H. Sugai, Institute for the Physics and Mathematics of the Universe, The Univ. of Tokyo (Japan); R. Barkhouser, Johns Hopkins Univ. (United States); A. Bozier, Aix Marseille Univ., CNRS, Lab. d'Astrophysique de Marseille (France); M. A. Carr, Princeton Univ. (United States); A. C. de Oliveira, Lab. Nacional de Astrofísica (Brazil); D. Ferrand, Aix Marseille Univ., CNRS, Lab. d'Astrophysique de Marseille (France); M. Golebiowski, M. Hart, S. Hope, Johns Hopkins Univ. (United States); M. Jaquet, F. Madec, S. Pascal, T. Pegot-Ogier, Aix Marseille Univ., CNRS, Lab. d'Astrophysique de Marseille (France); M. Vital de Arruda, Lab. Nacional de Astrofísica (Brazil) 
914763 Focal ratio degradation performance of fiber positioning technology used in the Dark Energy Spectroscopic Instrument (DESI) [9147-228]

C. L. Poppett, Lawrence Berkeley National Lab. (United States); J. Edelstein, R. Besuner, Space Sciences Lab. (United States); J. H. Silber, Lawrence Berkeley National Lab. (United States)

914764 Virtual MOONS: a focal plane simulator for the MOONS thousand-fiber NIR spectrograph [9147-229]

G. Li Causi, INAF - Osservatorio Astronomico di Roma (Italy); A. Cabral, Univ. of Lisbon (Portugal); D. Ferruzzi, INAF - Osservatorio Astrofisico di Arcetri (Italy); G. Finger, European Southern Observatory (Germany); G. Giacalone, Univ. di Roma La Sapienza (Italy);

I. Guinouard, GEPI, Observatoire de Paris, CNRS, Univ. Paris Diderot (France); D. Lorenzetti, INAF - Osservatorio Astronomico di Roma (Italy); E. Oliva, INAF - Osservatorio Astrofisico di Arcetri (Italy); F. Pedichini, INAF - Osservatorio Astronomico di Roma (Italy); F. Royer, GEPI, Observatoire de Paris, CNRS, Univ. Paris Diderot (France); S. Todd, STFC UK Astronomy Technology Ctr. (United Kingdom); F. Vitali, INAF - Osservatorio Astronomico di Roma (Italy)

914765 Design and performance of a F/\#-conversion microlens for prime focus spectrograph at Subaru Telescope [9147-230]

N. Takato, Y. Tanaka, Subaru Telescope, National Astronomical Observatory of Japan (United States); J. E. Gunn, Princeton Univ. (United States); N. Tamura, A. Shimono, H. Sugai, H. Karoji, Kavli Institute for the Physics and Mathematics of the Universe, The Univ. of Tokyo (Japan); A. Ueda, K. Waseda, National Astronomical Observatory of Japan (Japan); M. Kimura, Y. Ohyama, Institute of Astronomy and Astrophysics, Academia Sinica (Taiwan)

914766 Preliminary results on the characterization and performances of ZBLAN fiber for infrared spectrographs [9147-231]

M. Iuzzolino, A. Tozzi, N. Sanna, L. Zangrilli, E. Oliva, INAF - Osservatorio Astrofisico di Arcetri (Italy)

914767 The hardware control system for WEAVE at the William Herschel telescope [9147-232] J. M. Delgado Hernandez, L. F. Rodríguez-Ramos, Instituto de Astrofísica de Canarias (Spain); D. Cano Infantes, C. Martin, C. Bevil, S. Picó, K. M. Dee, D. C. Abrams, Isaac Newton Group of Telescopes (Spain); I. J. Lewis, Univ. of Oxford (United Kingdom); J. Pragt, R. Stuik, N. Tromp, NOVA ASTRON (Netherlands); G. Dalton, Univ. of Oxford (United Kingdom); J. A. L. Aguerri, Univ. de La Laguna (Spain); P. Bonifacio, Observatoire de Paris à Meudon (France); K. F. Middleton, Rutherford Appleton Lab. (United Kingdom);

S. C. Trager, Univ. of Groningen (Netherlands)

\section{Part Four}

$91476 \mathrm{~A} \quad$ 4MOST fiber feed concept design [9147-235]

D. M. Haynes, R. Winkler, A. Saviauk, R. Haynes, S. Barden, O. Bellido-Tirado, S. Baver, R. S. de Jong, Leibniz-Institut für Astrophysik Potsdam (Germany); E. Depagne, South African Astronomical Observatory (South Africa); F. Dionies, K. Ehrlich, A. Kelz, Leibniz-Institut für Astrophysik Potsdam (Germany); W. Saunders, Australian Astronomical Observatory (Australia); M. Woche, Leibniz-Institut für Astrophysik Potsdam (Germany) 
9147 6B Scrambling and modal noise mitigation in the Habitable Zone Planet Finder fiber feed [91 47-236]

A. Roy, S. Halverson, S. Mahadevan, L. W. Ramsey, The Pennsylvania State Univ. (United States)

9147 6C Development of a new readout system for the near-infrared detector of HONIR [9147-237] T. Ui, Hiroshima Univ. (Japan); S. Sako, The Univ. of Tokyo (Japan); T. Yamashita, National Astronomical Observatory of Japan (Japan); H. Akitaya, K. S. Kawabata, Hiroshima Univ. (Japan); H. Nakaya, National Astronomical Observatory of Japan (Japan); Y. Moritani, R. Itoh, K. Takaki, T. Urano, I. Ueno, T. Ohsugi, M. Yoshida, Hiroshima Univ. (Japan); H. Nakao, Hokkaido Univ. (Japan); Y. Hashiba, The Univ. of Tokyo (Japan)

9147 6D Okayama astrophysical observatory wide field camera [9147-238]

K. Yanagisawa, Y. Shimizu, K. Okita, D. Kuroda, H. Koyano, H. Tsutsui, H. Toda, H. Izumiura, National Astronomical Observatory of Japan (Japan); M. Yoshida, Hiroshima Univ. (Japan); K. Ohta, Kyoto Univ. (Japan); N. Kawai, Tokyo Institute of Technology (Japan); T. Yamamuro, OptCraft (Japan)

$91476 \mathrm{E} \quad$ Polarization properties of a birefringent fiber optic image slicer for diffraction-limited dualbeam spectropolarimetry [9147-239]

T. Schad, H. Lin, Institute for Astronomy, Univ. of Hawai'i (United States); K. Ichimoto, Kyoto Univ. (Japan); Y. Katsukawa, National Astronomical Observatory of Japan (Japan)

$91476 \mathrm{~F}$ The S4I prototype, a beam-slicer dedicated to the new generation Multichannel Subtractive Double Pass for EST imaging spectropolarimetry [9147-240] F. Sayède, GEPI, Observatoire de Paris (France); P. Mein, J.-M. Malherbe, LESIA, Observatoire de Paris (France); J.-P. Amans, GEPI, Observatoire de Paris (France); D. Crussaire, R. Lecocguen, LESIA, Observatoire de Paris (France)

$91476 \mathrm{H} \quad$ The design of the WEAVE spectrograph [9147-242]

K. Rogers, STFC Rutherford Appleton Lab. (United Kingdom); R. Stuik, NOVA ASTRON (Netherlands) and Leiden Observatory, Leiden Univ. (Netherlands); I. A. Steele, Liverpool John Moores Univ. (United Kingdom); J. Pragt, NOVA ASTRON (Netherlands);

K. F. Middleton, STFC Rutherford Appleton Lab. (United Kingdom); S. Bates, Liverpool John Moores Univ. (United Kingdom); J. Kragt, N. Tromp, D. Lesman, NOVA ASTRON (Netherlands); E. Lhomé, Isaac Newton Group of Telescopes (Spain); G. Dalton, STFC Rutherford Appleton Lab. (United Kingdom) and Univ. of Oxford (United Kingdom); S. Trager, Univ. of Groningen (Netherlands); R. Navarro, NOVA ASTRON (Netherlands); D. C. Abrams, Isaac Newton Group of Telescopes (Spain); I. Tosh, STFC Rutherford Appleton Lab. (United Kingdom); A. Jasko, Konkoly Thege Miklós Astronomical Institute (Hungary); C. Martin, N. O'Mahony, S. Pico, D. Cano Infantes, Isaac Newton Group of Telescopes (Spain); A. Bianca, INAF - Osservatorio Astronomico di Brera (Italy); J. Delgado, L. F. Rodríguez, Instituto de Astrofísica de Canarias (Spain)

914761 The 4MOST instrument concept overview [9147-243]

R. Haynes, S. Barden, R. de Jong, O. Schnurr, O. Bellido, J. Walcher, D. Haynes, R. Winkler, S.-M. Baver, F. Dionies, A. Saviauk, C. Chiappini, A. Schwope, J. Brynnel, M. Steinmetz, Leibniz-Institut für Astrophysik Potsdam (Germany); R. McMahon, Univ. of Cambridge (United Kingdom); S. Feltzing, Lund Observatory (Sweden); P. Francois, GEPI, Observatoire de Paris à Meudon (France); S. Trager, Kapteyn Astronomical Institute, Univ. of Groningen (Netherlands); I. Parry, M. Irwin, N. Walton, D. King, Univ. of Cambridge (United Kingdom); D. Sun, Cavendish Lab., Univ. of Cambridge (United Kingdom); 
E. Gonzalez-Solares, Univ. of Cambridge (United Kingdom); I. Tosh, Rutherford Appleton Lab. (United Kingdom); G. Dalton, Univ. of Oxford (United Kingdom); K. Middleton,

Rutherford Appleton Lab. (United Kingdom); P. Bonifacio, P. Jagourel, S. Mignot, M. Cohen, J.-P. Amans, F. Royer, P. Sartoretti, GEPI, Observatoire de Paris à Meudon (France); J. Pragt, G. Gerlofsma, R. Roelfsema, R. Navarro, ASTRON (Netherlands); G. Thimm, Univ. Heidelberg (Germany); W. Seifert, N. Christlieb, H. Mandel, Landessternwarte, Zentrum für Astronomie der Univ. Heidelberg (Germany); T. Trifonov, Univ. Heidelberg (Germany); W. Xu, Optical System Engineering (Germany);F. Lang-Bardl, B. Muschielok, J. Schlichter, H.-J. Hess, Univ.Sternwarte München (Germany); F. Grupp, H. Boehringer, T. Boller, T. Dwelly, R. Bender, Max-Planck-Institut für extraterrestrische Physik (Germany); P. Rosati, O. Iwert, G. Finger, J.-L. Lizon L'Allemand, European Southern Observatory (Germany); W. Saunders, A. Sheinis, G. Frost, T. Farrell, L. Waller, Australian Astronomical Observatory (Australia); E. Depagne, Leibniz-Institut für Astrophysik Potsdam (Germany) and South African Astronomical Observatory (South Africa); F. Laurent, P. Caillier, J. Kosmalski, J. Richard, R. Bacon, Ctr. de Recherche Astrohysique de Lyon (France); W. Ansorge, RAMS-CON (Germany)

9147 6K Development of a simultaneous two-color near-infrared multi-object spectrograph SWIMS for the TAO 6.5-m telescope [9147-245]

K. Motohara, M. Konishi, H. Takahashi, K. Tateuchi, Y. Kitagawa, S. Todo, N. M. Kato, R. Ohsawa, The Univ. of Tokyo (Japan); T. Aoki, Kiso Observatory, The Univ. of Tokyo (Japan); K. Asano, M. Doi, T. Kamizuka, K. Kawara, K. Kohno, The Univ. of Tokyo (Japan); S. Koshida, The Univ. of Tokyo (Japan) and Pontificia Univ. Católica de Chile (Chile); T. Minezaki, T. Miyata, T. Morokuma, K. Okada, S. Sako, The Univ. of Tokyo (Japan); T. Soyano, Kiso Observatory, The Univ. of Tokyo (Japan); Y. Tamura, T. Tanabe, M. Tanaka, The Univ. of Tokyo (Japan); K. Tarusawa, Kiso Observatory, The Univ. of Tokyo (Japan); M. Uchiyama, Y. Yoshii, The Univ. of Tokyo (Japan)

$91476 \mathrm{~L} \quad$ Sky background subtraction with fiber-fed spectrographs [9147-246] M. Puech, GEPI, Observatoire de Paris, CNRS, Univ. Paris Diderot (France); M. Rodrigues, GEPI, Observatoire de Paris, CNRS, Univ. Paris Diderot (France) and European Southern Observatory (Chile); Y. Yang, H. Flores, F. Royer, K. Disseau, GEPI, Observatoire de Paris, CNRS, Univ. Paris Diderot (France); T. Gonçalves, Univ. Federal do Rio de Janeiro (Brazil); F. Hammer, GEPI, Observatoire de Paris, CNRS, Univ. Paris Diderot (France); M. Cirasuolo, SUPA, Institute for Astronomy, Univ. of Edinburgh, Royal Observatory (United Kingdom); C. J. Evans, UK Astronomy Technology Ctr. (United Kingdom); G. Li Causi, INAF Osservatorio Astronomico di Roma (Italy); R. Maiolino, Univ. of Cambridge (United Kingdom); C. Melo, European Southern Observatory (Chile)

$91476 \mathrm{M}$ Accuracy research for survey telescope fiber position measurement [9147-247] Z. Zhou, Z. Liu, H. Hu, J. Wang, C. Zhai, J. Chu, Univ. of Science and Technology of China (China)

$91476 \mathrm{~N}$ Development of multi-object spectroscopy unit for simultaneous-color wide-field infrared multi-object spectrograph [9147-248]

H. Takahashi, M. Konishi, K. Motohara, N. M. Kato, K. Tateuchi, Y. Kitagawa, S. Todo, The Univ. of Tokyo (Japan)

9147 6P Achieving decameter velocity precision with a multi-object spectrograph [9147-251] J. I. Bailey III, M. L. Mateo, Univ. of Michigan (United States); J. D. Crane, Carnegie Observatories (United States) 
$91476 Q \quad$ Wide FastCam: a wide field imaging camera for the TCS [9147-252]

G. Murga, IDOM (Spain); A. Oscoz, R. López, Instituto de Astrofísica de Canarias (Spain);

R. Campo, U. Etxegarai, IDOM (Spain); E. Pallé, Instituto de Astrofísica de Canarias (Spain)

9147 6R The DESI wide field corrector optics [9147-253]

P. Doel, Univ. College London (United Kingdom); M. J. Sholl, Lawrence Berkeley National Lab. (United States); M. Liang, National Optical Astronomy Observatory (United States);

D. Brooks, Univ. College London (United Kingdom); B. Flaugher, G. Gutierrez, S. Kent, Fermi National Accelerator Lab. (United States); M. Lampton, T. Miller, Lawrence Berkeley National Lab. (United States); D. Sprayberry, National Optical Astronomy Observatory (United States)

$91476 S$ MEGARA cryostat advanced design [9147-254]

D. Ferrusca, E. Castillo-Domínguez, M. Velázquez, Instituto Nacional de Astrofísica, Óptica y Electrónica (Mexico); A. Gil de Paz, Univ. Complutense de Madrid (Spain); E. Carrasco, Instituto Nacional de Astrofísica, Óptica y Electrónica (Mexico); J. Gallego, Univ. Complutense de Madrid (Spain); R. Cedazo, Univ. Politécnica de Madrid (Spain); J. Iglesias-Páramo, Instituto de Astrofísica de Andalucia (Spain)

$91476 \mathrm{~T}$ Performance of the Southern African Large Telescope (SALT) High Resolution Spectrograph (HRS) [9147-256]

L. A. Crause, South African Astronomical Observatory (South Africa); R. M. Sharples,

D. G. Bramall, J. Schmoll, P. Clark, E. J. Younger, Durham Univ. (United Kingdom);

L. M. G. Tyas, SALT Foundation (South Africa); S. G. Ryan, Univ. of Hertfordshire

(United Kingdom); J. D. Brink, O. J. Strydom, D. A. Buckley, M. Wilkinson, SALT Foundation

(South Africa); S. M. Crawford, Southern African Large Telescope (South Africa);

É. Depagne, SALT Foundation (South Africa)

$91476 \mathrm{U}$ VIRUS instrument enclosures [9147-257]

T. Prochaska, R. Allen, N. Mondrik, J. P. Rheault, M. Sauseda, E. Boster, M. James, M. Rodriguez-Patino, G. Torres, J. Ham, E. Cook, D. Baker, D. L. DePoy, J. L. Marshall, Texas A\&M Univ. (United States); G. J. Hill, McDonald Observatory, The Univ. of Texas at Austin (United States) and The Univ. of Texas at Austin (United States); D. Perry, R. D. Savage, J. M. Good, B. L. Vattiat, McDonald Observatory, The Univ. of Texas at Austin (United States);

$91476 \mathrm{~V}$ Presenting a high accuracy Theta-Phi-style fiber-positioner prototype with a 15-mm pitch [9147-258]

M. Haeuser, F. Lang-Bardl, J. Richter, H.-J. Hess, A. Degwert, A. Karasz, R. Kosyra, Univ.-Sternwarte München (Germany); U. Hopp, R. Bender, Univ.-Sternwarte München (Germany) and Max-Planck-Institut für extraterrestrische Physik (Germany)

9147 6X ROS2: a multichannel vision for the robotic REM telescope [9147-260]

E. Molinari, INAF - Istituto di Astrofisica Spaziale e Fisica Cosmica (Italy) and INAF Fundación Galileo Galilei (Spain); S. Covino, G. Crimi, INAF - Osservatorio Astronomico di Brera (Italy); F. D'Alessio, INAF - Osservatorio Astronomico di Roma (Italy); S. Incorvaia, INAF - Istituto di Astrofisica Spaziale e Fisica Cosmica (Italy); D. Fugazza, INAF - Osservatorio Astronomico di Brera (Italy); P. Spanò, NRC - Herzburg Institute of Astrophysics (Canada); G. Toso, D. Tresoldi, INAF - Istituto di Astrofisica Spaziale e Fisica Cosmica (Italy); F. Vitali, INAF - Osservatorio Astronomico di Brera (Italy) 
$91476 \mathrm{Y}$ Towards a spectroscopic survey of one hundred thousand spatially resolved galaxies with Hector [9147-261]

J. S. Lawrence, Australian Astronomical Observatory (Australia); J. Bland-Hawthorn, The Univ. of Sydney (Australia); D. Brown, Australian Astronomical Observatory (Australia); J. J. Bryant, Australian Astronomical Observatory (Australia) and The Univ. of Sydney (Australia); G. Cecil, The Univ. of North Carolina (United States); R. Content, Australian Astronomical Observatory (Australia); S. Croom, The Univ. of Sydney (Australia); L. Gers, P. R. Gillingham, Australian Astronomical Observatory (Australia); S. Richards, The Univ. of Sydney (Australia); W. Saunders, N. Staszak, Australian Astronomical Observatory (Australia)

$91476 Z$ Monitoring the atmospheric throughput at Cerro Tololo Inter-American Observatory with aTmCam [9147-262]

T. Li, D. L. DePoy, J. L. Marshall, D. Q. Nagasawa, D. W. Carona, S. Boada, Texas A\&M Univ. (United States)

914770 Methods for the detection and the characterization of low mass companions using the IFS of SPHERE [9147-263]

A. Zurlo, Aix Marseille Univ., CNRS, Lab. d'Astrophysique de Marseille (France) and INAF Osservatorio Astronomico di Padova (Italy); D. Mesa, R. Gratton, R. Claudi, S. Desidera, E. Giro, INAF - Osservatorio Astronomico di Padova (Italy); J.-L. Beuzit, Univ. Joseph FourierGrenoble 1, CNRS-INSU, Institut de Planétologie et d'Astrophysique de Grenoble (France); K. Dohlen, Aix Marseille Univ., CNRS, Lab. d'Astrophysique de Marseille (France); D. Mouillet, P. Puget, Univ. Joseph Fourier-Grenoblel, CNRS-INSU, Institut de Planétologie et d'Astrophysique de Grenoble (France); F. Wildi, Observatoire de Genève, Univ. de Genève (Switzerland); M. Feldt, O. Moeller-Nilsson, Max-Planck-Institut für Astronomie (Germany); A. Baruffolo, D. Fantinel, B. Salasnich, INAF - Osservatorio Astronomico di Padova (Italy); M. Kasper, European Southern Observatory (Germany); A. Costille, Aix Marseille Univ., CNRS, Lab. d'Astrophysique de Marseille (France); J.-F. Sauvage, ONERA (France);

A. Vigan, C. Moutou, Aix Marseille Univ., CNRS, Lab. d'Astrophysique de Marseille (France); M. Langlois, Ctr. de Recherche Astronomique de Lyon, CNRS, Univ. Lyon 1 (France); J. Antichi, INAF - Osservatorio Astrofisico di Arcetri (Italy); A. Pavlov, Max-Planck-Institut für Astronomie (Germany); N. Zimmerman, European Southern Observatory (Germany); M. Turatto, INAF - Osservatorio Astronomico di Padova (Italy)

914771 MEGARA optical manufacturing process [9147-264]

E. Carrasco, Instituto Nacional de Astrofísica, Óptica y Electrónica (Mexico); G. Páez, Ctr. de Investigaciones en Óptica (Mexico); F. Granados, E. Percino, E. Castillo-Domínguez, J. L. Avilés, Instituto Nacional de Astrofísica, Óptica y Electrónica (Mexico); M. L. GarcíaVargas, Fractal S.L.N.E (Spain); A. Gil de Paz, J. Gallego, Univ. Complutense de Madrid (Spain); J. Iglesias-Páramo, Instituto de Astrofísica de Andalucía (Spain); R. Cedazo, Univ. Politécnica de Madrid (Spain)

914772 High speed wide field CMOS camera for Transneptunian Automatic Occultation Survey [9147-265]

S.-Y. Wang, Institute of Astronomy and Astrophysics, Academia Sinica (Taiwan);

J. C. Geary, S. M. Amato, Harvard-Smithsonian Ctr. for Astrophysics (United States); Y.-S. Hu,

H.-H. Ling, P.-J. Huang, Institute of Astronomy and Astrophysics, Academia Sinica (Taiwan);

G. Furesz, Harvard-Smithsonian Ctr. for Astrophysics (United States); H.-Y. Chen,

Y.-C. Chang, Institute of Astronomy and Astrophysics, Academia Sinica (Taiwan);

A. Szentgyorgyi, Harvard-Smithsonian Ctr. for Astrophysics (United States); M. Lehner, Institute of Astronomy and Astrophysics, Academia Sinica (Taiwan); T. Norton, HarvardSmithsonian Ctr. for Astrophysics (United States) 
914773 Final optical design for the WEAVE two-degree prime focus corrector [9147-266] T. Agócs, NOVA ASTRON (Netherlands); D. C. Abrams, E. Lhomé, K. Dee, Isaac Newton Group of Telescopes (Spain); G. Dalton, STFC Rutherford Appleton Lab. (United Kingdom) and Univ. of Oxford (United Kingdom); K. Middleton, STFC Rutherford Appleton Lab. (United Kingdom); P. Bonifacio, GEPI, Observatoire de Paris (France); J. A. L. Aguerri, Instituto de Astrofísica de Canarias (Spain); S. C. Trager, Univ. of Groningen (Netherlands)

914774 The guider and wavefront curvature sensor subsystem for the Large Synoptic Survey Telescope [9147-267]

V. J. Riot, Lawrence Livermore National Lab. (United States); K. Arndt, Purdue Univ. (United States); C. Claver, Large Synoptic Survey Telescope (United States); P. E. Doherty, Harvard Univ. (United States); D. K. Gilmore, P. A. Hascall, S. Herrmann, SLAC National Accelerator Lab. (United States); I. Kotov, P. O'Connor, Brookhaven National Lab. (United States); J. Sebag, Large Synoptic Survey Telescope (United States); C. W. Stubbs, Harvard Univ. (United States); M. Warner, Cerro Tololo Inter-American Observatory (Chile)

914775 VIRUS: assembly, testing and performance of 33,000 fibres for HETDEX [9147-269]

A. Kelz, T. Jahn, D. Haynes, Leibniz-Institut für Astrophysik Potsdam (Germany); G. J. Hill, H. Lee, J. D. Murphy, The Univ. of Texas at Austin (United States); J. Neumann, Leibniz-Institut für Astrophysik Potsdam (Germany); H. Nicklas, Georg-August-Univ. Göttingen (Germany); M. Rutowska, C. Sandin, O. Streicher, Leibniz-Institut für Astrophysik Potsdam (Germany); S. Tuttle, The Univ. of Texas at Austin (United States); M. Fabricius, Max-Planck-Institut für extraterrestrische Physik (Germany); S. M. Baver, Leibniz-Institut für Astrophysik Potsdam (Germany); B. Vattiat, The Univ. of Texas at Austin (United States); H. Anwand, GeorgAugust-Univ. Göttingen (Germany); R. Savage, The Univ. of Texas at Austin (United States)

914777 A multi-pixel room-temperature local oscillator subsystem for array receivers at $1.9 \mathrm{THz}$ [9147-272]

J. V. Siles, I. Mehdi, C. Lee, R. Lin, J. Kawamura, E. Schlecht, P. J. Bruneau, P. F. Goldsmith, Jet Propulsion Lab. (United States)

914778 Multi-object fibre spectroscopy at the WHT: performance enhancements of AF2+WYFFOS [9147-374]

L. Domínquez Palmero, D. Cano, C. Fariña, R. Bassom, E. Lhomé, N. O'Mahony, J. Skvarc,

A. Ridings, C. R. Benn, D. C. Abrams, M. Balcells, Isaac Newton Group of Telescopes (Spain)

914779 The Zwicky transient facility observing system [9147-375]

R. M. Smith, R. G. Dekany, Caltech Optical Observatories (United States); C. Bebek, Lawrence Berkeley National Lab. (United States); E. Bellm, K. Bui, J. Cromer, P. Gardner, Caltech Optical Observatories (United States); M. Hoff, Lawrence Berkeley National Lab. (United States); S. Kaye, S. Kulkarni, Caltech Optical Observatories (United States); A. Lambert, M. Levi, Lawrence Berkeley National Lab. (United States); D. Reiley, Caltech Optical Observatories (United States)

\section{POSTER SESSION: HIGH SPECTRAL AND SPATIAL RESOLUTION INSTRUMENTS}

9147 7B Gemini planet imager observational calibrations X: non-redundant masking on GPI [9147-135]

A. Z. Greenbaum, Johns Hopkins Univ. (United States); A. Cheetham, The Univ. of Sydney (Australia); A. Sivaramakrishnan, P. Tuthill, B. Norris, Space Telescope Science Institute (Australia); L. Pueyo, The Univ. of Sydney (United States); N. Sadakuni, F. Rantakyrö, 
P. Hibon, S. Goodsell, M. Hartung, A. Serio, A. Cardwell, Gemini Observatory (Chile); L. Poyneer, Lawrence Livermore National Lab. (United States); B. Macintosh, Lawrence Livermore National Lab. (United States) and Kavli Institute for Particle Astrophysics and Cosmology, Stanford Univ. (United States); D. Savransky, Cornell Univ. (United States); M. D. Perrin, The Univ. of Sydney (Australia); S. Wolff, Johns Hopkins Univ. (United States); P. Ingraham, Kavli Institute for Particle Astrophysics and Cosmology, Stanford Univ. (United States); S. Thomas, NASA Ames Research Ctr. (United States)

$91477 \mathrm{C}$ InnoPOL: an EMCCD imaging polarimeter and 85-element curvature AO system on the 3.6m AEOS telescope for cost effective polarimetric speckle suppression [91 47-274]

D. Harrington, Institute for Astronomy, Univ. of Hawai'i (United States) and KiepenheverInstitut für Sonnenphysik (Germany); S. Berdyugina, Kiepenheuer-Institut für Sonnenphysik (Germany); M. Chun, C. Ftaclas, Institute for Astronomy, Univ. of Hawai'i (United States); D. Gisler, Kiepenhever-Institut für Sonnenphysik (Germany); J. Kuhn, Institute for Astronomy, Univ. of Hawaili (United States)

9147 7D APSU @ ESPRESSO: final design towards the integration [9147-275] M. Riva, P. Conconi, M. Moschetti, S. Dell'Agostino, M. Genoni, M. Aliverti, INAF Osservatorio Astronomico di Brera (Italy); F. Pepe, D. Mégevand, Observatoire de Genève, Univ. de Genève (Switzerland); F. M. Zerbi, INAF - Osservatorio Astronomico di Brera (Italy); S. Cristiani, INAF - Osservatorio Astronomico di Trieste (Italy); A. Cabral, Univ. de Lisboa (Portugal); P. Spanò, NRC - Herzberg Institute de Astrophysics (Canada)

9147 7F Design of a radial velocity spectrograph for the Moletai Astronomical Observatory [9147-277]

C. A. Jurgenson, D. A. Fischer, T. M. McCracken, R. A. Stoll, A. E. Szymkowiak, M. J. Giguere, Yale Univ. (United States); F. G. Santoro, G. Muller, ASTRO Electro-Mechanical Engineering (United States)

$91477 G \quad$ ESPRESSO front end: modular opto-mechanical integration for astronomical instrumentation [9147-278]

M. Riva, M. Aliverti, M. Moschetti, M. Landoni, S. Dell'Agostino, INAF - Osservatorio Astronomico di Brera (Italy); F. Pepe, D. Mégevand, Observatoire de Genève, Univ. de Genève (Switzerland); F. M. Zerbi, INAF - Osservatorio Astronomico di Brera (Italy); S. Cristiani, INAF - Osservatorio Astronomico di Trieste (Italy); A. Cabral, Univ. de Lisboa (Portugal)

$91477 \mathrm{H}$ Gemini planet imager observational calibrations IV: wavelength calibration and flexure correction for the integral field spectograph [9147-279]

S. G. Wolff, Johns Hopkins Univ. (United States); M. D. Perrin, Space Telescope Science Institute (United States); J. Maire, Dunlap Institute for Astronomy and Astrophysics, Univ. of Toronto (Canada); P. J. Ingraham, Stanford Univ. (United States); F. T. Rantakyrö, P. Hibon, Gemini Observatory (Chile)

9147 7I RHEA: the ultra-compact replicable high-resolution exoplanet and asteroseismology spectrograph [9147-280]

T. Feger, C. Bacigalupo, Macquarie Univ. (Australia); T. R. Bedding, The Univ. of Sydney (Australia); J. Bento, D. W. Coutts, Macquarie Univ. (Australia); M. J. Ireland, Australian National Univ. (Australia); Q. A. Parker, Macquarie Univ. (Australia) and Australian Astronomical Observatory (Australia); A. Rizzuto, I. Spaleniak, Macquarie Univ. (Australia) 
$91477 \mathrm{~J}$ SHARK (System for coronagraphy with High order Adaptive optics from R to K band), a proposal for the LBT $2^{\text {nd }}$ generation instrumentation [9147-281]

J. Farinato, INAF - Osservatorio Astronomico di Padova (Italy); F. Pedichini, INAF -

Osservatorio Astronomico di Roma (Italy); E. Pinna, INAF - Osservatorio Astrofisico di Arcetri

(Italy); F. Bacciotti, C. Baffa, INAF - Osservatorio Astronomico di Arcetri (Italy); A. Baruffolo,

M. Bergomi, INAF - Osservatorio Astronomico di Padova (Italy); P. Bruno, INAF - Osservatorio Astrofisico di Catania (Italy); E. Cappellaro, INAF - Osservatorio Astronomico di Padova (Italy); L. Carbonaro, INAF - Osservatorio Astronomico di Arcetri (Italy); A. Carlotti, Institut de Planétologie et d'Astrophysique de Grenoble (France); M. Centrone, INAF - Osservatorio Astronomico di Roma (Italy); L. Close, J. Codona, Steward Observatory, The Univ. of Arizona (United States); S. Desidera, M. Dima, INAF - Osservatorio Astronomico di Padova (Italy); S. Esposito, INAF - Osservatorio Astronomico di Arcetri (Italy); D. Fantinel, G. Farisato, INAF - Osservatorio Astronomico di Padova (Italy); A. Fontana, INAF - Osservatorio Astronomico di Roma (Italy); W. Gaessler, Max-Planck-Institut für Astronomie (Germany); E. Giallongo, INAF - Osservatorio Astronomico di Roma (Italy); R. Gratton, INAF Osservatorio Astronomico di Padova (Italy); D. Greggio, INAF - Osservatorio Astronomico di Padova (Italy) and Univ. degli Studi di Padova (Italy); J. C. Guerra, O. Guyon, P. Hinz, Steward Observatory, The Univ. of Arizona (United States); F. Leone, Univ. degli Studi di Catania (Italy); F. Lisi, INAF - Osservatorio Astronomico di Arcetri (Italy); D. Magrin, INAF Osservatorio Astronomico di Padova (Italy); L. Marafatto, INAF - Osservatorio Astronomico di Padova (Italy) and Univ. di Padova (Italy); M. Munari, I. Pagano, INAF - Osservatorio Astrofisico di Catania (Italy); A. Puglisi, INAF - Osservatorio Astronomico di Arcetri (Italy); R. Ragazzoni, B. Salasnich, INAF - Osservatorio Astronomico di Padova (Italy); E. Sani, INAF Osservatorio Astronomico di Arcetri (Italy); S. Scuderi, INAF - Osservatorio Astrofisico di Catania (Italy); M. Stangalini, V. Testa, INAF - Osservatorio Astronomico di Roma (Italy); C. Verinaud, Institut de Planétologie et d'Astrophysique de Grenoble (France); V. Viotto, INAF - Osservatorio Astronomico di Padova (Italy)

9147 7K Gemini planet imager observational calibrations III: empirical measurement methods and applications of high-resolution microlens PSFs [9147-282]

P. Ingraham, Kavli Institute for Particle Astrophysics and Cosmology, Stanford Univ. (United States) and Univ. de Montréal (Canada); J.-B. Ruffio, SETI Institute (United States) and Institut Supérieur de l'Aéronautique et de l'Espace (Canada); M. D. Perrin, S. G. Wolff, Space Telescope Science Institute (United States); Z. H. Draper, Univ. of Victoria (Canada); J. Maire, Dunlap Institute for Astronomy and Astrophysics, Univ. of Toronto (Canada); F. Marchis, SETI Institute (United States); V. Fesquet, Gemini Observatory (Chile)

$91477 \mathrm{M}$ A laser locked Fabry-Perot etalon with $3 \mathrm{~cm} / \mathrm{s}$ stability for spectrograph calibration [9147-284]

Y. V. Gurevich, Yale Univ. (United States); J. Stürmer, Landessternwarte Heidelberg (Germany); C. Schwab, The Pennsylvania State Univ. (United States); T. Führer, Technische Univ. Darmstadt (Germany); S. K. Lamoreaux, Yale Univ. (United States); A. Quirrenbach, Landessternwarte Heidelberg (Germany); T. Walther, Technische Univ. Darmstadt (Germany)

914770 Gemini planet imager observational calibrations II: detector performance and calibration [9147-286]

P. Ingraham, Kavli Institute for Particle Astrophysics and Cosmology, Stanford Univ. (United States) and Univ. de Montréal (Canada); M. D. Perrin, Space Telescope Science Institute (United States); N. Sadakuni, Gemini Observatory (Chile); J.-B. Ruffio, SETI Institute (United States) and Institut Supérieur de l'Aéronautique et de l'Espace (France); J. Maire, Dunlap 
Institute for Astronomy and Astrophysics, Univ. of Toronto (Canada); J. Chilcote, J. Larkin, Univ. of California, Los Angeles (United States); F. Marchis, SETI Institute (United States);

R. Galicher, LESIA, CNRS, Observatoire de Paris, Univ. Paris Diderot (France); J. Weiss, Univ. of California, Los Angeles (United States)

9147 7P How to inject light efficiently into single-mode fibers [9147-287]

N. Jovanovic, Subaru Telescope, National Astronomical Observatory of Japan (United States); O. Guyon, Subaru Telescope, National Astronomical Observatory of Japan (United States) and Steward Observatory, Univ. of Arizona (United States); F. Martinache,

Observatoire de la Côte d'Azur (France); C. Schwab, The Pennsylvania State Univ. (United States); N. Cvetojevic, The Univ. of Sydney (Australia) and The Australian Astronomical Observatory (Australia)

9147 7Q Manufacturing, integration, and test results of the MATISSE cold optics bench [9147-288] F. C. M. Bettonvil, NOVA ASTRON (Netherlands) and Leiden Univ. (Netherlands); G. Kroes, T. Agoćs, NOVA ASTRON (Netherlands); A. van Duin, ASTRON (Netherlands); E. Elswijk, M. de Haan, R. ter Horst, J. Kragt, NOVA ASTRON (Netherlands); J. Kuindersma, ASTRON (Netherlands); R. Navarro, R. Roelfsema, M. Schuil, T. Tromp, L. Venema, NOVA ASTRON (Netherlands); F. van Kessel, Irmato Group (Netherlands); A. Jaskó, MTA Research Ctr. for Astronomy and Earth Sciences (Hungary)

9147 7R Concept and optical design of the cross-disperser module for CRIRES+ [9147-289] E. Oliva, A. Tozzi, D. Ferruzzi, INAF - Osservatorio Astrofisico di Arcetri (Italy); L. Origlia, INAF Osservatorio Astronomico di Bologna (Italy); A. Hatzes, R. Follert, T. Löwinger, Thüringer Landessternwarte Tautenburg (Germany); N. Piskunov, U. Heiter, M. Lockhart, T. Marquart, E. Stempels, Uppsala Univ. (Sweden); A. Reiners, G. Anglada-Escude, U. Seemann, Georg-August-Univ. Göttingen (Germany); R. J. Dorn, P. Bristow, D. Baade, B. Delabre, D. Gojak, J. Grunhut, B. Klein, M. Hilker, D. J. Ives, Y. Jung, H. U. Kaeufl, F. Kerber, J. L. Lizon, L. Pasquini, J. Paufique, E. Pozna, A. Smette, J. Smoker, E. Valenti, European Southern Observatory (Germany)

914775 Opto-mechanical design of a new cross dispersion unit for the CRIRES+ high resolution spectrograph for the VLT [9147-290]

J. L. Lizon, B. Klein, European Southern Observatory (Germany); E. Oliva, INAF - Osservatorio di Arcetri (Italy); T. Löwinger, Thüringer Landessternwarte Tautenburg (Germany); G. Anglada Escude, Georg-August-Univ. Göttingen (Germany); D. Baade, P. Bristow, R. J. Dorn, European Southern Observatory (Germany); R. Follert, Thüringer Landessternwarte Tautenburg (Germany); J. Grunhut, European Southern Observatory (Germany); A. Hatzes, Thüringer Landessternwarte Tautenburg (Germany); U. Heiter, Uppsala Univ. (Sweden); D. Ives, Y. Jung, F. Kerber, M. Lockhart, European Southern Observatory (Germany); T. Marquart, Uppsala Univ. (Sweden); L. Origlia, INAF - Osservatorio di Bologna (Italy); L. Pasquini, J. Paufique, European Southern Observatory (Germany); N. Piskunov, Uppsala Univ. (Sweden); E. Pozna, European Southern Observatory (Germany); A. Reiners, Georg-August-Univ. Göttingen (Germany); A. Smette, J. Smoker, European Southern Observatory (Germany); U. Seemann, Georg-August-Univ. Göttingen (Germany); E. Stempels, INAF - Osservatorio di Arcetri (Italy); E. Valenti, European Southern Observatory (Germany)

9147 7T HiJaK: the high-resolution J, $\mathrm{H}$ and K spectrometer [9147-291]

P. S. Muirhead, Z. J. Hall, M. J. Veyette, Boston Univ. (United States) 
$91477 \mathrm{U}$ Combining vector-phase coronagraphy with dual-beam polarimetry [9147-293]

F. Snik, G. Otten, M. Kenworthy, Leiden Observatory, Leiden Univ. (Netherlands); D. Mawet, European Southern Observatory (Chile); M. Escuti, North Carolina State Univ. (United States)

9147 7V An atmospheric turbulence and telescope simulator for the development of AOLI [9147-294]

M. Puga, R. López, Instituto de Astrofísica de Canarias (Spain) and Univ. de La Laguna (Spain); D. King, Univ. of Cambridge (United Kingdom); A. Oscoz, Instituto de Astrofísica de Canarias (Spain) and Univ. de La Laguna (Spain)

$91477 \mathrm{~W} \quad$ Stable Imaging for Astronomy (SIA) [9147-295]

M. Beaulieu, S. Ottogalli, O. Preis, Y. Bresson, J.-P. Rivet, L. Abe, F. Vakili, Univ. de Nice Sophia-Antipolis, Observatoire de la Côte d'Azur, CNRS (France)

$91477 X \quad$ Adapting a very high resolution echelle spectrograph to an 8 meter class telescope [9147-297]

R. O. Reynolds, L. Dettmann, Large Binocular Telescope Observatory (United States)

9147 7Y Development of compact and ultra-high-resolution spectrograph with multi-GHz optical frequency comb [9147-298]

M. Endo, The Univ. of Tokyo (Japan) and Exploratory Research for Advanced Technology (Japan); T. Sukegawa, Canon Inc. (Japan); A. Silva, Y. Kobayashi, The Univ. of Tokyo (Japan) and Exploratory Research for Advanced Technology (Japan)

914772 The habitable-zone planet finder calibration system [9147-299]

S. Halverson, S. Mahadevan, L. Ramsey, R. Terrien, A. Roy, C. Schwab, C. Bender, F. Hearty, E. Levi, The Pennsylvania State Univ. (United States); S. Osterman, Johns Hopkins Univ. Applied Physics Lab. (United States); G. Ycas, S. Diddams, National Institute of Standards and Technology (United States)

914780 A stable and inexpensive wavelength reference for precise wavelength calibration of radial velocity spectrographs [9147-300]

T. Feger, Macquarie Univ. (Australia); M. J. Ireland, The Australian National Univ. (Australia); J. Bento, C. Bacigalupo, Macquarie Univ. (Australia)

914781 Performance modeling of an upgraded NIRSPEC on Keck [9147-301]

E. C. Martin, M. P. Fitzgerald, I. S. McLean, Univ. of California, Los Angeles (United States);

S. M. Adkins, W. M. Keck Observatory (United States); T. Aliado, G. Brims, C. Johnson,

K. Magnone, E. Wang, J. Weiss, Univ. of California, Los Angeles (United States)

914782 Optical cavity characterization of the Tor Vergata Fabry-Pérot interferometer [9147-304]

L. Giovannelli, F. Berrilli, D. Del Moro, Univ. degli Studi di Roma "Tor Vergata" (Italy);

V. Greco, Istituto Nazionale di Ottica Applicata - CNR (Italy); R. Piazzesi, Univ. degli Studi di Roma "Tor Vergata" (Italy); A. Sordini, Istituto Nazionale di Ottica Applicata - CNR (Italy);

M. Stangalini, INAF - Osservatorio Astronomico di Roma (Italy)

914783 Gemini planet imager observational calibrations VII: on-sky polarimetric performance of the Gemini planet imager [9147-305]

S. J. Wiktorowicz, Univ. of California, Santa Cruz (United States); M. Millar-Blanchaer Univ. of Toronto (Canada); M. D. Perrin, Space Telescope Science Institute (United States); 
J. R. Graham, Univ. of California, Berkeley (United States); M. P. Fitzgerald, Univ. of California, Los Angeles (United States); J. Maire, Dunlap Institute for Astronomy and Astrophysics, Univ. of Toronto (Canada); P. Ingraham, Kavli Institute for Particle Astrophysics and Cosmology, Stanford Univ. (United States) and Univ. de Montréal (Canada); D. Savransky, Cornell Univ. (United States); B. A. Macintosh, Lawrence Livermore National Lab. (United States) and Stanford Univ. (United States); S. J. Thomas, NASA Ames Research Ctr. (United States); J. K. Chilcote, Univ. of California, Los Angeles (United States); Z. H. Draper, Univ. of Victoria (Canada); I. Song, Univ. of Georgia (United States); A. Cardwell, Gemini Observatory (Chile); S. J. Goodsell, Gemini Observatory (United States); M. Hartung, P. Hibon, F. Rantakyrö, N. Sadakuni, Gemini Observatory (Chile)

914784 Gemini planet imager observational calibrations V: astrometry and distortion [9147-306] Q. M. Konopacky, Dunlap Institute for Astronomy and Astrophysics, Univ. of Toronto (Canada); S. J. Thomas, NASA Ames Research Ctr. (United States); B. A. Macintosh, Stanford Univ. (United States) and Lawrence Livermore National Lab. (United States); D. Dillon, Univ. of California Observatories (United States); N. Sadakuni, Univ. of California Observatories (United States) and Gemini Observatory (Chile); J. Maire, Dunlap Institute for Astronomy and Astrophysics, Univ. of Toronto (Canada); M. Fitzgerald, Univ. of California, Los Angeles (United States); S. Hinkley, California Institute of Technology (United States); P. Kalas, Univ. of California, Berkeley (United States); T. Esposito, Univ. of California, Los Angeles (United States); C. Marois, NRC - Dominion Astrophysical Observatory (Canada); P. J. Ingraham, Stanford Univ. (United States); F. Marchis, SETI Institute (United States); M. D. Perrin, Space Telescope Science Institute (United States); J. R. Graham, J. J. Wang, Univ. of California, Berkeley (United States); R. J. De Rosa, Arizona State Univ. (United States) and Univ. of Exeter (United Kingdom); K. Morzinski, Steward Observatory, The Univ. of Arizona (United States); L. Pueyo, Space Telescope Science Institute (United States); J. K. Chilcote, J. E. Larkin, Univ. of California, Los Angeles (United States); D. Fabrycky, The Univ. of Chicago (United States); S. J. Goodsell, Gemini Observatory (Chile);

B. R. Oppenheimer, American Museum of Natural History (United States); J. Patience, Arizona State Univ. (United States); L. Saddlemyer, NRC - Dominion Astrophysical Observatory (Canada); A. Sivaramakrishnan, Space Telescope Science Institute (United States)

914785 Gemini planet imager observational calibrations VI: photometric and spectroscopic calibration for the integral field spectrograph [9147-307]

J. Maire, Dunlap Institute for Astronomy and Astrophysics, Univ. of Toronto (Canada); P. J. Ingraham, Kavli Institute for Particle Astrophysics and Cosmology, Stanford Univ. (United States); R. J. De Rosa, Arizona State Univ. (United States) and Univ. of Exeter (United Kingdom); M. D. Perrin, Space Telescope Science Institute (United States); A. Rajan, Arizona State Univ. (United States); D. Savransky, Cornell Univ. (United States); J. J. Wang, Univ. of California, Berkeley (United States); J.-B. Ruffio, SETI Institute (United States); S. G. Wolff, Johns Hopkins Univ. (Canada); J. K. Chilcote, Univ. of California, Los Angeles (United States); R. Doyon, Univ. de Montréal (Canada); J. R. Graham, Univ. of California, Berkeley (United States); A. Z. Greenbaum, Johns Hopkins Univ. (United States); Q. M. Konopacky, Dunlap Institute for Astronomy and Astrophysics, Univ. of Toronto (Canada); J. E. Larkin, Univ. of California, Los Angeles (United States); B. A. Macintosh, Kavli Institute for Particle Astrophysics and Cosmology, Stanford Univ. (United States) and Lawrence Livermore National Lab. (United States); C. Marois, NRC - Herzberg Institute of Astrophysics (Canada); M. Millar-Blanchaer, Univ. of Toronto (Canada); J. Patience, Arizona State Univ. (United States); L. A. Pueyo, A. Sivaramakrishnan, Space Telescope Science Institute (United States); S. J. Thomas, NASA Ames Research Ctr. (United States); J. L. Weiss, Univ. of California, Los Angeles (United States) 
914786 A robotic, compact, and extremely high resolution optical spectrograph for a close-in super-Earth survey [9147-308]

J. Ge, S. Powell, B. Zhao, F. Varosi, B. Ma, S. Sithajan, J. Liu, R. Li, N. Grieves, S. Schofield, L. Avner, H. Jakeman, W. A. Yoder, J. A. Gittelmacher, M. A. Singer, Univ. of Florida (United

States); M. Muterspaugh, M. Williamson, J. E. Maxwell, Tennessee State Univ. (United States)

914787 Characterizing instrumental effects on polarization at a Nasmyth focus using NaCo [9147-309]

J. de Boer, European Southern Observatory (Chile) and Leiden Observatory, Leiden Univ. (Netherlands); J. H. Girard, D. Mawet, European Southern Observatory (Chile); F. Snik, C. U. Keller, Leiden Observatory, Leiden Univ. (Netherlands); J. Milli, European Southern Observatory (Chile) and IPAG, Univ. Joseph Fourier, CNRS (France)

\section{Part Five}

914788 The upgrade of a high dispersion spectro-polarimeter, VESPoIA: new circular polarimetry mode and extremely high resolution mode [9147-310]

T. Arasaki, Kyoto Sangyo Univ. (Japan); Y. Ikeda, Photocoding (Japan); N. Akika,

H. Kawakita, Kyoto Sangyo Univ. (Japan)

914789 FIDEOS: a high resolution echelle spectrograph for the ESO $1 \mathrm{~m}$ telescope at La Silla [9147-311]

M. Tala, A. Berdja, M. Jones, L. Vanzi, S. Ropert, Pontificia Univ. Católica de Chile (Chile); M. Flores, Univ. de Santiago de Chile (Mexico); C. Viscasillas, Univ. Católica de Avila (Spain)

$91478 C \quad H A R P S-N @$ TNG, two year harvesting data: performances and results [9147-314] R. Cosentino, INAF - Telescopio Nazionale Galileo (Spain); C. Lovis, F. Pepe, Observatoire Astronomique de l'Univ. de Genève (Switzerland); A. C. Cameron, Univ. of St. Andrews (United Kingdom); D. W. Latham, Harvard-Smithsonian Ctr. for Astrophysics (United States); E. Molinari, INAF - Telescopio Nazionale Galileo (Spain); S. Udry, Observatoire Astronomique de I'Univ. de Genève (Switzerland); N. Bezawada, UK Astronomy Technology Ctr. (United Kingdom); N. Buchschacher, Observatoire Astronomique de l'Univ. de Genève (Switzerland); P. Figueira, Natural History Museum of Denmark (Denmark); M. Fleury, Observatoire Astronomique de l'Univ. de Genève (Switzerland); A. Ghedina, INAF Telescopio Nazionale Galileo (Spain); A. G. Glenday, Harvard-Smithsonian Ctr. for Astrophysics (United States); M. Gonzalez, J. Guerra, INAF - Telescopio Nazionale Galileo (Spain); D. Henry, UK Astronomy Technology Ctr. (United Kingdom); I. Hughes, C. Maire, F. Motalebi, Observatoire Astronomique de l'Univ. de Genève (Switzerland); D. Phillips, Harvard-Smithsonian Ctr. for Astrophysics (United States)

9147 8D Development of infrared echelle spectrograph and mid-infrared heterodyne spectrometer on a small telescope at Haleakala, Hawaii for planetary observation [9147-315]

T. Sakanoi, Y. Kasaba, M. Kagitani, H. Nakagawa, Tohoku Univ. (Japan); J. Kuhn, S. Okano, Institute for Astronomy, Univ. of Hawaili (United States)

9147 8E ERIS: the exoplanet high-resolution image simulator for CHARIS [9147-316]

M. A. Limbach, T. D. Groff, N. J. Kasdin, Princeton Univ. (United States); T. Brandt, Institute for Advanced Studies (United States); K. Mede, The Univ. of Tokyo (Japan); C. Loomis,

Princeton Univ. (United States); M. Hayashi, N. Takato, National Astronomical Observatory of Japan (Japan) 
$91478 \mathrm{~F}$ The solar system at 10 parsec: exploiting the ExAO of LBT in the visual wavelengths [9147-317]

M. Stangalini, F. Pedichini, M. Centrone, INAF - Osservatorio Astronomico di Roma (Italy); S. Esposito, INAF - Osservatorio Astrofisico di Arcetri (Italy); J. Farinato, INAF - Osservatorio Astronomico di Padova (Italy); E. Giallongo, INAF - Osservatorio Astronomico di Roma (Italy); F. Quirós-Pacheco, E. Pinna, INAF - Osservatorio Astrofisico di Arcetri (Italy)

$91478 \mathrm{H} \quad$ SPRAT: Spectrograph for the Rapid Acquisition of Transients [9147-319]

A. S. Piascik, I. A. Steele, S. D. Bates, C. J. Mottram, R. J. Smith, R. M. Barnsley, B. Bolton, Liverpool John Moores Univ. (United Kingdom)

$914781 \quad$ DIPOL-2: a double image high precision polarimeter [9147-321]

V. Piirola, A. Berdyugin, Univ. of Turku (Finland); S. Berdyugina, Kiepenheuer-Institut für Sonnenphysik (Germany)

$91478 \mathrm{~J}$ H.O.R.S. a new visiting instrument for G.T.C. based on the Utrecht Echelle Spectrograph [9147-322]

J. Peñate, F. Gracia, C. Allende, J. Calvo, S. Santana, Instituto de Astrofísica de Canarias (Spain)

9147 8K ESPRESSO front end exposure meter: a chromatic approach to radial velocity correction [9147-323]

M. Landoni, M. Riva, INAF - Osservatorio Astronomico di Brera (Italy); F. Pepe, Observatoire de Genève (Switzerland); P. Conconi, F. M. Zerbi, INAF - Osservatorio Astronomico di Brera (Italy); A. Cabral, Univ. de Lisboa (Portugal); S. Cristiani, INAF - Osservatorio Astronomico di Trieste (Italy); D. Megevand, Observatoire de Genève (Switzerland)

9147 8L First calibration and visible wavelength observations of Khayyam: a tunable spatial heterodyne spectroscopy (SHS) [9147-324]

S. Hosseini, Univ. of California, Davis (United States); W. Harris, The Univ. of Arizona (United States)

$91478 \mathrm{M}$ The metrology system for the multi-object optical and near-infrared spectrograph MOONS [9147-325]

M. Torres-Torriti, L. Vanzi, R. Dünner, F. Belmar, L. Dauvin, T.-C. Chen, Pontificia Univ. Católica de Chile (Chile); M. Cirasuolo, W. Taylor, H. Schnetler, D. Montgomery, P. Rees, UK Astronomy Technology Ctr. (United Kingdom); A. Cabral, Univ. de Lisboa (Portugal)

9147 8N Green astro-comb for HARPS-N [9147-326]

N. Langellier, Harvard Univ. (United States); C.-H. Li, A. G. Glenday, Harvard-Smithsonian Ctr. for Astrophysics (United States); G. Chang, Massachusetts Institute of Technology (United States) and Hamburg Univ. (Germany); H.-W. Chen, J. Lim, Massachusetts Institute of Technology (United States); G. Furesz, Harvard-Smithsonian Ctr. for Astrophysics (United States); F. Kärtner, Massachusetts Institute of Technology (United States) and Hamburg Univ. (Germany); D. F. Phillips, Harvard-Smithsonian Ctr. for Astrophysics (United States); D. Sasselov, Harvard Univ. (United States) and Harvard-Smithsonian Ctr. for Astrophysics (United States); A. Szentgyorgyi, Harvard-Smithsonian Ctr. for Astrophysics (United States); R. Walsworth, Harvard Univ. (United States) and Harvard-Smithsonian Ctr. for Astrophysics (United States) 
914780 A coronagraph based on two spatial light modulators for active amplitude apodizing and phase corrections [9147-328]

J. Dou, Nanjing Institute of Astronomical Optics and Technology (China); D. Ren, Nanjing Institute of Astronomical Optics and Technology (China) and California State Univ., Northridge (United States); X. Zhang, Y. Zhu, G. Zhao, Z. Wu, R. Chen, Nanjing Institute of Astronomical Optics and Technology (China); C. Liu, F. Yang, C. Yang, Nanjing Institute of Astronomical Optics and Technology (China) and Univ. of Chinese Academy of Sciences (China)

$91478 \mathrm{P} \quad$ Novel infrared polarimeter for the ESO CRIRES+ instrument [9147-329]

M. Lockhart, N. Piskunov, E. Stempels, Uppsala Univ. (Sweden); M. Escuti, North Carolina State Univ. (United States); E. Oliva, INAF - Osservatorio Astrofisico di Arcetri (Italy); H.-U. Käufl, European Southern Observatory (Germany); U. Heiter, T. Marquart, Uppsala Univ. (Sweden); G. Anglada-Escude, Georg-August-Univ. Göttingen (Germany); D. Baade, P. Bristow, R. J. Dorn, European Southern Observatory (Germany); R. Follert, Thüringer Landessternwarte Tautenburg (Germany); D. Gojak, J. H. Grunhut, European Southern Observatory (Germany); A. Hatzes, Thüringer Landessternwarte Tautenburg (Germany); M. Hilker, D. Ives, Y. Jung, F. Kerber, B. Klein, J.-L. Lizon, European Southern Observatory (Germany); T. Löwinger, Thüringer Landessternwarte Tautenburg (Germany); L. Origlia, INAF - Osservatorio Astrofisico di Arcetri (Italy); L. Pasquini, J. Paufique, E. Pozna, European Southern Observatory (Germany); A. Reiners, U. Seemann, Georg-August-Univ. (Germany); A. Smette, J. Smoker, E. Valenti, European Southern Observatory (Germany)

9147 8Q ESPRESSO Coudé-Train: complexities of a simultaneous optical feeding from the four VLT unit telescopes [9147-330]

A. Cabral, M. Abreu, J. Coelho, R. Gomes, Ctr. de Astronomia e Astrofísica da Univ. de Lisboa (Portugal); M. Monteiro, Ctr. de Astrofísica da Univ. do Porto (Portugal); A. Oliveira, P. Santos, Univ. de Lisboa (Portugal); G. Ávila, B.-A. Delabre, European Southern Observatory (Germany); M. Riva, INAF - Osservatorio Astronomico di Brera (Italy); P. Di Marcantonio, I. Coretti, INAF - Osservatorio Astronomico di Trieste (Italy); N. C. Santos, Univ. de Lisboa (Portugal) and Univ. do Porto (Portugal); F. Zerbi, INAF - Osservatorio Astronomico di Brera (Italy); D. Mégevand, Observatoire de I'Univ. de Genève (Switzerland)

$91478 R \quad$ Fast multichannel astronomical photometer based on silicon photo multipliers mounted at the Telescopio Nazionale Galileo [9147-371]

F. Ambrosino, F. Meddi, C. Rossi, S. Sclavi, Univ. degli Studi di Roma La Sapienza (Italy);

R. Nesci, INAF - Istituto di Astrofisica e Planetologia Spaziali (Italy); I. Bruni, INAF Osservatorio Astronomico di Bologna (Italy); A. Ghedina, L. Riverol, L. Di Fabrizio, INAF Fundación Galileo Galilei (Spain)

$91478 S \quad$ Photometric study of the Paranal observatory using MASS database [9147-373] A. Abahamid, Univ. Cadi Ayyad (Morocco); M. Sarazin, European Southern Observatory (Germany); G. Lombardi, European Southern Observatory (Chile)

$91478 \mathrm{~T} \quad$ First performance of the GeMS+GMOS system [9147-65]

P. Hibon, Gemini South Observatory (Chile); B. Neichel, Lab. d'Astrophysique de Marseille (France); V. Garrel, Gemini South Observatory (Chile); B. Prout, F. Rigaut, The Australian National Univ. (Australia); A. Koning, Univ. of Victoria (Canada); G. Sivo, G. Gimeno, R. Carrasco, C. Winge, P. Pessev, A. Serio, G. Arriagada, Gemini South Observatory (Chile) 
$91478 U$ Design and analysis of a large-diameter precision optical mount for NFIRAOS [9147-303]

J. Fitzsimmons, A. Hill, National Research Council Canada (Canada)

9147 8V HARMONI instrument control electronics [9147-331]

J. V. Gigante, L. F. Rodríguez, Instituto de Astrofísica de Canarias (Spain); G. Zins, Institut de Planétologie et d'Astrophysique de Grenoble (France); H. Schnetler, UK Astronomy Technology Ctr. (United Kingdom); A. Pecontal, Ctr. de Recherche Astrophysique de Lyon (France); J. M. Herreros, Instituto de Astrofísica de Canarias (Spain); F. Clarke, Univ. of Oxford (United Kingdom); I. Bryson, UK Astronomy Technology Ctr. (United Kingdom); N. Thatte, Univ. of Oxford (United Kingdom)

$91478 \mathrm{~W}$ A novel systems engineering approach to the design of a precision radial velocity spectrograph: the GMT-Consortium Large Earth Finder (G-CLEF) [9147-333]

W. Podgorski, Harvard-Smithsonian Ctr. for Astrophysics (United States); J. Bean, The Univ. of Chicago (United States); H. Bergner, Harvard-Smithsonian Ctr. for Astrophysics (United States); M.-Y. Chun, Korea Astronomy and Space Science Institute (Korea, Republic of); J. Crane, Carnegie Observatories (United States); I. Evans, J. Evans, G. Furesz, HarvardSmithsonian Ctr. for Astrophysics (United States); D. Guzman, Pontificia Univ. Católica de Chile (Chile); K.-M. Kim, Korea Astronomy and Space Science Institute (Korea, Republic of); K. McCracken, M. Mueller, T. Norton, Harvard-Smithsonian Ctr. for Astrophysics (United States); C. Park, Korea Astronomy and Space Science Institute (Korea, Republic of); S. Park, D. Plummer, A. Szentgyorgyi, Harvard-Smithsonian Ctr. for Astrophysics (United States); A. Uomoto, Carnegie Observatories (United States); I.-S. Yuk, Korea Astronomy and Space Science Institute (Korea, Republic of)

$91478 \mathrm{X}$ The infrared imaging spectrograph (IRIS) for TMT: volume phase holographic grating performance testing and discussion [9147-334]

S. Chen, Dunlap Institute for Astronomy and Astrophysics, Univ. of Toronto (Canada); E. Meyer, S. A. Wright, Dunlap Institute for Astronomy and Astrophysics, Univ. of Toronto (Canada) and Univ. of Toronto (Canada); A. M. Moore, Caltech Optical Observatories (United States); J. E. Larkin, Univ. of California, Los Angeles (United States); J. Maire, Dunlap Institute for Astronomy and Astrophysics, Univ. of Toronto (Canada); E. Mieda, Dunlap Institute for Astronomy and Astrophysics, Univ. of Toronto (Canada) and Univ. of Toronto (Canada); L. Simard, NRC - Dominion Astrophysical Observatory (Canada)

$91478 Y$ Development of a subwavelength grating vortex coronagraph of topological charge 4 (SGVC4) [9147-335]

C. Delacroix, O. Absil, B. Carlomagno, P. Piron, Univ. de Liège (Belgium); P. Forsberg, M. Karlsson, Uppsala Univ. (Sweden); D. Mawet, European Southern Observatory (Chile); S. Habraken, J. Surdej, Univ. de Liège (Belgium)

$91478 Z$ Integral field spectroscopy of high redshift galaxies with the HARMONI spectrograph on the European Extremely Large Telescope [9147-336]

S. Kendrew, S. Zieleniewski, N. Thatte, J. Devriendt, R. Houghton, Univ. of Oxford (United Kingdom); T. Fusco, ONERA (France); M. Tecza, F. Clarke, K. O'Brien, Univ. of Oxford (United Kingdom) 
914790 Trade-off study for high resolution spectroscopy in the near infrared with ELT telescopes: seeing-limited vs. diffraction limited instruments [9147-337]

N. Sanna, E. Oliva, F. Massi, G. Cresci, INAF - Osservatorio Astrofisico di Arcetri (Italy);

L. Origlia, INAF - Osservatorio Astronomico di Bologna (Italy)

$914791 \quad$ Simulations of high-z galaxy observations with an ELT-MOS [9147-338]

K. Disseau, M. Puech, H. Flores, F. Hammer, Y. Yang, GEPI, Observatoire de Paris, CNRS, Univ. Paris Diderot (France); L. Pentericci, INAF - Osservatorio Astronomico di Roma (Italy)

914792 All-sky homogeneity of precipitable water vapour over Paranal [9147-339]

R. R. Querel, National Institute of Water and Atmospheric Research (New Zealand) and Univ. de Chile (Chile); F. Kerber, European Southern Observatory (Germany)

914793 Simulating observations with HARMONI: the integral field spectrograph for the European Extremely Large Telescope [9147-340]

S. Zieleniewski, N. Thatte, S. Kendrew, R. Houghton, M. Tecza, F. Clarke, Univ. of Oxford

(United Kingdom); T. Fusco, ONERA (France); M. Swinbank, Durham Univ. (United Kingdom)

914794 The MANIFEST fibre positioning system for the Giant Magellan Telescope [9147-341] J. S. Lawrence, D. M. Brown, J. Brzeski, S. Case, Australian Astronomical Observatory (Australia); M. Colless, The Australian National Univ. (Australia); T. Farrell, L. Gers, J. Gilbert, M. Goodwin, Australian Astronomical Observatory (Australia); G. Jacoby, Giant Magellan Telescope (United States) and Carnegie Observatories (United States); A. M. Hopkins, M. Ireland, K. Kuehn, N. P. F. Lorente, S. Miziarski, R. Muller, V. Nichani, A. Rakman, Australian Astronomical Observatory (Australia); S. Richards, The Univ. of Sydney (Australia); W. Saunders, N. F. Staszak, J. Tims, M. Vuong, L. Waller, Australian Astronomical Observatory (Australia)

914795 A mechanical design concept for EAGLE on the revised E-ELT [9147-342] C. M. Dubbeldam, Durham Univ. (United Kingdom)

914796 Science case and requirements for the MOSAIC concept for a multi-object spectrograph for the European Extremely Large Telescope [9147-343]

C. J. Evans, UK Astronomy Technology Ctr. (United Kingdom); M. Puech, GEPI, Observatoire de Paris, CNRS, Univ. Paris Diderot (France); B. Barbuy, Univ. de São Paulo (Brazil); P. Bonifacio, GEPI, Observatoire de Paris, CNRS, Univ. Paris Diderot (France); J.-G. Cuby, Aix Marseille Univ., CNRS, Lab. d'Astrophysique de Marseille (France); E. Guenther, Thüringer Landessternwarte Tautenburg (Germany); F. Hammer, P. Jagourel, GEPI, Observatoire de Paris, CNRS, Univ. Paris Diderot (France); L. Kaper, Univ. van Amsterdam (Netherlands); S. L. Morris, Durham Univ. (United Kingdom); J. Afonso, Observatório Astronómico de Lisboa (Portugal); P. Amram, Aix Marseille Univ., CNRS, Lab. d'Astrophysique de Marseille (France); H. Aussel, CEA, AIM, Service d'Astrophysique (France); A. Basden, Durham Univ. (United Kingdom); N. Bastian, Liverpool John Moores Univ. (United Kingdom); G. Battaglia, Instituto de Astrofísica de Canarias (Spain); B. Biller, The Royal Observatory, Edinburgh (United Kingdom); N. Bouché, Univ. de Toulouse, UPS-OMP, CNRS (France); E. Caffau, GEPI, Observatoire de Paris, CNRS, Univ. Paris Diderot (France); S. Charlot, UPMC-CNRS, Institut d'Astrophysique de Paris (France); Y. Clenet, LESIA, Observatoire de Paris, CNRS-UPMC, Univ. Paris-Diderot (France); F. Combes, LERMA, Observatoire de Paris, CNRS (France); C. Conselice, The Univ. of Nottingham (United Kingdom); T. Contini, Univ. de Toulouse, UPSOMP, CNRS (France); G. Dalton, Univ. of Oxford (United Kingdom) and Rutherford Appleton Lab. (United Kingdom); B. Davies, Liverpool John Moores Univ. (United States); K. Disseau, GEPI, Observatoire de Paris, CNRS, Univ. Paris Diderot (France); J. Dunlop, The 
Royal Observatory, Edinburgh (United Kingdom); F. Fiore, INAF - Osservatorio Astronomico di Roma (Italy); H. Flores, GEPI, Observatoire de Paris, CNRS, Univ. Paris Diderot (France); T. Fusco, ONERA (France); D. Gadotti, European Southern Observatory (Chile); A. Gallazzi, INAF - Osservatorio Astrofisico di Arcetri (Italy); E. Giallongo, INAF - Osservatorio

Astronomico di Roma (Italy); T. Gonçalves, Observatório do Valongo (Brazil); D. Gratadour, LESIA, Observatoire de Paris, CNRS-UPMC, Univ. Paris-Diderot (France); V. Hill, Observatoire de la Côte d'Azur (France); M. Huertas-Company, GEPI, Observatoire de Paris, CNRS, Univ. Paris Diderot (France); R. Ibata, Observatoire de Strasbourg (France); S. Larsen, Radboud Univ. Nijmegen (Netherlands); O. Le Fèvre, Aix Marseille Univ., CNRS, Lab. d'Astrophysique de Marseille (France); B. Lemasle, Univ. von Amsterdam (Netherlands); C. Maraston, Univ. of Portsmouth (United Kingdom); S. Mei, GEPI, Observatoire de Paris, CNRS, Univ. Paris Diderot (France); Y. Mellier, UPMC-CNRS, Institut d'Astrophysique de Paris (France); G. Östlin, Stockholm Univ. (Sweden); T. Paumard, LESIA, Observatoire de Paris, CNRS-UPMC, Univ. Paris-Diderot (France); R. Pello, Univ. de Toulouse, UPS-OMP, CNRS (France); L. Pentericci, INAF - Osservatorio Astronomico di Roma (Italy); P. Petitjean, UPMC-CNRS, Institut d'Astrophysique de Paris (France); M. Roth, Leibniz-Institut für Astrophysik Potsdam (Germany); D. Rouan, LESIA, Observatoire de Paris, CNRS-UPMC, Univ. Paris-Diderot (France); D. Schaerer, Observatoire de Genève, Univ. de Genève (Switzerland); E. Telles, Observatorio Nacional (Brazil); S. Trager, Univ. of Groningen (Netherlands); N. Welikala, Univ. of Oxford (United Kingdom); S. Zibetti, INAF - Osservatorio Astrofisico di Arcetri (Italy); B. Ziegler, Univ. Wien (Austria)

914797 The spectrograph units for the HARMONI integral field spectrograph [9147-344]

K. O'Brien, J. R. Allen, J. D. Lynn, N. A. Thatte, Univ. of Oxford (United Kingdom); I. Bryson, UK Astronomy Technology Ctr. (United Kingdom); F. Clarke, Univ. of Oxford (United Kingdom); H. Schnetler, UK Astronomy Technology Ctr. (United Kingdom); M. Tecza, Univ. of Oxford (United Kingdom)

914799 Mid-IR AGPMs for ELT applications [9147-346]

B. Carlomagno, C. Delacroix, O. Absil, Univ. de Liège (Belgium); P. Forsberg, Uppsala Univ. (Sweden); S. Habraken, A. Jolivet, Univ. de Liège (Belgium); M. Karlsson, Uppsala Univ. (Sweden); D. Mawet, European Southern Observatory (Chile); P. Piron, J. Surdej, Univ. de Liège (Belgium); E. Vargas Catalan, Uppsala Univ. (Sweden)

91479A The opto-mechanical design of the GMT-consortium large earth finder (G-CLEF) [9147-347] M. Mueller, D. Baldwin, Harvard-Smithsonian Ctr. for Astrophysics (United States); J. Bean, The Univ. of Chicago (United States); H. Bergner, Harvard-Smithsonian Ctr. for Astrophysics (United States); B. Bigelow, GMTO Corp. (United States); M.-Y. Chun, Korea Astronomy and Space Science Institute (Korea, Republic of); J. Crane, Carnegie Observatories (United States); J. Foster, G. Fürész, T. Gauron, Harvard-Smithsonian Ctr. for Astrophysics (United States); D. Guzman, Pontificia Univ. Católica de Chile (Chile); E. Hertz, Harvard-Smithsonian Ctr. for Astrophysics (United States); A. Jordán, Pontificia Univ. Católica de Chile (Chile); K.-M. Kim, Korea Astronomy and Space Science Institute (Korea, Republic of); K. McCracken, T. Norton, M. Ordway, Harvard-Smithsonian Ctr. for Astrophysics (United States); C. Park, Korea Astronomy and Space Science Institute (Korea, Republic of); S. Park, W. Podgorski, A. Szentgyorgyi, Harvard-Smithsonian Ctr. for Astrophysics (United States); A. Uomoto, Carnegie Observatories (United States); I.-S. Yuk, Korea Astronomy and Space Science Institute (Korea, Republic of) 
91479B SKA pulsar search: technological challenges and best algorithms development [9147-348] C. Baffa, INAF - Osservatorio Astrofisico di Arcetri (Italy)

$91479 C$ The infrared imaging spectrograph (IRIS) for TMT: reflective ruled diffraction grating performance testing and discussion [9147-349]

E. Meyer, Dunlap Institute for Astronomy and Astrophysics, Univ. of Toronto (Canada) and Univ. of Toronto (Canada); S. Chen, Dunlap Institute for Astronomy and Astrophysics, Univ. of Toronto (Canada); S. A. Wright, Dunlap Institute for Astronomy and Astrophysics, Univ. of Toronto (Canada) and Univ. of Toronto (Cambodia); A. M. Moore, Caltech Optical Observatories (United States); J. E. Larkin, Univ. of California, Los Angeles (United States); L. Simard, NRC - Dominion Astrophysical Observatory (Canada); J. Maire, Dunlap Institute for Astronomy and Astrophysics, Univ. of Toronto (Canada); E. Mieda, Dunlap Institute for Astronomy and Astrophysics, Univ. of Toronto (Canada) and Univ. of Toronto (Canada); J. Gordon, Univ. of Toronto (Canada)

91479D Three possible types of coronagraphs for the E-ELT PCS instrument [9147-350] A. Carlotti, IPAG, CNRS (France); M. N'Diaye, L. Pueyo, Space Telescope Science Institute (United States); D. Mawet, European Southern Observatory (Chile)

$91479 \mathrm{E} \quad$ The high contrast imaging modes of MICADO [9147-351]

P. Baudoz, A. Boccaletti, S. Lacour, R. Galicher, Y. Clénet, D. Gratadour, É. Gendron, T. Buey, G. Rousset, Lab. d'Etudes Spatiales et d'Instrumentation en Astrophysique, Observatoire de Paris-Meudon (France); M. Hartl, R. Davies, Max-Planck-Institut für extraterrestrische Physik (Germany)

$91479 F \quad$ An aperture masking mode for the MICADO instrument [9147-352]

S. Lacour, P. Baudoz, E. Gendron, A. Boccaletti, R. Galicher, Y. Clénet, D. Gratadour, T. Buey, G. Rousset, LESIA, Observatoire de Paris (France); M. Hartl, R. Davies, Max-PlanckInstitut für extraterrestrische Physik (Germany)

914796 The G-CLEF spectrograph optical design [9147-353]

G. Fürész, Harvard-Smithsonian Ctr. for Astrophysics (United States); H. Epps, Univ. of California Observatories, Lick Observatory (United States) and Univ. of California Santa Cruz (United States); S. Barnes, Stuart Barnes Optical Design (Netherlands); W. Podgorski, A. Szentgyorgyi, M. Mueller, D. Baldwin, Harvard-Smithsonian Ctr. for Astrophysics (United States); J. Bean, The Univ. of Chicago (United States); H. Bergner, Harvard-Smithsonian Ctr. for Astrophysics (United States); M.-Y. Chun, Korea Astronomy and Space Science Institute (Korea, Republic of); J. Crane, Carnegie Observatories (United States); J. Evans, I. Evans, J. Foster, T. Gauron, Harvard-Smithsonian Ctr. for Astrophysics (United States); D. Guzman, Pontificia Univ. Católica de Chile (Chile); E. Hertz, Harvard-Smithsonian Ctr. for Astrophysics (United States); A. Jordán, Pontificia Univ. Catolica de Chile (Chile); K.-M. Kim, Korea Astronomy and Space Science Institute (Korea, Republic of); K. McCracken, T. Norton, M. Ordway, Harvard-Smithsonian Ctr. for Astrophysics (United States); C. Park, Korea Astronomy and Space Science Institute (Korea, Republic of); S. Park, D. Plummer, HarvardSmithsonian Ctr. for Astrophysics (United States); A. Uomoto, Carnegie Observatories (United States); I.-S. Yuk, Korea Astronomy and Space Science Institute (Korea, Republic of)

$91479 \mathrm{H}$ On-instrument wavefront sensor design for the TMT infrared imaging spectrograph (IRIS) update [9147-354]

J. Dunn, V. Reshetov, J. Atwood, J. Pazder, B. Wooff, D. Loop, L. Saddlemyer, NRC Herzberg Institute of Astrophysics (Canada); A. M. Moore, Caltech Optical Observatories (United States); J. E. Larkin, Univ. of California, Los Angeles (United States) 
$914791 \quad$ DKIST visible broadband imager interference filters [9147-355]

F. Wöger, National Solar Observatory (United States)

$91479 \mathrm{~J}$ Optical and mechanical design of the fore-optics of HARMONI [9147-356]

J. Sánchez-Capuchino, E. Hernández, A. Bueno, J. M. Herreros, Instituto de Astrofísica de Canarias (Spain); N. Thatte, Univ. of Oxford (United Kingdom); I. Bryson, UK Astronomy Technology Ctr. (United Kingdom); F. Clarke, M. Tecza, Univ. of Oxford (United Kingdom)

9147 9K Beating the heat! Automated characterization of piezoelectric tubes for Starbugs [9147-357]

R. Piersiak, Stony Brook Univ. (United States); M. Goodwin, J. Gilbert, R. Muller, Australian Astronomical Observatory (Australia)

9147 9L Wireless software update system based on Zigbee for LAMOST [9147-358]

S. Li, Y. GU, Y. Jin, C. Zhai, Univ. of Science and Technology of China (China)

$91479 \mathrm{M}$ Cryogenic testing of components for the HARMONI spectrograph [9147-359]

J. R. Allen, K. O'Brien, J. D. Lynn, N. A. Thatte, Univ. of Oxford (United Kingdom); I. Bryson, UK Astronomy Technology Ctr. (United Kingdom); F. Clarke, Univ. of Oxford (United Kingdom); H. Schnetler, UK Astronomy Technology Ctr. (United Kingdom); M. Tecza, Univ. of Oxford (United Kingdom)

$91479 \mathrm{~N}$ The fiber-fed preslit of GIANO at T.N.G. [9147-360]

A. Tozzi, E. Oliva, INAF - Osservatorio Astrofisico di Arcetri (Italy); L. Origlia, INAF Osservatorio Astronomico di Bologna (Italy); C. Baffa, V. Biliotti, G. Falcini, E. Giani, M. Iuzzolino, F. Massi, N. Sanna, INAF - Osservatorio Astrofisico di Arcetri (Italy); S. Scuderi, INAF - Osservatorio Astrofisico di Catania (Italy); M. Sozzi, INAF - Osservatorio Astrofisico di Arcetri (Italy)

914790 Simultaneous phase and amplitude retrieval with COFFEE: from theory to laboratory results [9147-363]

B. Paul, ONERA (France), Lab. d'Astrophysique de Marseille, Univ. Aix-Marseille, CNRS (France), and PHASE, Observatoire de Paris, CNRS, Univ. Diderot (France); J.-F. Sauvage, L. M. Mugnier, ONERA (France) and PHASE, Observatoire de Paris, CNRS, Univ. Diderot (France); K. Dohlen, Lab. d'Astrophysique de Marseille, Univ. Aix-Marseille, CNRS (France) and PHASE, Observatoire de Paris, CNRS, Univ. Diderot (France); T. Fusco, ONERA (France), Lab. d'Astrophysique de Marseille, Univ. Aix-Marseille, CNRS (France), and PHASE, Observatoire de Paris, CNRS, Univ. Diderot (France); M. Ferrari, Lab. d'Astrophysique de Marseille, Univ. Aix-Marseille, CNRS (France) and PHASE, Observatoire de Paris, CNRS, Univ. Diderot (France)

9147 9P Infrared differential imager and spectrograph for SPHERE: performance assessment for onsky operation [9147-365]

M. Langlois, Ctr. de Recherche Astronomique de Lyon, Observatoire de Lyon, CNRS, Univ. de Lyon 1 (France) and Ecole Normale Supérieure de Lyon (France); A. Vigan, K. Dohlen, C. Moutou, Aix-Marseille Univ., CNRS, Lab. d'Astrophysique de Marseille (France);

J.-L. Beuzit, Institut de Planétologie et d'Astrophysique de Grenoble (France); A. Boccaletti, LESIA, Observatoire de Paris (France); M. Carle, A. Costille, Aix-Marseille Univ., CNRS, Lab. d'Astrophysique de Marseille (France); R. Dorn, European Southern Observatory (Germany); L. Gluck, Institut de Planétologie et d'Astrophysique de Grenoble (France); 
C. Gry, Aix-Marseille Univ., CNRS, Lab. d'Astrophysique de Marseille (France); N. Hubin, European Southern Observatory (Germany); M. Feldt, Max-Planck-Institut für Astronomie (Germany); M. Kasper, European Southern Observatory (Germany); F. Madec,

D. Le Mignant, Aix-Marseille Univ., CNRS, Lab. d'Astrophysique de Marseille (France); J.-L. Lizon, European Southern Observatory (Germany); D. Mouillet, Institut de Planétologie et d'Astrophysique de Grenoble (France); A. Origné, Aix-Marseille Univ., CNRS, Lab. d'Astrophysique de Marseille (France); P. Puget, Institut de Planétologie et d'Astrophysique de Grenoble (France); J.-F. Sauvage, ONERA (France); F. Wildi, Observatoire Astronomique de I'Univ. de Genève (Switzerland); A. Zurlo, Aix-Marseille Univ., CNRS, Lab. d'Astrophysique de Marseille (France)

91479Q Fully cryogenic phased array prototype camera for the Arecibo radio telescope [9147-366] G. Cortes-Medellin, A. Vishwas, S. C. Parshley, D. B. Campbell, Cornell Univ. (United States)

9147 9R LOTUCE2: a dome-seeing instrument for the E-ELT [9147-367]

A. Berdja, D. Guzman, N. Saez, N. David, N. Dubost, Pontificia Univ. Católica de Chile (Chile); M. Sarazin, European Southern Observatory (Germany); A. Ziad, Lab. J.L. Lagrange, Univ. Nice Sophia-Antipolis, CNRS, OCA (France)

914795 The infrared imaging spectrograph (IRIS) for TMT: overview of innovative science programs [9147-369]

S. A. Wright, Dunlap Institute for Astronomy and Astrophysics, Univ. of Toronto (Canada) and Univ. of Toronto (Canada); J. E. Larkin, Univ. of California, Los Angeles (United States); A. M. Moore, Caltech Optical Observatories (United States); T. Do, Dunlap Institute for Astronomy and Astrophysics, Univ. of Toronto (Canada); L. Simard, NRC - Herzberg Institute of Astrophysics (Canada); M. Adamkovics, Univ. of California, Berkeley (United States); L. Armus, California Institute of Technology (United States); A. J. Barth, E. Barton, Univ. of California, Irvine (United States); H. Boyce, Dunlap Institute for Astronomy and Astrophysics, Univ. of Toronto, (Canada) and Univ. of Saskatchewan (Canada); J. Cooke, Swinburne Univ. of Technology (Australia); P. Cote, T. Davidge, NRC - Herzberg Institute of Astrophysics (Canada); B. Ellerbroek, Thirty Meter Telescope Observatory Corp. (United States);

A. Ghez, Univ. of California, Los Angeles (United States); M. C. Liu, J. R. Lu, Institute for Astronomy, Univ. of Hawai'i (United States); B. A. Macintosh, Standford Univ. (United States); S. Mao, National Astronomical Observatories (China); C. Marois, NRC - Herzberg Institute of Astrophysics (Canada); M. Schoeck, Thirty Meter Telescope Observatory Corp. (United States); R. Suzuki, National Astronomical Observatory of Japan (Japan); J. C. Tan, Univ. of Florida (United States); T. Treu, Univ. of California, Santa Barbara (United States); L. Wang, Thirty Meter Telescope Observatory Corp. (United States); J. Weiss, Univ. of California, Los Angeles (United States)

9147 9T Drift scanning technique for mid-infrared background subtraction [9147-372] S. Heikamp, B. R. Brandl, C. U. Keller, Leiden Observatory (Netherlands); L. Venema, ASTRON (Netherlands); E. Pantin, CEA-Ctr. de SACLAY (France); R. Siebenmorgen, D. Ives, F. Kerber, European Southern Observatory (Germany)

Author Index 
Proc. of SPIE Vol. 9147 914701-62

Downloaded From: https://www.spiedigitallibrary.org/conference-proceedings-of-spie on 26 Apr 2023 Terms of Use: https://www.spiedigitallibrary.org/terms-of-use 


\title{
Conference Committee
}

\author{
Symposium Chairs
}

Gillian S. Wright, UK Astronomy Technology Centre (United Kingdom)

Luc Simard, National Research Council Canada (Canada)

Symposium Co-chairs

Colin Cunningham

UK Astronomy Technology Centre (United Kingdom)

Masanori lye

National Astronomical Observatory of Japan (Japan)

Conference Chairs

Suzanne K. Ramsay, European Southern Observatory (Germany)

Ian S. McLean, University of California, Los Angeles (United States)

Hideki Takami, Subaru Telescope, National Astronomical Observatory of Japan (United States)

Conference Program Committee

Julia J. Bryant, The University of Sydney (Australia)

Stephen S. Eikenberry, University of Florida (United States)

Chris J. Evans, UK Astronomy Technology Centre (United Kingdom)

Ramón J. García López, Instituto de Astrofísica de Canarias (Spain)

Maureen L. Savage, SOFIA / USRA (United States)

Luc Simard, National Research Council Canada (Canada)

Oskar von der Lühe, Kiepenhever-Institut für Sonnenphysik (Germany)

Session Chairs

1 Instrument Programs at Major Observatories

Hideki Takami, Subaru Telescope, National Astronomical Observatory of Japan (United States)

2 New Instruments and Upgrades to Existing Instruments

Oskar von der Lühe, Kiepenhever-Institut für Sonnenphysik (Germany)

3 High Multiplex and Survey Instruments I

Stephen S. Eikenberry, University of Florida (United States)

$4 \quad$ High Multiplex and Survey Instruments II

Ian S. McLean, University of California, Los Angeles (United States) 
$5 \quad$ High Multiplex and Survey Instruments III

Julia J. Bryant, The University of Sydney (Australia)

6 High Spectral Resolution Instruments I

Suzanne K. Ramsay, European Southern Observatory (Germany)

$7 \quad$ High Spectral Resolution Instruments II

Ramón J. García López, Instituto de Astrofísica de Canarias (Spain)

$8 \quad$ High Spatial Resolution Instruments I

Hideki Takami, Subaru Telescope, National Astronomical Observatory of Japan (United States)

$9 \quad$ High Spatial Resolution Instruments II

Maureen L. Savage, SOFIA / USRA (United States)

10 Instruments for Extremely Large Telescopes

Luc Simard, National Research Council Canada (Canada) 


\section{Introduction}

This conference, the fifth in the series on Ground-based and Airborne Instrumentation for Astronomy, attracted a very rich programme of papers discussing all aspects of the design and delivery of complete instrument systems. The range of innovation in the instrument building community was particularly remarkable at this meeting. Instrument concepts matched to the $\sim 1000 \mathrm{~m}^{2}$ collecting area of the extremely large telescopes were discussed alongside highly scientifically focused instruments for telescopes with $\sim 1 \mathrm{~m}^{2}$ apertures. Meanwhile, the current large telescope observatories are considering their instrument suites in the light of future new developments. Survey instruments are becoming an enduring and significant theme at our meeting, whether designed for observing hundreds of galaxies, or hundreds of points on a radial velocity curve to reveal exo-planetary systems.

At this meeting, $\sim 350$ papers were presented, either as oral or poster contributions. Six invited reviews were requested on major existing facilities or planned new ones. The presentations were arranged into 10 sessions over the five days of the meeting. The major topics were:

- Instrument Programmes at Major Observatories

- New instruments and Upgrades to Existing Instruments

- High Multiplex and Survey Instruments

- High Spectral Resolution Instruments

- High Spatial Resolution Instruments

- Instruments for Extremely Large Telescopes

On behalf of the program committee, it is a pleasure to acknowledge the contribution of all participants in making this a busy and very productive meeting.

\section{Suzanne K. Ramsay Hideki Takami Ian S. McLean}


Proc. of SPIE Vol. 9147 914701-66

Downloaded From: https://www.spiedigitallibrary.org/conference-proceedings-of-spie on 26 Apr 2023 Terms of Use: https://www.spiedigitallibrary.org/terms-of-use 